\title{
Standard Moment Method in the Problems on Ion Kinetics in Neutral Gas
}

\author{
A. Ya. Ender ${ }^{1, *}$, I.A. Ender ${ }^{2}$ and A.B. Gerasimenko ${ }^{1}$ \\ ${ }^{I}$ Ioffe Physical-Technical Institute of the Russian Academy of Sciences, Russia \\ ${ }^{2}$ St.-Petersburg State University, Russia
}

\begin{abstract}
A new method for calculating matrix elements of the collision integral is presented. This method is applied both for the determination of ion mobility on atom background and the construction of the ion distribution function in the case when an electric field or crossed electric and magnetic fields are switched on instantaneously. The limits of the nonstationary moment method are explored. Five interaction models are studied in detail, and the evolution of the distribution function is presented for various electric field strengths. It is shown that the angle and energy dependence of the crosssection strongly influences the distribution especially in the high velocity domain. In particular, the runaway of ions for the Coulomb interaction is determined. Moreover, the mobility vector is shown to move along a spiral to its stationary value when an electric and cross magnetic field are switched on.
\end{abstract}

Keywords: Boltzmann equation, distribution function, moment method, ion mobility, crossed electric and magnetic fields.

\section{INTRODUCTION}

The moment method advanced by Burnett [1] is one of the main methods for solving the Boltzmann equation. Nevertheless, there has been only a slow progress in the development of this method mainly because the matrix elements $(\mathrm{ME})$ of the collision integral remained to a large extend unknown especially in the nonlinear case. In [2-4] a number of relationships among the MEs has been uncovered. Recurrence procedures have been proposed in which all nonlinear MEs can be calculated in terms of the much simpler linear isotropic MEs. In the course of these calculations the linear MEs with large indices could be obtained, as well. Solving exemplarily the nonlinear relaxation of isotropic distribution functions (DFs), it was shown that the moment method not only can be used to build up the moments but also the DF itself, even for strong departures from equilibrium up to 5-10 times the thermal velocity $[2,3]$.

In the present work the moment method is applied to get the ion DF for both, an electric field and an electric and cross magnetic field, where use is made of an appropriate number of MEs. The motion of an admixture of charged particles in a quasi-neutral gas under the influence of external fields has been considered already half a century ago. Earliest kinetic approaches for solving the transport problem of charged particles can be found in the works of Sena [5], Wannier [6], Perel [7], and Smirnov [8]. General theoretical and experimental results, accomplished during the 70-80s of the last century, have been discussed in $[9,10]$. In these monographs a classification of problems is given also but it should be kept in mind that the majority of results has been obtained for two extreme cases only, namely very weak or very

*Address correspondence to this author at the Ioffe Physical-Technical Institute of the Russian Academy of Sciences, Russia; E-mail: andrei.ender@mail.ioffe.ru strong electric fields. A review of further works stemming from the last decades can be found in $[11,12]$.

Many papers on charged particle kinetics were initiated by Kumar [13], who advanced the original calculation of MEs using the Thalmi coefficients. A number of results have been obtained in [14], which has developed the methods for solving the Boltzmann equation in case of charged particles further. A cycle of works, which mainly developed such ideas in the theory of electron transport, was carried out by Robson, White, Ness and Li $[11,12,15,16]$.

Due to the complexity of the collision integral the approximated moment-transport theory (MTT) for solving problems of ion kinetics under an electric field has been widely used [17, 18]. It often achieves good accordance with experimental data concerning transport coefficients. Problems at the kinetic level have been generally attacked by the moment method by the use of polynomial expansions of the DF, namely by spherical Hermite polynomials (Burnett functions). In spite of the linearity of the collision integral much success could, however, not be made till now except for some models, which took into account a sufficient number of DF expansion terms.

The two-temperature method [10], replacing the standard one, places new hopes in overcoming these difficulties. Whereas in the standard method the temperature of the basic Maxwellian, about which the expansion is carried out, coincides with the neutral gas temperature, in the twotemperature (non-standard or modified) method these two temperatures are different from each other. Now, with linear MEs of large indices one can use the standard method in a new way. It should be emphasized that the application of MEs of large indices gives rise to ten thousands of DF moments whereas in the above mentioned works only about ten moments are involved. Since there are no further restrictions on the MEs, the limits in the application of the standard method can be investigated. In the following, we 
advance the non-stationary moment method for solving the Boltzmann equation. The central part of this method will be the solution of the ion evolution after a sharp switching on of an electric field at the moment of time $t=0$. As a result, not only the stationary state is found but also non-stationary features of the transient process. Note that the ion velocity $\mathrm{DF}$ at $t=0$ is the equilibrium (Maxwellian) distribution with the temperature equal to the atom temperature.

The structure of the present work, which includes two large parts, is as follows: in the first part the spatially uniform case of ion evolution under a sharp switching on of an electric field is treated, whereas the non-stationary process triggered by a sharp switching on of an electric and cross magnetic field is treated in the second part.

The first part (Sections 1.1 and 1.2) begins with various interaction models and the necessity of getting nonstationary solutions of the Boltzmann equation. Section 1.3 describes the solution method in detail. It includes the differential operator, corresponding to the left hand side of the Boltzmann equation, and the calculation of the matrix elements of the collision integral. In Section 1.4 the CEMmodel is under consideration. It refers to the ion resonance charge exchange process of atoms with cross-sections decreasing inversely proportional to the relative velocity. For this model the analytic solution for the DF is built up together with the moments for the non-stationary problem. With the help of the analytical solution a convergence study of the moment method is made. It is shown that there are insurmountable difficulties in building up the stationary solution via the stationary method even in the domain of moderate electric fields. In Section 1.5 the non-stationary problem is solved for several interaction models. It is shown that the interaction model strongly influences the physical moments and the behavior of the DF especially in the high velocity region. Under Coulomb interaction and not so weak electric fields the phenomenon of ion runaway is investigated. In Section 1.6 two methods to improve convergence are considered. It is shown that the transition to the two-temperature method results in a better convergence. The new matrix elements, which arise by the transition to the non-standard method, can be built up with the known nonlinear MEs from the standard moment method. An essential improvement in the convergence can be achieved if only spherical harmonics are involved in the expansion of the DF and not Sonine polynomials. For the hard-sphere model, the kernels of the corresponding integral operators have been constructed in $[19,20]$. Using these kernels, we extend the study of the DF into the domain of high velocities in the case of a weak electric field. With this method the DF can be calculated up to arbitrarily high velocities and, hence, stationary DFs can be found.

The second part (Section 2.1) begins with the construction of the system of moment equations describing the ion kinetics in an electric and cross magnetic field. It is shown that for models with diagonal interaction matrix an exact analytic solution can be obtained. A nice coincidence between analytical and numerical solutions is shown for pseudo-Maxwellian molecules. In Section 2.2 the notion of reduced physical moments is introduced, being calculated in Section 2.3. Particular emphasis is laid on their behavior for very weak electric fields, i.e. on the area where the Chapman-Enskog method is applicable. It is shown that with increasing magnetic field the moments approach nonmonotonously the stationary value, passing an oscillatory stage with the ion Larmor frequency as the main frequency. With an increase of the electric field the peculiarities of the non-stationary process pertain. The runaway effect is diminished by the magnetic field in case of Coulomb interaction. For very strong magnetic fields the convergence of the moment method is restored at large time values and very strong electric fields. In Section 2.4 the ion distribution function is calculated for an electric and cross magnetic field for different interaction models. Interesting features of the DF are found in the domain of high velocities for the Coulomb interaction.

\section{Electric Field Switch-On}

\subsection{Statement of the Problem}

Consider a small ion admixture in a gas with Maxwellian velocity distribution at fixed temperature. The electric field $\mathbf{E}$ switch-on results in arising the ion flow along the field direction.

Kinetic theory of a neutral gas is well-developed at the weak deviations from equilibrium. Such is the ChapmanEnskog method as well as calculation of the transport coefficients for neutral gases $[21,22]$. This method involves the linearization of Boltzmann equation with a small correction of DF to the equilibrium (Maxwellian) distribution.

Under electric field, transferring to the linear equation for a small admixture of charged particles is easier as compared with a neutral gas. Here, two eventual sources of nonlinearity exist: the direct (due to ion-ion interactions) and non-direct (due to possible distortion of atomic distribution by ions and its reverse effect on distribution of the ions). Both sources are sufficiently small if ion density is very small versus background gas density.

For the case under consideration, the general transport processes are diffusion and mobility. A relation of ion flow $\mathbf{J}$ with density gradient $n$ at a small quantity of $\nabla n$ is governed by the Fick's law

$\mathrm{J}=-D \nabla n$.

Here, $D$ is the diffusion coefficient.

If the electric field is applied to spatially uniform mixture, an ion flow also arises. The velocity of mass center of ion cloud (mean ion velocity) is called a drift velocity $\mathbf{v}_{d}$. If the field is sufficiently weak, the drift velocity is directly proportional to the field strength:

$\mathbf{v}_{d}=K \mathbf{E}$.

For the case of a weak external field, the mobility $K$ and diffusion coefficient $D$ are related by Einstein relationship

$K=\frac{e D}{k T}$,

where $e$ is ion charge, $k$ is Boltzmann constant, $T$ is gas temperature. 
If the strength is increased in such a manner that a mean energy of ions is commensurable (or even larger) with thermal energy of atoms, the situation will change. First, a mobility $K$ in (2), strictly speaking, is not constant but depends on $E$. Nevertheless, it is of use to involve (2) for determination of $K$ at any electric field. Second, the diffusion coefficient becomes a tensor. Signify, that when calculating diffusion of ions even at the strong fields, it is assumed that the density gradient is of a small value. Note, that a study of diffusion is out of scope of the present paper.

The very fact that, for ion admixture in a neutral gas, the collision integral is a linear operator, does not assume a smallness of deviation from equilibrium. Moreover, one can assert that an extent of deviation from equilibrium turns out to be extremely high under the strong electric field.

The strongly non-equilibrium process is described by the Boltzmann equation which, in the case under consideration, is

$$
\frac{\partial f}{\partial t}+v \cdot \frac{\partial f}{\partial \mathbf{r}}+\frac{e \mathbf{E}}{m_{i}} \cdot \frac{\partial f}{\partial \mathbf{v}}=J(f) .
$$

Here, $f(\mathbf{v}, \mathbf{r}, t)$ is the DF of ions, $\mathbf{E}$ is the electric field strength, $J(f)$ is the linear collision integral of ions with the Maxwellian distribution of atoms, $m_{i}$ is ion mass. The Boltzmann equation in the spatially uniform case is

$$
\frac{\partial f}{\partial t}+\frac{e \mathbf{E}}{m_{i}} \cdot \frac{\partial f}{\partial \mathbf{v}}=J(f) .
$$

As a rule, a steady state, which occurs due to a balance of ion acceleration between its collisions with atoms and its deceleration during collision process, is under consideration in literature. The steady-state distribution function $f_{s t}$ satisfies the stationary Boltzmann equation

$$
\frac{e \mathbf{E}}{m_{i}} \cdot \frac{\partial f_{s t}}{\partial \mathbf{v}}=J\left(f_{s t}\right)
$$

Involving the stationary DF, the stationary drift velocity and mobility are found. If one needs a diffusion tensor, it can be built up only knowing $f_{s t}(\mathbf{v})$. As far as under strong electric field due to the gradient the DF deviation occures not from the Maxwellian distribution but from the strongly nonequilibrium stationary DF. Thus, the building-up of (6) solution is the necessary condition for obtaining all the transport coefficients for the ions in a neutral gas and, hence, presence of the field term results in essential distinctions from the Chapman-Enskog method concerning a neutral gas.

The question now arises of whether there is always a solution of equation (6). This point was discussed in [14]. If for rather hard potentials (e.g., for hard-sphere model) the theorem of solution existence and uniqueness is approved for equation (6), then for Coulomb interaction it is far from being evident. For Coulomb interaction, the runaway effect for ions can take place, when a portion of ions consuming large energy from the field, subsequently are accelerated infinitely.

An answer on a question of existence of the steady state can be obtained when building up the solution of the nonstationary equation (5). Let at first the ions are in equilibrium with atoms, and for a time $t=0$ the electric field $\mathrm{E}$ is switched on. Then a transient process arises. If at large time the constant DF maintains, it will be a solution of the stationary Boltzmann equation (6). On the contrary, if the process is not finished yielding the steady state, then, solving the non-stationary equation, the outgoing ion flow formation can be followed up. The DF building-up for transient stage is of primary interest, especially, if can be followed up a change in the features of this process when changing the ionatom scattering cross-section. Generally, the steady-state transport coefficients are under investigation in literature. We have proposed more intricate problem, namely, to study a DF behaviour in the non-stationary process when the electric field is involved. Our main problem, i.e., the study of application limits of the moment method, is being solved simultaneously.

Let the ion mass $m_{i}$ is equal to that of an atom $m_{a}$. Note, that this case is more difficult one, because the collision integral is essentially simpler in the limiting cases $m_{i} / m_{a} \rightarrow 0$ and $m_{i} / m_{a} \rightarrow \infty$. Besides, assuming $m_{i}=m_{a}$, the processes of the resonant charge exchange of the ions on atoms, inherent for ion kinetic in their own gas can be considered.

\subsection{Models of Interaction}

The list of interaction models and their descriptions involved in calculation is shown below.

For power potentials when an interaction potential $V$ depends on a distance $r$ as a power function $\left(V \sim \frac{1}{r^{v}}\right)$, a scattering cross-section is presented as follows

$\sigma(g, z)=g^{\gamma-1} F(z), \quad z=\sin ^{2}(\theta / 2), \quad \gamma=(v-4) / v$.

Here, $g$ is a value of relative velocity, $\theta$ is a scattering angle, and $F(z)$ is the angular part of scattering cross-section which is unequivocally determined by a parameter $v$. Remember that for the hard-sphere model the scattering is isotropic, i.e., $F(z)=$ const. The model of pseudo-power potential is often used in kinetics. In such model the scattering by angles is assumed to be isotropic, and dependence of cross-section on velocity module is the same as for power potential.

A set of models of "quasi-power potentials" can be determined with a fixed value of $v$ (or $\gamma)$ for which is conserved a dependence on $g$ for power potential and the angular dependence is supposed to be arbitrary. The temperature dependence of $\Omega$-integrals is the same for all the models of quasi-power potentials with a fixed $v$. Hence, all MEs are also equally dependent on a temperature, namely, $\sim T^{\mu}$, where $\mu=\gamma / 2=(v-4) /(2 v)$. E.g., $\mu=0$ for the Maxwellian molecules, $\mu=0.5$ for hard spheres, $\mu=-1.5$ for the case of Coulomb interaction.

In ion kinetics, under interaction with atoms of the same kind (in their own gas), a resonant charge exchange of ions on atoms plays an essential role. This phenomenon occurs during electron exchange: ion flows past atom and obtains an electron, the atom is conversed into ion and the ion into atom. This phenomenon occurs at the collisions with a large impact parameter and practically has no effect on a direction of the heavy particle motion, i.e. the angular part of cross- 
section is equivalent to $180^{\circ}$ scattering in the case of resonant charge exchange.

Consider several models of interaction:

1. Pseudo-maxwellian molecules, the total cross-section is inversely proportional to relative velocity $(\Sigma=$ $\mathrm{C} / \mathrm{g}$ ), the angular part of cross-section is isotropic.

2. The CEM-model, the total cross-section is also inversely proportional to the relative velocity, the angular part of cross-section - resonant charge exchange - corresponds to $180^{\circ}$ scattering.

Collision frequency does not depend on velocity, for these two models. So, they are called the models " $\tau=$ const".

3. The HS-model - hard-spheres model, the total crosssection does not depend on velocity $(\Sigma=$ const $)$, angular part of cross-section corresponds to isotropic scattering.

4. The CEHS-model, the total cross-section does not depend on velocity $(\Sigma=$ const $)$, the angular part of cross-section corresponds to resonant charge exchange.

A mean free path does not depend on velocity for HS and CEHS models. So, they are called the models " $\lambda$ = const".

5. Coulomb interaction, $V \sim 1 / r$. Scattering cross-section is determined via Rutherford formula.

A rapid decrease in a scattering cross-section with an increase in a relative velocity and divergence at small scattering angles is inherent for this model. To remove this divergence, screened Coulomb potential with a screening radius equal to Debye radius $\lambda_{D}$ is used:

$$
\sigma(g, \theta)=\frac{e^{4}}{m^{2} g^{4} \sin ^{4} \frac{\theta}{2}}
$$

Here, $n$ is plasma density. As a result, the collision integral turns out to be proportional to the Coulomb logarithm

$$
\ln \Lambda=\ln \left(12 \pi n \lambda_{D}^{3}\right) .
$$

In our calculation the Coulomb logarithm is assigned to be 5. When calculating the MEs in the standard normalization, in the case of the Coulomb interaction, the time unit is assigned to be

$$
r=16(k T / m)^{3 / 2} /\left(J_{1} n\right), \quad J_{1}=8 \pi e^{4} \ln \Lambda / m^{2} .
$$

\subsection{Method of Solution}

We use the moment method in solving the Boltzmann equation. Perform the DF expansions in terms of the spherical Hermite polynomials (Burnett functions) [1], [3]

$$
\begin{aligned}
& f(\mathbf{c}, \mathbf{r}, t)=M(\alpha, c) \sum C_{r l m}^{i}(\mathbf{r}, t) H_{r l m}^{i}(\mathbf{c}), \quad \alpha \frac{m_{i}}{2 K T}, \\
& H_{j}=Y_{l m}^{i}(\Theta, \varphi) c^{l} S_{l+1 / 2}^{(r)}\left(c^{2}\right), \quad i=0,1 ; \quad \mathbf{c}=\sqrt{\alpha}(\mathbf{v}-\mathbf{u}),
\end{aligned}
$$

$Y_{l m}^{0}(\Theta, \varphi)=P_{l}^{m}(\cos \Theta) \cos m \varphi, \quad Y_{l m}^{1}(\Theta, \varphi)=P_{l}^{m}(\cos \Theta) \sin m \varphi$, $0 \leq m \leq l$.

Here, an index $j$ includes four indices $(r, l, m, i), M$ is the weight Maxwellian of a temperature $T$ and mean velocity $\mathbf{u}, Y_{l m}^{i}(\theta, \phi)$ are the real spherical harmonics, $P_{l}^{m}(\cos \Theta)$ are the associated Legendre polynomials, and $S_{l+1 / 2}^{(r)}\left(c^{2}\right)$ are the Sonine (Laguerre) polynomials. The distribution function should satisfy a condition

$\int_{0}^{\infty} f^{2} \exp \left(c^{2}\right) d^{3} c<\infty$,

for (10) convergence. We call (13) the Grad criterion. The constraint, related with the Grad criterion, arises when expanding in terms of the Sonine polynomials but not the spherical harmonics as the Sonine polynomials do to be orthogonal with Maxwellian weight.

In the most general case, a behaviour of gas mixture is described by a system of non-linear Boltzmann equations. In order to avoid extra indices and cumbersome calculations let's write out the non-linear Boltzmann equation for onecomponent gas:

$\frac{\partial f}{\partial t}+\mathbf{v} \cdot \frac{\partial f}{\partial \mathbf{r}}+\frac{\mathbf{F}}{m} \cdot \frac{\partial f}{\partial \mathbf{v}}=\hat{I}(f, f)$.

Here, $\hat{I}(f, f)$ is the non-linear collision integral, $F$ is a vector of external force, $m$ is a particle mass. In the moment method, an equation (14) is replaced by the infinite system of moment equations:

$\frac{D\left(C_{i}\right)}{D t}=\sum_{j, k} K_{j, k}^{i} C_{j} C_{k}$.

An operator $D / D t$ in (15) refers to the differential (left) side of the Boltzmann equation. It was studied in [1] in the absense of external forces.

\subsubsection{Matrix Elements of the Collision Integral}

Non-linear matrix elements $K_{j, k}^{i}$ are determined as follows:

$K_{j, k}^{i}=\int H_{i} I\left(M H_{j}, M H_{k}\right) d^{3} v / g_{i}, \quad g_{i}=\int M H_{i}^{2} d^{3} v$.

The linear MEs correspond to the linear collision integral and can be of the first $\left(K_{j, 0}^{i}\right)$ and second $\left(K_{0, k}^{i}\right)$ kinds. They are denoted by $\Lambda$. In the axially symmetric case $(m=0)$ we have

$\Lambda_{r, r_{1}, l}^{(1)}=K_{r_{1}, l, 0,0}^{r, l} ; \quad \Lambda_{r, r_{1}, l}^{(2)}=K_{0,0, r_{2}, l}^{r, l}$.

When calculating a small ion admixture on atom background, the linear matrix elements of the first kind are needed.

In $1 D$-spatial problems, the velocity $\mathrm{DF}$ is axiallysymmetric and may be expanded by the spherical Hermite polynomials with two indices $r$ and $l: H_{r l}=S_{l+1 / 2}^{r} P_{l}$. It was shown in [3] that $3 D$ matrix elements are proportional to the corresponding axially symmetric MEs and the coefficients of 
proportionality are easily expressed via the Klebsh-Gordon coefficients.

The invariance of the collision integral relative to a choice of a basis functions set, in terms of which the DF expansion is carried out $[3,4]$ is laid as a base in obtaining the relationships between the MEs. The bases considered are distinct in the parameters of the weight Maxwellian. In the axially symmetric case those are a temperature $T$ and a mean velocity value $u$, which are denoted by unique letter $W$. The expansion coefficients of the DF in the bases $W_{0}$ and $W_{1}$ are interrelated via transport matrix $D$ :

$C_{j}^{1}=\sum_{k=0}^{\infty} D_{j, k}\left(W_{1}, W_{0}\right) C_{k}^{0}$

Amatrix $D$ was built up in [3] using $\alpha-u$-representation of the Boltzmann equation [23]. It follows from the invariance principle of the collision integral relative to a choice in the basis functions that the MEs of the collision integral in two different bases are mutually expressed in this way

$K_{k^{\prime}, j^{\prime}}^{i^{\prime}}\left(W_{1}\right)=\sum_{i} D_{i^{\prime}, i}\left(W_{1}, W_{0}\right) \sum_{k, j} K_{k, j}^{i}\left(W_{0}\right) D_{k, k^{\prime}}\left(W_{0}, W_{1}\right) D_{j, j^{\prime}}\left(W_{0}, W_{1}\right)$.

If this expression is differentiated with respect to $T_{1}$ or $u_{1}$ and set $W_{1}=W_{0}\left(u_{1}=u_{0}, T_{1}=T_{0}\right)$, we obtain the "temperature" recurrence relationships

$\left(T \frac{d}{d T}-R\right) K\left(\begin{array}{c}a \\ a, b\end{array}\right)_{r_{1}, l_{1}, r_{2}, l_{2}}^{r, l}=r K\left(\begin{array}{c}a \\ a, b\end{array}\right)_{r_{1}, l_{1}, r_{2}, l_{2}}^{r-1, l}$

$-\left(r_{1}+1\right) K\left(\begin{array}{c}a \\ a, b\end{array}\right)_{r_{1}+1, l_{1}, r_{2}, l_{2}}^{r, l}-\left(r_{2}+1\right) K\left(\begin{array}{c}a \\ a, b\end{array}\right)_{r_{1}, l_{1}, r_{2}+1, l_{2}}^{r, l}$,

where $R=r_{1}+r_{2}-r+\left(l_{1}+l_{2}-l\right) / 2$ and the "velocity" recurrence relationships

$$
\begin{array}{r}
\beta(l-1) K\left(\begin{array}{c}
a \\
a, b
\end{array}\right)_{r_{1}, l_{1}, r_{2}, l_{2}}^{r, l-1}+\gamma(r-1, l+1) K\left(\begin{array}{c}
a \\
a, b
\end{array}\right)_{r_{1}, l_{1}, r_{2}, l_{2}}^{r-1, l+1} \\
-\beta\left(l_{1}\right) K\left(\begin{array}{c}
a \\
a, b
\end{array}\right)_{r_{1}, l_{1}+1, r_{2}, l_{2}}^{r, l_{1}}-\gamma\left(r_{1}, l_{1}\right) K\left(\begin{array}{c}
a \\
a, b
\end{array}\right)_{r_{1}+1, l_{1}-1, r_{2}, l_{2}}^{r, l} \\
-\sqrt{\left(m_{b} / m_{a}\right)}\left(\beta\left(l_{2}\right) K\left(\begin{array}{c}
a \\
a, b
\end{array}\right)_{r_{1}, l_{1}, r_{2}, l_{2}+1}^{r, l}\right. \\
\left.-\gamma\left(r_{2}, l_{2}\right) K\left(\begin{array}{c}
a \\
a, b
\end{array}\right)_{r_{1}, l_{1}, r_{2}+1, l_{2}-1}^{r, l}\right)=0, \\
\beta(l)=-\frac{l+1}{2 l+1}, \quad \gamma(r, l)=\frac{(r+1) l}{2 l+1} .
\end{array}
$$

Here, the matrix elements $K\left(\begin{array}{c}a, b \\ a, b\end{array}\right)_{r_{1}, l_{1}, r_{2}, l_{2}}^{r, l}$ correspond to a mixture of two kinds of the particles $a$ and $b$ with masses $m_{a}$ and $m_{\mathrm{b}}$, the particles of $a$ kind being scattered on the particles of $b$ kind. A relationship (20) becomes the simplest for the power potentials (7), when the MEs are proportional to $T^{\mu}$. In this case, a left side of (20) takes on a form

$\left(T \frac{d}{d T}-R\right) K\left(\begin{array}{c}a \\ a, b\end{array}\right)_{r_{1}, l_{1}, r_{2}, l_{2}}^{r, l}=(\mu-R) K\left(\begin{array}{c}a \\ a, b\end{array}\right)_{r_{1}, l_{1}, r_{2}, l_{2}}^{r, l}$

For arbitrary potentials, in [3], an independent on interaction cross-section, universal recurrence formulas for the expansion coefficients of the non-linear MEs are deduced in terms of $\Omega$-integrals well-known in kinetic theory. A simple relation for $\Omega$-integrals, which includes the derivative with respect to temperature, turns out to be very important in derivation of these relationships.
Note, that the recurrence relationships (20) and (21) are valid also for the MEs of the gain and loss terms separately.

The recurrence procedures were developed. It was shown that all non-linear (and linear) MEs can be found, if the linear isotropic $(l=0)$ MEs of one kind are known. Simple formulas [2] and [3] are obtained for linear isotropic MEs of the first kind as well as of the second kind. The software package for calculation of all MEs up to those of very large indices for arbitrary masses of colliding particles was created.

It should be noted, that the linear MEs with $l \neq 0$ are found using only a full recurrence procedure for nonlinear MEs. So, when building-up any (even the linear ones) MEs, the total collision integral in the Boltzmann equation should be considered.

\subsubsection{System of Moment Equations}

In the presence of external forces, the left side of moment equations (15) was deduced by us in [24] both in global and in arbitrary local bases. Here, we present only a final form of the moment system in the global basis

$$
\frac{\partial C_{J}}{\partial t}+\mathbf{u} \cdot \frac{\partial C_{J}}{\partial \mathbf{r}}+\frac{1}{\alpha^{1 / 2}} \sum_{k=1}^{3} \frac{\partial}{\partial x_{k}} G_{j}^{(k)}+\alpha^{1 / 2} \sum_{k=1}^{3} \frac{F_{k}}{m_{0}} L_{j}^{(k)}=\sum_{j_{1}, k} K_{j_{1}, k}^{j} C_{j i} C_{k} .
$$

Remember that

$$
C_{j}(\mathbf{r}, t)=\frac{1}{g_{j}} \int f(\mathbf{v}) H_{j}(\mathbf{c}) d^{3} v \text {. }
$$

A normalization factor $g_{j}$ is as follows [3]

$g_{j}=y_{l m} \sigma_{r l}, \quad y_{l m}=\frac{2 \pi\left(1+\delta_{m 0}\right)(l+m) !}{(l-m) !(2 l+1)}, \quad \sigma_{r l}=\frac{\Gamma(r+l+3 / 2)}{2 \pi^{3 / 2} r !}$

For $G_{j}^{(k)}$ and $L_{j}^{(k)}$, the expressions are obtained as stated below

$$
\begin{aligned}
& G_{j}^{(1)}=-\frac{1}{2(2 l+3)}\left((l+r+3 / 2) d_{m-1} C_{r l+1 m-1}^{i}-r d_{m-1} C_{r-1 l+1 m-1}^{i}\right. \\
& \left.-(l+m+2)(l+m+1)\left((l+r+3 / 2) C_{r l+1 m+1}^{i}-r C_{r-1 l+1 m+1}^{i}\right)\right) \\
& +\frac{1}{2(2 l-1)}\left(d_{m-1}\left(C_{r l-1 m-1}^{i}-C_{r+1 l-1 m-1}^{i}\right)\right. \\
& -(l-m)(l-m-1)\left(C_{r l-1 m+1}^{i}-C_{r+1 l-1 m+1}^{i}\right) \text {, } \\
& G_{j}^{(2)}=-\frac{(-1)^{i+1}}{2(2 l+3)}\left((l+r+3 / 2) d_{m-1} C_{r l+1 m-1}^{1-i}-r d_{m-1} C_{r-1 l+1 m-1}^{1-i}\right. \\
& \left.+(l+m+2)(l+m+1)\left((l+r+3 / 2) C_{r l+1 m+1}^{1-i}-r C_{r-1 l+1 m+1}^{1-i}\right)\right) \\
& +\frac{(-1)^{i+1}}{2(2 l-1)}\left(d_{m-1}\left(C_{r l-1 m-1}^{1-i}-C_{r+1 l-1 m-1}^{1-i}\right)\right. \\
& +(l-m)(l-m-1)\left(C_{r l-1 m+1}^{1-i}-C_{r+1 l-1 m+1}^{1-i}\right), \\
& G_{j}^{(3)}=\frac{l+m+1}{2 l+3}\left((l+r+3 / 2) C_{r l+1 m}^{i}-r C_{r-1 l+1 m}^{i}\right) \\
& +\frac{l-m}{2 l-1}\left(C_{r l-1 m}^{i}-C_{r+1 l-1 m}^{i}\right), \\
& L_{j}^{(1)}=\frac{1}{2 l-1}\left((l-m)(l-m-1) C_{r l-1 m+1}^{i}-C_{r l-1 m-1}^{i}\right) \\
& -\frac{r}{2 l+3}\left((l+m+1)(l+m+2) C_{r-1 l+1 m+1}^{i}-C_{r-1 l+1 m-1}^{i}\right),
\end{aligned}
$$




$$
\begin{aligned}
L_{j}^{(2)} & =(-1)^{i+1} \frac{1}{2 l-1}\left((l-m)(l-m-1) C_{r l-1 m+1}^{1-i}+C_{r l-1 m-1}^{1-i}\right) \\
& +\frac{r}{2 l+3}\left((l+m+1)(l+m+2) C_{r-1 l+1 m+1}^{1-i}+C_{r-1 l+1 m-1}^{1-i}\right), \\
L_{j}^{(3)} & =\frac{2}{2 l+3} r(l+m+1) C_{r-1 l+1 m}^{i}-\frac{2}{2 l-1}(l-m) C_{r l-1 m}^{i} .
\end{aligned}
$$

Here, has been used a denomination $d_{m}=1+\delta_{m 0}$.

\subsubsection{System of Moment Equations for Ion Admixture}

For the ion DF $f$ in the dimensionless form, the Boltzmann equation (5) is as follows

$$
\frac{\partial f}{\partial t}+\in \frac{\partial f}{\partial c_{z}}=\hat{J}(f)
$$

Here, thermal velocity $v_{T}=\sqrt{2 k T / m_{i}}$ is taken as velocity unit. Definitions mentioned above remain for the dimensionless DF and time: $f(\mathrm{c}, t) . \hat{J}(f)$ is the dimensionless collision integral, $\in$ is the dimensionless electric field strength. The direction of $z$-axis coincides with that of the electric field in equation (32). Time between collisions $\tau$ is taken as a time unit. The selection of time unit is evident for the models with $\tau=$ const. Transferring to these units, we obtain for the dimensionless electric field

$\in=\frac{e E \tau}{m_{i} v_{T}} \equiv \frac{e E \tau}{\sqrt{2 k T m_{i}}}$

However, for other interaction cross-sections, collision frequency depends on relative velocity, and selection of time unit is not evident. E.g., for the hard-sphere model, scattering cross section does not depend on velocity. Thus, the collision frequency turns out not to be constant but a mean free path $\lambda=\left(N_{a} \pi d^{2}\right)^{-1}$, where $N_{a}$ is density of atoms and $d$ is a diameter (in general case, a sum of colliding particles' radii). For the models with $\lambda=$ const, naturally, $\lambda$ can be taken as length unit.

With these units of length and velocity the time $\tau$ is determined unequivocally as $\tau=\lambda / v_{T}$. Then, for $\varepsilon$ we have

$\in=\frac{e E \lambda}{2 k T}$

The moment method in which the expansion of distribution function is carried out about the Maxwellian with atom temperature we will call the standard moment method. In this paper we generally demonstrate capabilities of the standard moment method when a large set of MEs is known.

The system of dimensionless moment equations corresponding to the Boltzmann equation (32), takes a form

$$
\frac{\partial C_{r, l}}{\partial t}+\in\left(\frac{2}{2 l+3} r(l+1) C_{r-1, l+1}-\frac{2 l}{2 l-1} C_{r, l-1}\right)=\sum_{r_{1}} \Lambda_{r, r_{1}, l} C_{r_{1}, l}
$$

It is easy to verify by substituting an expression (31) into general moment system (23), taking into consideration no spatial derivatives and symmetry of the problem relative to $z$-axis $(m=0)$. If the terms involving time derivatives are omitted in the moment equations (35) and the distinctions in determination of collision frequency as well as in normalization choice are taken into consideration, the system coincides totally with the stationary system in [9], for which solution Kihara [25] advanced the iteration method.

As a rule, when solving the kinetic equations for ions at the external field, one seeks the stationary state of the system. We advance the non-stationary approach, reserving the terms with time derivative in the system of moment equations. When solving the non-stationary moment system, the 4 th-order Runge-Kutta method is used.

\subsection{CEM-Model}

When studying the moment method convergence, it is of importance to have the analytical solutions of the problem if only for particular cases. The very simple model is CEMmodel. For this model, a collision operator takes a form

$J=\frac{M(c)-f(\mathbf{c})}{\tau}$.

It apparently coincides with BGK-model [26], which is often used to obtain the analytical and calculated solutions in a gas of a single component. If BGK-model is aimed to simplify the collision integral and has no physical foundation, CEM-model arises from a choice of the well determined scattering cross-section. At the same time, CEMmodel has all advantages of BGK-model and can be used to build up analytical solutions.

\subsubsection{Analytical Solutions}

It should be noted that CEM-model attracts a number of authors [14, 27, 28] due to its simplicity in the transport theory of charged particles. However, the DF was built up only for a cold gas or for the stationary case. Our purpose is to build up the non-stationary analytical solution for arbitrary temperature and to explore the capabilities of the nonstationary moment method by the example of the nonstationary analytical solution. For this model the matrix elements $\Lambda_{r, r_{1}, l}$, in the moment system (35) take a form

$\Lambda_{r, r_{1}, l}=\left(\delta_{l 0} \delta_{r 0}-1\right) \delta_{r r_{1}}$.

One can see that the ME matrix is diagonal at any $l$ and its eigen values, besides $\Lambda_{0,0,0}$, are equal to -1 . The system (35) for such interaction matrix is recurrently solvable and, at $r=0$ and $l=1$, we have

$C_{01}=2 \in(1-\exp (-t))$.

A drift velocity of ions is $u_{d}=C_{01} / 2$. Thus, the mobility in taken units at any electric field for CEM-model is

$K=1-\exp (-t)$.

The Boltzmann equation for CEM-model takes form

$\frac{\partial f}{\partial t}+\in \frac{\partial f}{\partial c_{z}}+f=M$.

The derivative with respect to $t$ is omitted in (40) in the stationary case. One can show, primarily, solving the uniform equation, that equation (40) has the following solution in the stationary case 

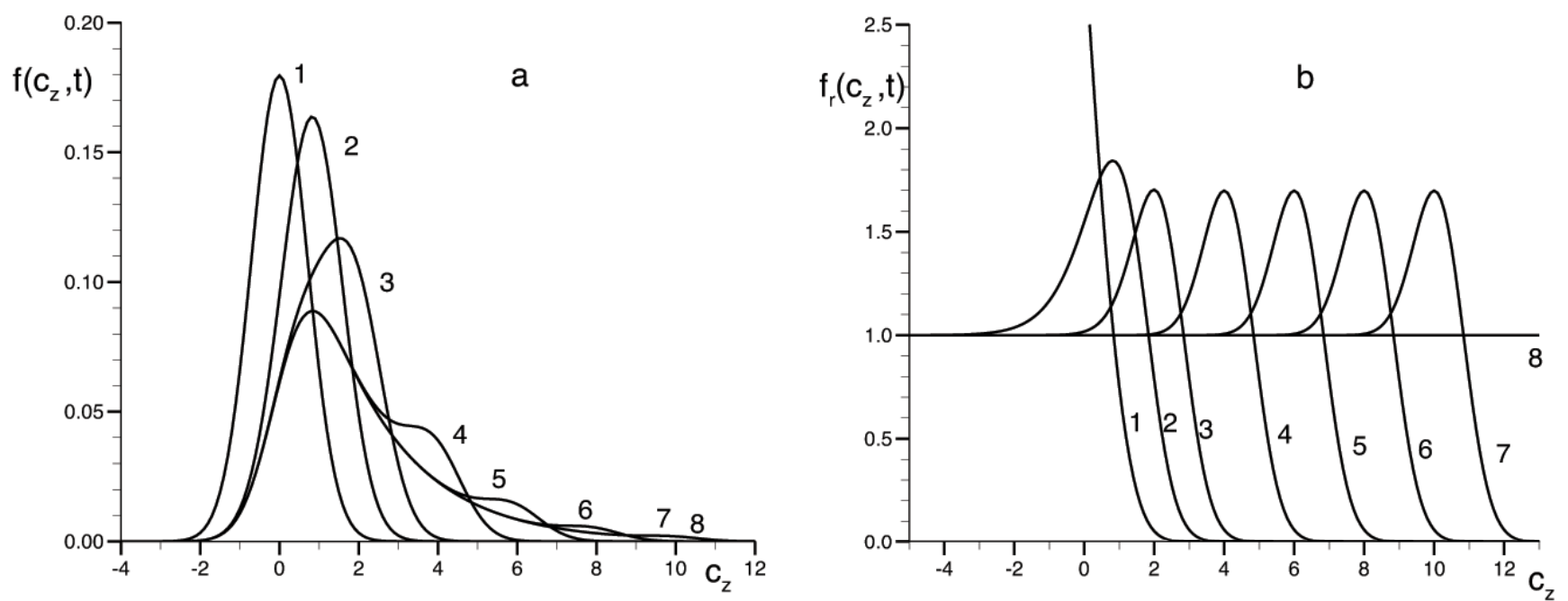

Fig. (1). Distribution functions at axis of symmetry at different times at $\in=2: f\left(c_{z}, t\right)(\mathbf{a})$ and $f_{r}\left(c_{z}, t\right)=f\left(c_{z}, t\right) / f\left(c_{z}, \infty\right)(\mathbf{b})$. Analytical solution. CEM-model. $t=0$ for Curve 1, 0.5 (2), 1 (3), 2 (4), 3 (5), 4 (6), 5 (7), 10 (8).

$f\left(c_{\rho}, c_{z}\right)=M\left(c_{\rho}\right) f\left(c_{z}\right)$

$f\left(c_{z}\right)=\frac{\sqrt{\pi}}{2} M\left(c_{z}\right) \frac{1}{\in} \exp \left(Z^{2}\right)(1+\operatorname{erf}(Z))$,

$Z=c_{z}-\frac{1}{2 \in}$.

Consider a non-stationary process when the electric field is switched-on instantaneously. In this case, the field is 0 at $t<0$ and is a constant value $\in$ at $t \geq 0$. At taken initial conditions, the DF for transverse component $\left(c_{\rho}\right)$ remains always the Maxwellian one. Divide both parts of (40) by $M\left(c_{\rho}\right)$ and retain a notion $f$ for the rest of DF. To solve the equation obtained, we use the method of characteristics and transfer from the variables $t, c_{z}$ to the variables $t, Y=c_{z}-\in t$. After certain transformations (see details in [29]), we obtain

$$
\begin{aligned}
& f\left(c_{z}, t\right)=\frac{\sqrt{\pi}}{2} M\left(c_{z}\right) e^{Z^{2}} \frac{1}{\in}(\operatorname{erf}(\varepsilon t-Z)+\operatorname{erf}(Z)) \\
& +e^{-t} M\left(c_{z}-\in t\right) .
\end{aligned}
$$

At $t \rightarrow \infty$ the DF goes toward the stationary solution built up above (41). Note, that for CEM-model the solution can be obtained for other non-stationary processes as well. E.g., to describe ion behaviour in the periodical external electric field. Here also the method of characteristics can be used.

In Fig. (1) the calculation results for DF are shown via analytical formulas (42) at $\epsilon=2$. One can see the spreading of the front in a velocity space along the electric field, which is followed by stationary DF. It is seen in Fig. (1) that relaxation in a domain of positive values of $c_{z}$ proceeds very slowly and the stationary solution output occurs with a large delay in this velocity range. When studying the moment method convergence, it is of principle the Grad criterion to be held, for which a rapid decrease of the DF at infinity is necessary. It is shown that the stationary solution for the interaction model under consideration does not satisfy the Grad criterion. However, the DF in a domain of high velocities during a non-stationary process at any finite time $t$ decreases so fast that the Grad criterion holds, and there are no crucial constraints on the moment method convergence. As a result, during a non-stationary process a larger part of the stationary DF can be built-up.

Besides the analytical solution directly for the DF in the case of CEM-model, we gain the analytical solution of the moment system (35). Starting from $C_{00}=1$, we obtain easily a solution for a moment $C_{01}(38)$. Next, we find the solutions in the layers $N=l+2 r=$ const, consequently increasing $N$. It can be shown that the analytical solution of a system (35) takes a form

$C_{r, l}(t)=B_{r, l} \in{ }^{N} S_{N}(t), \quad N=l+2 r$,

where a coefficient $B_{r, l}$ equals

$B_{r, l}=(-1)^{r} \frac{(2 l+4 r) ! !(2 l+1)}{2^{r}(2 r+2 l+1) ! !}$.

A function $S_{N}(t)$ is the same for a whole layer $N=1+2 r$ :

$S_{N}(t)=1-\exp (-t) \sum_{k=0}^{N-1} \frac{t^{k}}{k !}$.

This function turns out to be zero at $t=0$ and unity at $t$ $\rightarrow \infty$. However, a time delay for the saturation attainment increases with an increase in $N$.

\subsubsection{Study on the Moment Method Convergence by CEM- Model Example}

For CEM-model, one can compare always the DF restored via its moments with the exact solution and assert objectively the moment method convergence. Conclusions for CEM-model turn out to be true also on numerous occasions, for other interaction potentials. Also, question of convergence of DF restored via its moments built up analytically can be separated from the study of the calculation errors in the very moment system solution.

When solving numerically the kinetic problems by the moment method, the cut-off of the system of moment equations occurs inevitably a finite domain which contains the indices $l$ and $r$, being preferred. We study in what manner a shape of this domain affects the convergence. 
Performing a cut-off, it is necessary to obtain an increment of the moment situated at the domain boundary to be depended on only the moments within this domain. In this case, substitution of zero values for the moments situated out of the domain does not affect the increments of the moments within it. The comparison of calculations at different domains of summation is carried out:

- a triangular domain $R E G 1: l \leq N_{0}-r$,

- a triangular domain $R E G 2: r \leq\left(N_{0}-l\right) / 2$

- $\quad$ and a rectangular domain REG3: $r<R_{0}, l<L_{0}$.

Here, $R_{0}$ is the number of the Sonine polynomials, $L_{0}$ is the number of the Legendre polynomials in the DF expansion. For CEM-model, the domain REG2 is more real. The calculations show that, with the same amount of moments, the results are not worse than those for a domain $R E G 1$. The calculations for the triangular domains are more optimal than for the rectangular one. Nevertheless, to exclude the largest values of $l$ and thereby to diminish further amount of moments, summation can be carried out for a trapezoidal domain $R E G 4$

$l \leq N_{0}-r, \quad l \leq L_{0} \quad L_{0} \leq N_{0}$

with a relevant selection of $N_{0}$ and $L_{0}$. As a rule, further calculations are carried out with summation over a domain $R E G 4$. To build up the DF via moments, first of all $f_{l}(c)$ (DF in $l$ subspace) are to be calculated via the formula

$f_{l}(c)=M(c) \sum_{r} c^{l} S_{l+1 / 2}^{r}\left(c^{2}\right) C_{r, l}$,

then, via a formula

$$
f(c)=\sum f_{l}(c) P_{l}(\cos (\Theta))
$$

the DF is obtained.

Consequently, the numerical studies are carried out for the cases of the weak, moderate, and strong electric fields. These ranges are determined as follows:

- Weak electric field $-\epsilon \leq 0.1$,

- Moderate electric field $-0.1<\in<1$,

- $\quad$ Strong electric field $-\epsilon \geq 1$.

First, we dwell on the capabilities of the stationary moment method. In a domain of weak electric field, with the moment method, the stationary DF can be built-up in a rather wide range down to very small values of $10^{-22}$ order of magnitude. It is possible to perform in spite of the Grad criterion violation, but it is necessary to determine exactly a cut-off domain on $(r, l)$ plane when restoring the DF via its moments. An attempt to widen a convergence range beyond the optimum value of $R_{0}$ results in an opposite effect narrowing the convergence range. The optimum value of $R_{0}$ is within a neighbourhood of the minimum on a dependence $\left|C_{r, l}\right|(r)$. Transferring to the moderate field domain, the very fast decrease in minimum depth on a dependence $\left|C_{r, l}\right|(r)$ occurs with an increase in $\in$. An attempt to build up the stationary DF via the stationary moments gives no satisfactory results already at $\in=0.25$. In Fig. (2) a ratio of approximate $\operatorname{DF} f_{\Sigma}$ to exact solution is presented for $\epsilon=$ 0.25 . It shows the results of summation over domains $R E G 2$ at $N_{0}=1$ and $N_{0}=2$. The best result turns out to be when summing over $R E G 3$ domain at $R_{0}=2$. With an increase in $R_{0}$ and $L_{0}$, whole deal is only poorer. One can see that there is no success in approximation to the exact solution anywhere. The results obtained at larger values of $\in$ turn out to be much worse. In iteration method advanced by Kihara [25] to built up the stationary solution, the moments are sought. Emphasize that, for CEM-model, the stationary moments are known analytically. Nevertheless, the restoration of stationary solution is not possible with them. Hence, no hope exists to build up the stationary DF in a domain of moderate fields via iteration method.

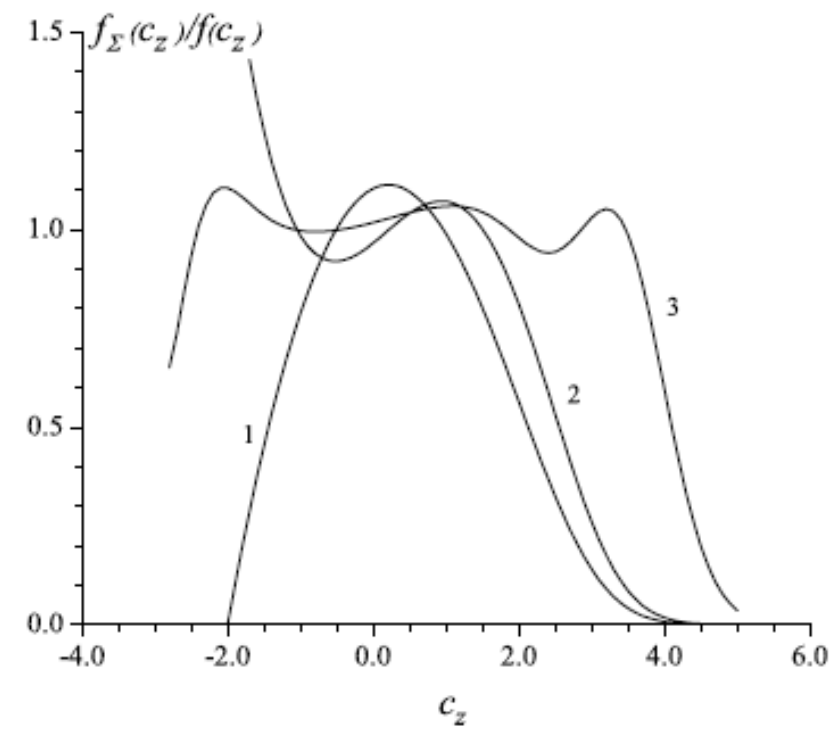

Fig. (2). The ratio of $f_{\Sigma}\left(c_{z}\right)$ to exact solution in the stationary state, $\varepsilon$ $=0.25$, CEM-model. Summation occurs in the domains REG2: Curve $1-N_{0}=1$; Curve $2-N_{0}=2$; Curve 3 refers to summation in a domain $R E G 3 R_{0}=2, L_{0}=6$.

The other deal is to restore the DF via its moments for the non-stationary process. It is demonstrated at switch-on of a field $\epsilon=0.5$ when the DF can not be restored via stationary moments.

At $t=3$, within a whole range $-10<c_{z}<10$, one can obtain a full coincidence with the exact solution for which purpose it is needed $R E G 3$ with $R_{0}=30, L_{0}=50$. By a moment $t=3$, within a whole domain of negative velocities, the DF reaches its stationary value limit and it experiences no changes at further increase in time. So, in the nonstationary method, there is no need in convergence in a domain of negative velocities. Hence, the summation region decreases essentially, i.e., $R_{0}$ and $L_{0}$ values. The dependences $f\left(c_{z}, t\right)$ on $t$ at constant $c_{z}$ are built up via exact formulas in Fig. (3) to demonstrate in what manner the establishment of stationary value $f\left(c_{z}\right)$ occurs. It is seen in Fig. (3) that in a domain of negative $c_{z}$ including $c_{z}=0$ a monotonous decrease in DF occurs in a course of relaxation process. The time needed for obtaining the stationary value does not exceed 3 and it is well below this value at the large negative values of $c_{z}$. For positive $c_{z}$, the relaxation process is non-monotonous with inherent delay of its start, and, at large $c_{z}$, longer time is necessary for obtaining the stationary value, e.g., at $c_{z}=5$, the process is over at $t \sim 10$. Hence, the stationary solution 
yield occurs nonuniformly over $c_{z}$. This relaxation feature is due to perturbation wave spreading in velocity space along a characteristic $c_{z}=c_{z}^{0}+\epsilon \cdot t$. This characteristic is obtained for the left differential side of the kinetic equation and then does not depend on a model of ion-atom interaction. So, one can expect that the relaxation process described above with front spreading in velocity space takes place not only in the case of CEM-model but at arbitrary ion-atom interaction potential.

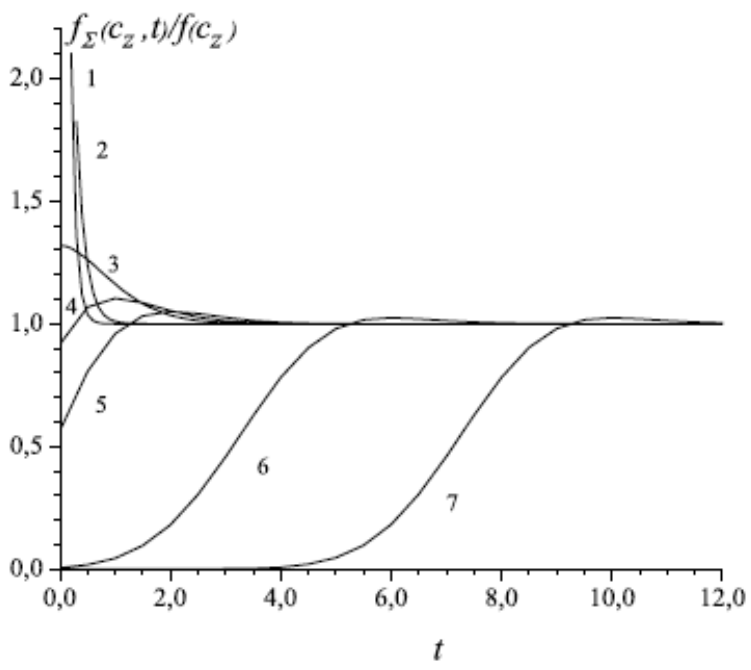

Fig. (3). Dependences of a ratio $f\left(c_{z}, t\right) / f\left(c_{z}\right)$ on $t$ at different $c_{z}, \in=$ 0.5: Curve $1-c_{z}=-10,2-c_{z}=-5,3-c_{z}=0,4-c_{z}=0.5,5-c_{z}=$ $1,6-c_{z}=3,7-c_{z}=5$.

An increase in the electric field $\varepsilon$ as well as in time $t$ results in poorer convergence of DF restoration process via its moments. It seems that the cause is in $\left|C_{r, l}\right|(r)$ representation. At any finite time, starting with certain $r$, the moments decrease. The maximum is observed on the dependences $\left|C_{r, l}\right|(r)$. With an increase in $t$, a coordinate of maximum and its height increase both with no limits. For a series (47) convergence to the exact solution, when summing

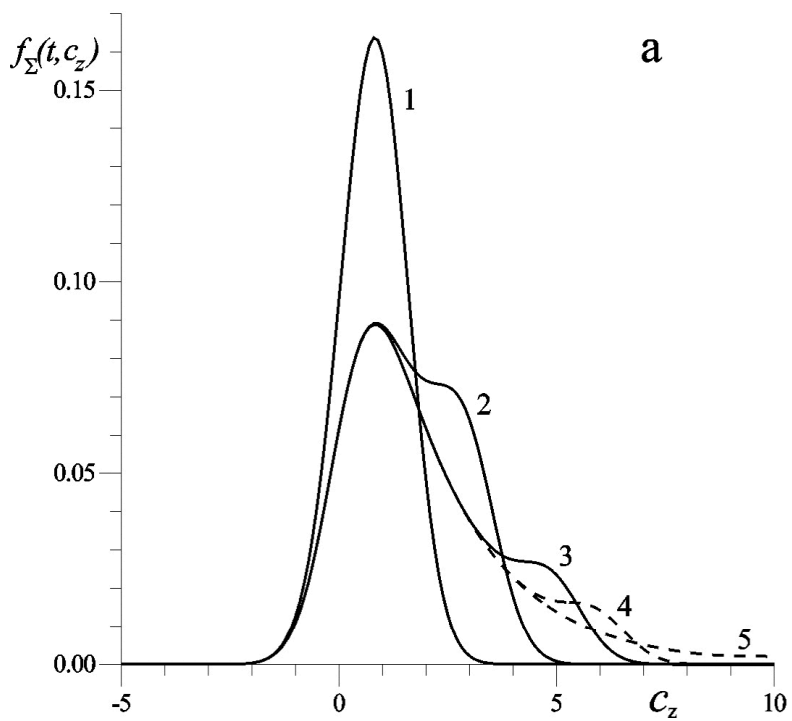

over $r$, it is necessary to go across the maximum and enter the $r$ domain where the values of $\left|C_{r, l}\right|$ are sufficiently small. At the longest times, when the coordinates of maximum are large, such a summation becomes questionable. However, in a domain of moderate electric fields, the DF can be successfully restored up to sufficiently long time. In a domain of strong electric field, to overcome the maximum becomes more complicated problem. The cut-off domain should be strongly enlarged in $(r, l)$-plane for convergence.

It is maintained that the dependences $\left|C_{r, l}\right|(r)$ turn out to be very similar to each other if $\epsilon$ and $t$ are changed simultaneously but the product $\in \cdot t$ being constant all the way. Thus, a similarity parameter $\in \cdot t$ is found. It is shown that a good convergence over all velocity range $-10 \leq c_{z} \leq 10$ under consideration takes place at $\in \cdot t<5$.

The DF for CEM-model is presented on the axis of symmetry $\left(c_{\rho}=0\right)$ at different times at $\epsilon=2$ in Fig. (4). It is shown in what manner the number of the Sonine polynomials $R_{0}$ and the Legendre polynomials $L_{0}$ increases with a time increase to be taken into consideration for the DF expansion. For $c_{z}>-4$, there is a fine coincidence with relevant ratio of the analytical solutions. For $c_{z}<-4$, the very fast outcome of the stationary solution occurs but with an increase in $t$ the solution via moment method becomes more difficult to build up. When a value in maximum $\left|C_{r, l}\right|(r)$ is higher than $10^{16}$, it becomes non-surmountable obstacle for summation in (47) using the standard computations. The procedures with larger decimal digits make possible to go farther (dashed curves in Fig. (4)).

Thus, using the non-stationary moment method, the solution in a domain of moderate and sufficiently strong electric fields is successfully obtained. The value of $\in$ (for which the DF is successfully built up for the stationary state) has been increased at least by one order of magnitude.

\subsection{Numerical Calculations for Different Models}

Consider all interaction models described in Section 2 consequently at the weak, moderate, and strong fields.

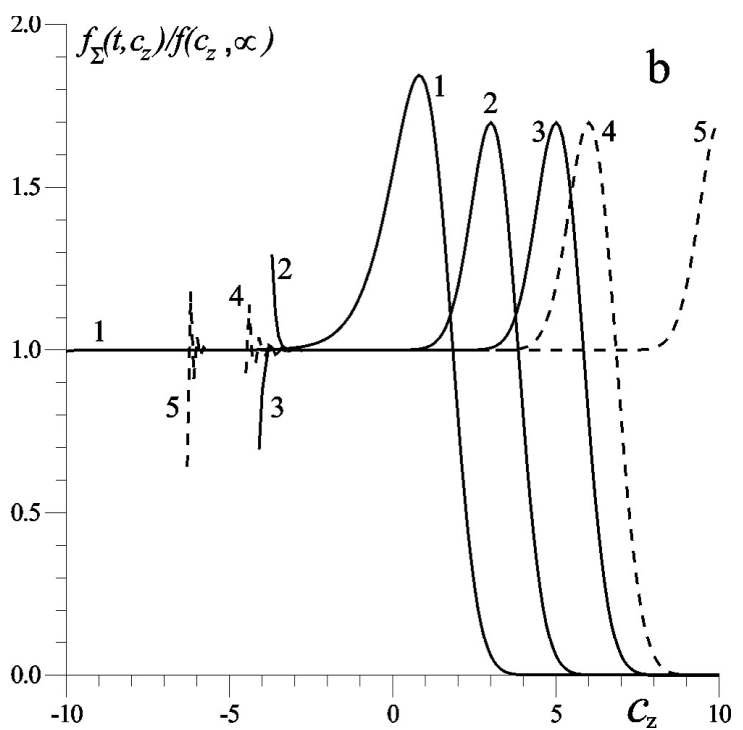

Fig. (4). Distribution function on axis of symmetry at different times for CEM-model at $\in=2.0$. (a) $-f\left(c_{z}, t\right)$. (b) $-f\left(c_{z}, t\right) / f\left(c_{z}, \infty\right)$. Curves $1-5$ refer to times $t=0.5\left(L_{0}=35, R_{0}=23\right) ; 1.5\left(L_{0}=30, R_{0}=26\right) ; 2.5\left(L_{0}=37, R_{0}=70\right) ; 3\left(L_{0}=75, R_{0}=100\right) ; 5\left(L_{0}=100, R_{0}=280\right)$. 


\subsubsection{Weak Electric Field}

Over all range of weak fields $(\epsilon \leq 0.1)$ for all models under consideration, mobility practically does not depend on $\epsilon$ and coincides with its value obtained via ChapmanEnskog method. This is not for case of the distribution function which turns out to be more sensitive to a value $\in$.

In the Chapman-Enskog method the DF is found at very small $\in$ as follows

$f(\mathbf{c})=M(c)(1+\in \varphi(\mathbf{c}))$.

Along with (49), $\varphi(\mathbf{c})$ can be determined with a $f(\mathrm{c})$ obtained numerically as follows

$\varphi(\mathbf{c})=\frac{1}{\epsilon}\left(\frac{f(\mathbf{c})}{M(c)}-1\right)$.

There is a domain of very small values of $\epsilon$ where $\varphi$, has been built up in such a way, that it does not depend on $\in$. We will call it the universal Chapman-Enskog correction.
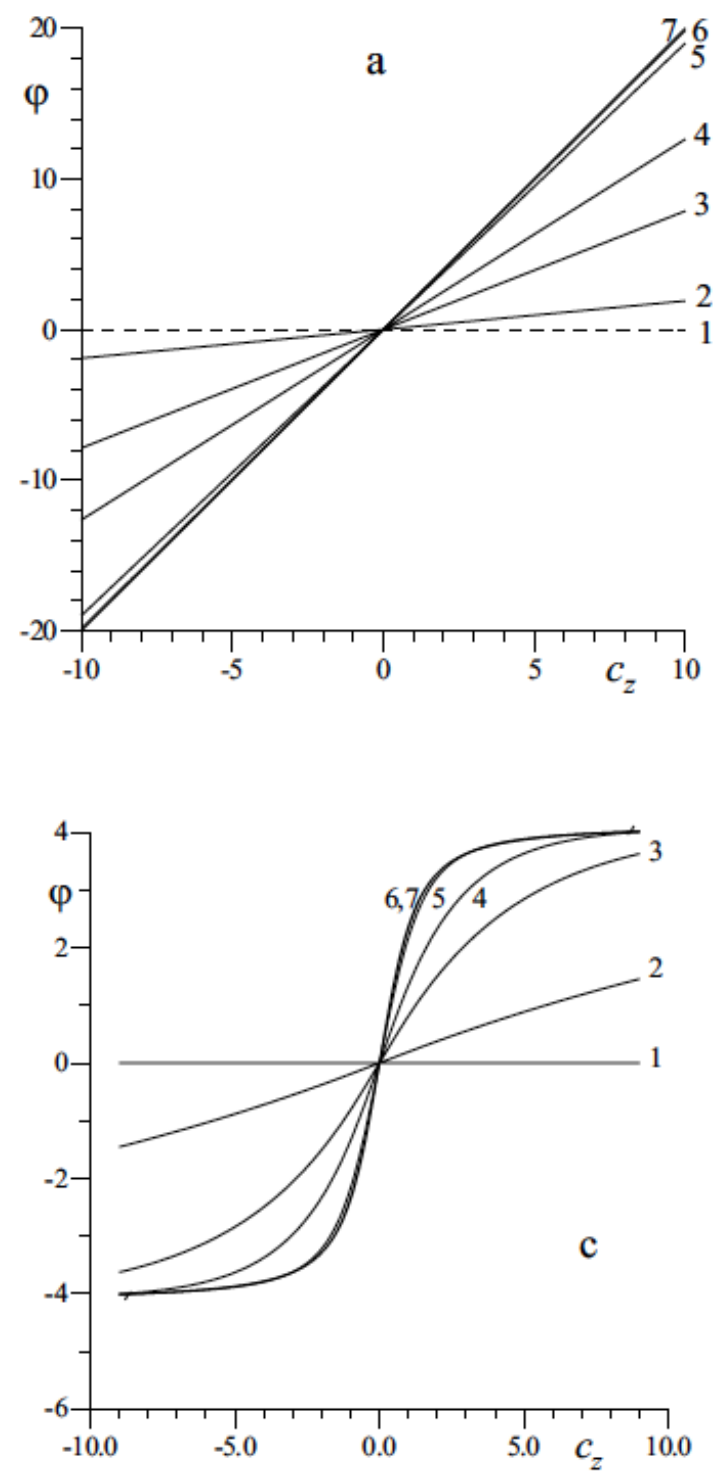

Fig. (5). Evolution of universal $\varphi$ at $c_{\rho}=0$. Non-standard normalization. (a) - CEM-model, (b) - pseudo-Maxwellian molecules, (c) CEHS-model, (d) - HS-model, Curves: $1-t=0,2-t=0.5,3-t=1,4-t=3,5-t=5,6-t=6,7-t=9.5,8-t=12,9-t=24$. Curves $5-9$ coincide with each other in this Figure.
We are certain that, the value $\in=10^{-4}$ for all interaction models is well within the domain where universal ChapmanEnskog correction is built up. In Fig. (5) evolution of universal $\varphi$ for four models is presented. For all models, a scale (unit for time measurement) has been chosen in such a way that mobility tends to unity at $\in \rightarrow 0$ and $t \rightarrow \infty$. We called this non-standard normalization.

Note, for CEM-model at $t \rightarrow \infty$, mobility $K \equiv 1$ at all values of $\epsilon$. For all other models we are doing the following: first, the non-stationary problem is solved with taken standard normalization (see subsection 1.3.3) and at sufficiently small $\epsilon$. In a wide range of small $\in$, a limit (stationary) value $K$ is constant.

Knowing this value $K$, it is easy to find the new frequency unit for which the stationary $K$ turns out to be unity. When transferring to this new frequency unit, all the standard MEs should be multiplied by the same constant denoted by $A$. Respectively, the time unit $\tau$ changes as well as a value of dimensionless electric field. In Table 1, the
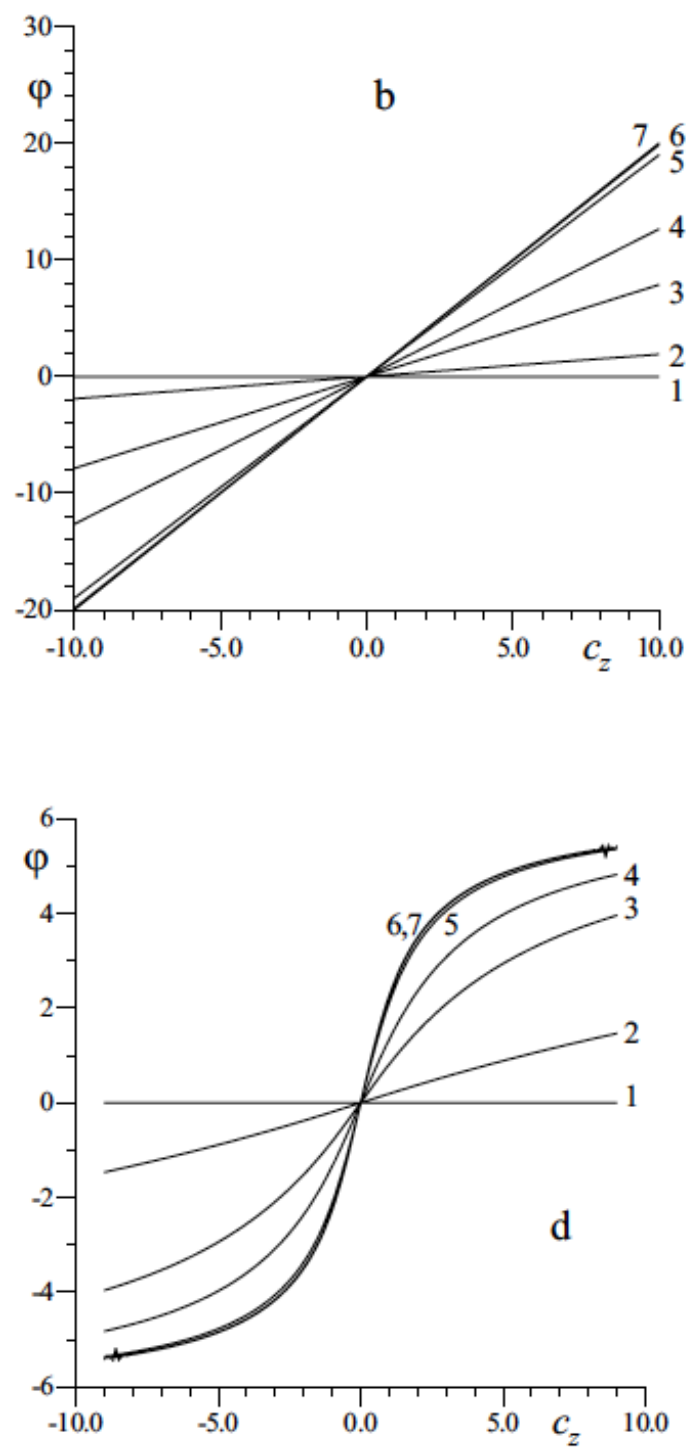
values of a coefficient $A$ are presented for different interaction models. From Table, one can see that a coefficient $A$ is strongly affected by the angular dependence of scattering cross-section. Thus, for the models with $\tau=$ const, a transfer from $180^{\circ}$-scattering to the isotropic one gives two-time rise in coefficient $A$. There is approximately twofold (exactly, 1.985) increase in $A$ when transferring from CEHS-model to HS-model.

Table 1. Transfer Coefficient from Standard to NonStandard Normalization $\boldsymbol{A}$ for Different Interaction Models. PMM Stands for Pseudo-Maxwellian Molecules

\begin{tabular}{|c|c|c|c|c|}
\hline CEM-model & PMM & CEHS-model & HS-model & Coulomb \\
\hline \hline 1 & 2 & 0.4826 & 0.9578 & 0.28128 \\
\hline
\end{tabular}

In Fig. (5a,b) one can see a coincidence of the results of CEM-model and pseudo-Maxwellian molecules in this normalization. This is due to the same dependence of the cross-section on velocity. In Fig. (5c,d), a comparison of two models with equal dependences of scattering cross-section on velocity (namely CEHS-and HS) is presented. Inherent for these models is a non-diagonal form of interaction matrix. Here, in spite of similar behaviour of $\varphi$, there is no identity of solutions.

For both models at long times, a tend to saturation is relevant at larger $c_{z}$. However, a value of such saturation is approximately 1.5 times smaller for CEHS-model compared with HS-model.

Nevertheless, of more important is the similarity of these solutions: first, very similar behaviour at small times; second, universal $\varphi\left(c_{z}\right)$ does not tend to $\infty$ with an increase in $c_{z}$ but to saturation, in contrast to the Maxwellian models. So, the Grad criterion is held for the DF at the stationary state for these models.

From the analytical solution of moment system for CEMmodel, we can conclude that $\varphi=2 c P_{1}(\cos \theta)=2 c_{z}$, coinciding with Fig. (5a). Universal Chapman-Enskog correction $\varphi$ is determined only by those moments $C_{r, l}$ which are proportional to $\in$. This subset is called $C_{r, l}^{(1)}$.

For numerical solution of moment system, a subset $C_{r, l}^{(1)}$ is sought as follows. The problem is solved at two values of $\in$ being within a domain of very small $\in$ (we select $\epsilon_{1}=10^{-3}$ and $\epsilon_{2}=10^{-4}$ ). Then all $C_{r, l}$ are divided by the corresponding $\in$. Those, for which $C_{r, l} / \in$, do not depend on $\in$ and refer to the original subset $C_{r, l}^{(1)}$. In addition, the groups $C_{r, l}$ of orders $\in^{2}$, $\epsilon^{3}$ and so on are obtained. If only moment $C_{01}$ contributes to the universal $\varphi$, for the case of the models with diagonal matrix, all the moments $C_{r, 1}$ contribute to $\varphi$ in the cases of other models. All $C_{r, l}$ at $l \neq 1$ have not any influence on $\varphi$.

In the case of weak field, in [9], a Kihara formula is involved for a ratio of mobilities in second and first approximation in terms of $r$. For HS-model it has a form
$\frac{[K]_{2}}{[K]_{1}}=1+\frac{1}{56}=1.01785$.

From our calculations for HS-model at $\left.t \rightarrow \infty,[K]_{2} / K\right]_{1}$ $=1.01727$ is obtained, being in a fine accordance with the result (51). If the cut-off of system of moment equation at $R_{0}$ $=1,2$ in the case of weak field is valid to calculate mobility, it is needed $R_{0}$ to be rather large for DF building-up. The study on effect of $R_{0}$ (the limit of summation over $r$ ) on $\varphi$ over the subspace $l=1$ is presented in Fig. (6).

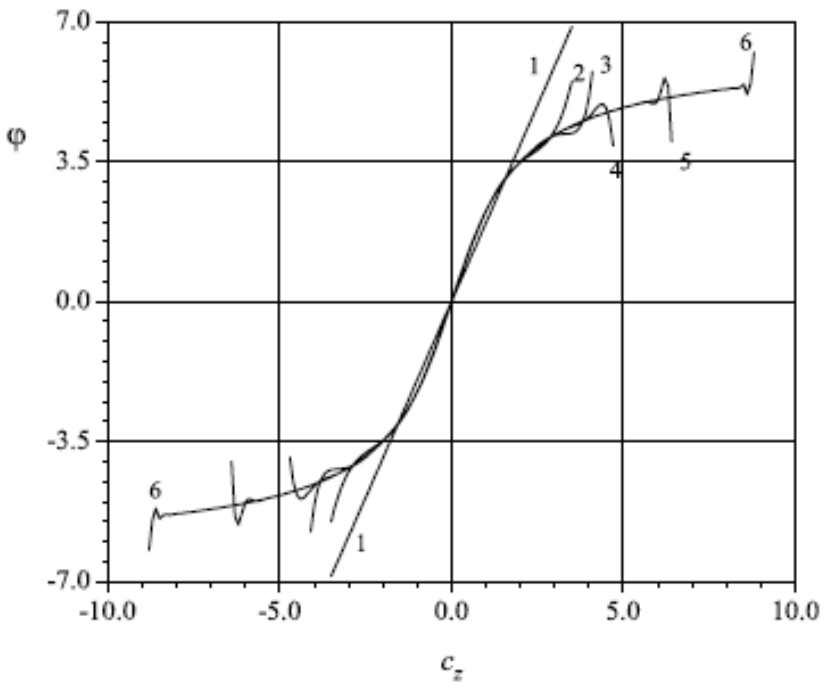

Fig. (6). Restoring $\varphi\left(c_{z}\right)$ for HS-model at different $R_{0}$. Nonstandard normalization. Curves: $1-R_{0}=0,2-R_{0}=2,3-R_{0}=4,4$ $-R_{0}=8,5-R_{0}=32,6-R_{0}=128$.

In Fig. (6) one can see that, even at $R_{0}=128$, there is a progress in building-up the Chapman-Enskog correction $\varphi$ up to 8.5 thermal velocities. The calculations show that, with an increase in $R_{0}$ from 128 to 250, a $\left|c_{z}\right|$ domain widening to $\left|c_{z}\right|=9.8$ occurs in which a good convergence is observed. Consider a matrix $\Lambda_{r, r_{1}, 1}$ for HS-model. A deflection from the diagonal is featured by a parameter $\Delta=r-r_{1}$.

In Fig. (7a) the dependences $\Lambda_{r, r_{1}, 1}$ on $r_{1}$ are built up at several values of $\Delta$. One can see that, with an increase in $r_{1}$, the diagonal MEs $\Lambda_{r_{1}, r_{1}, 1}$ increase monotonously in absolute value, reaching value $\sim-36$ at $r_{1}=500$.

The MEs $(\Delta=1,2)$ nearest to diagonal, being even slightly smaller, than the diagonal ones, increase monotonously also with an increase in $r_{1}$. The MEs become rather small at $\Delta=3$ and 4 . The MEs decrease with larger distances from the diagonal. The matrix elements $\Lambda_{r, r_{1}, 1}$ are the differences of MEs of the gain and loss terms of the collision integral. In Fig. (7b) the MEs of a gain term are built up with the same values of $\Delta$ as those for the full MEs in Fig. (7a). One can see that $\Lambda_{r, r_{1}, 1}^{+}$are much smaller than $\Lambda_{r, r_{1}, 1}$ and the gain MEs decrease as $\Lambda_{r, r_{1}, 1}$ increase with an increase in $r_{1}$. Hence, the main contribution in MEs is due to the loss MEs at large $r_{1}$.

A somewhat doubtful situation arises: the difficulties in building-up the solutions via moment method occur because of an increase in MEs of a loss term. At the same time, a loss 


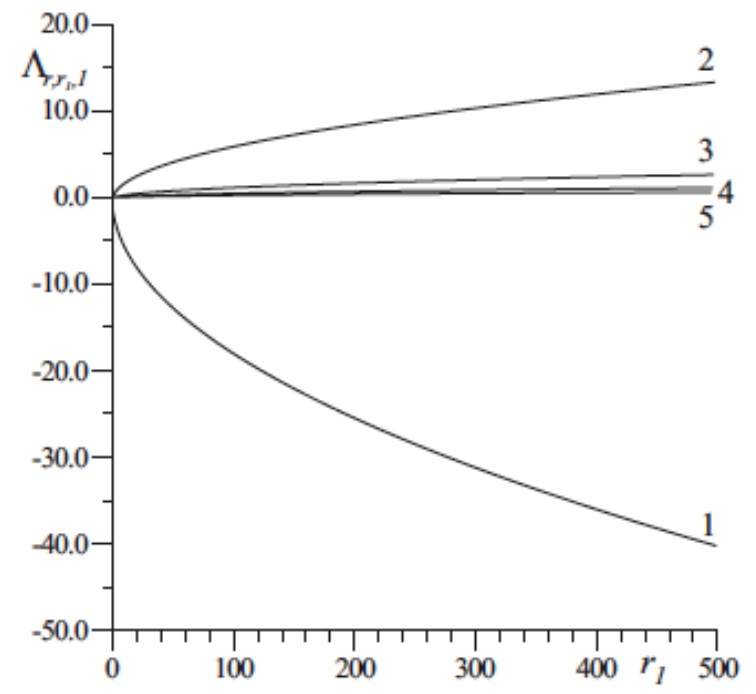

(a) Full MEs

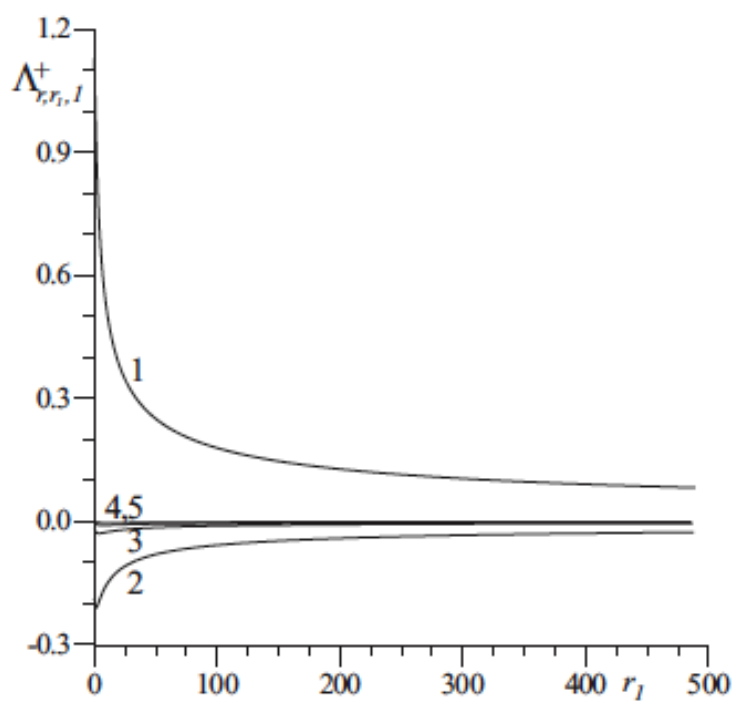

(b) Gain MEs

Fig. (7). Dependences of MEs of collision integral on $r_{1}$ for hard-sphere model. Curves: $1-\Delta=0,2-\Delta=1,3-\Delta=2,4-\Delta=3,5-\Delta=4$.

term and its MEs are the simplest part of the collision integral. In Chapman-Enskog approximation, a correction $\varphi$ is proportional to $P_{1}(\cos \theta)$. As a result, $\varphi\left(c_{z}\right)$ turns out to be an odd function on the axis of symmetry. Naturally, the question arises: up to what values of $\varepsilon$ a function $\varphi$ conserves this property? Already at $\in=10^{-2}$, the symmetry relative to a point 0 vanishes. At $t=10$ we have $\varphi(8)=5.59$ and $\varphi(-8)=-5.08$. Now, this is due to non-insignificance of $\varphi_{0}$ and $\varphi_{2}$. A more significant deflection from universal $\varphi$ is observed at $\in=5 \cdot 10^{-2}$ :now, at $t=10$, we have $\varphi(8)=6.75$ and $\varphi(-8)=-4.32$. It means that contribution from $\varphi_{0}$ and $\varphi_{2}$ becomes even larger, and other $\varphi_{l}$ can influence too at $l>$ 2.

\subsubsection{Moderate and Strong Electric Fields}

Conditionally speaking, the boundary of the transfer from weak to moderate electric field is $\epsilon=0.1$, and a lower boundary of strong field domain is $\epsilon=1$. The calculation results for different models are given. First, we follow mobility evolution, then consider the distribution functions. For the models with $\tau=$ const, the proportionality of a drift velocity $u_{d}(t)$ to the electric field $\in$ is relevant, i.e., independence of mobility $K$ on $\in$. For pseudo-Maxwellian molecules in standard normalization, the stationary value of mobility turns out to be two and a process toward stationary state is strongly dragged out compared with CEM-model. In non-standard normalization not only the stationary value of $K$ equals unity but a dependence $K(t)$ coincides totally with analogous dependence for CEM-model.

Go to the models with $\lambda=$ const. For such models the interaction matrix is non-diagonal. Consider HS-and CEHSmodels.

One can see in Fig. (8), in non-standard normalization, that mobility tends to the standard value of unity at small $\in$. However, with an increase in $\in$ the stationary value of
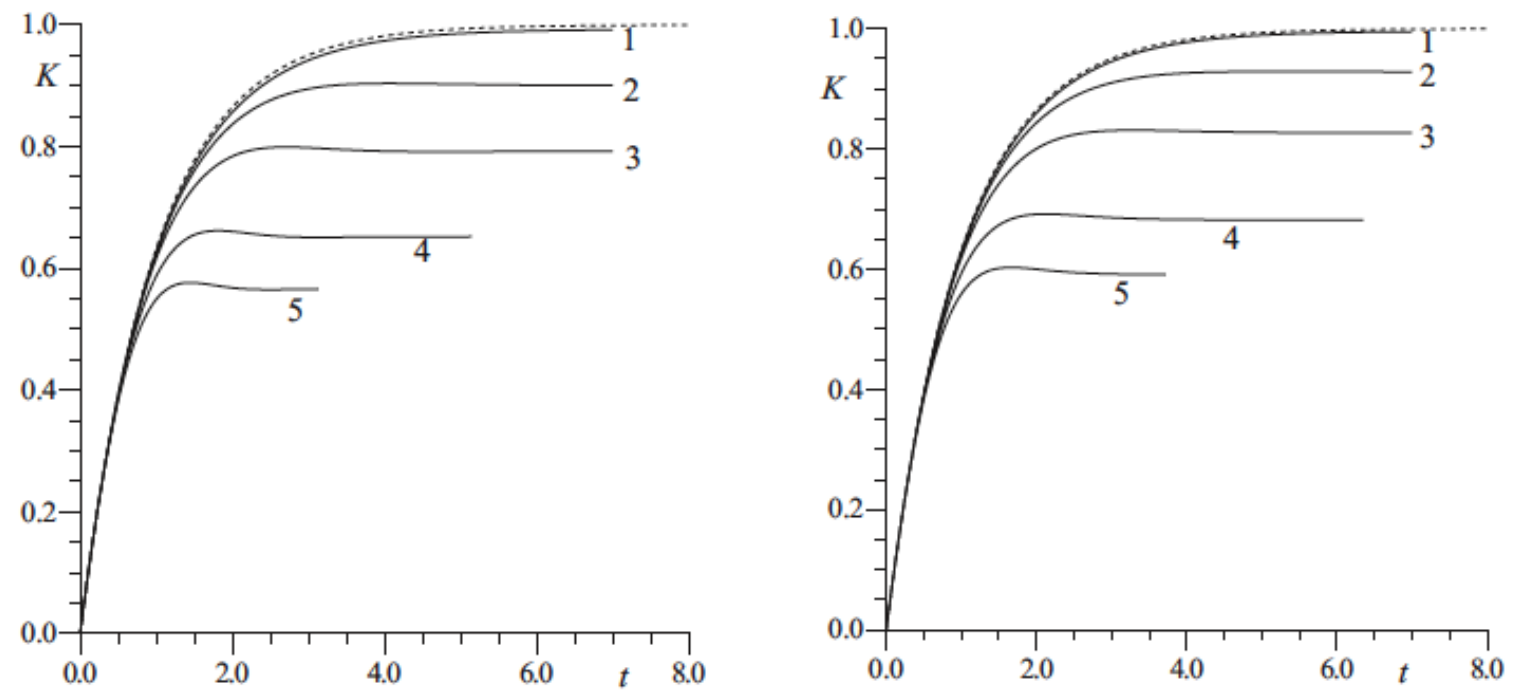

Fig. (8). Mobility $K(t)$ for several $\in$, (a) CEHS-model. (b) HS-model. Non-standard normalization. Dashed curve refers to CEM-model. Curves $1-\epsilon=0.1,2-\epsilon=0.5,3-\epsilon=1,4-\epsilon=2,5-\epsilon=3$. 
mobility decreases. A new interesting phenomenon is observed at sufficiently large value of $\in$. Non-monotonicity arises on the dependences $K(t)$ in contrast to the models with diagonal interaction matrix. The dependence $K(t)$ for $\in=1$ is presented for CEHS-model in Fig. (9) on enlarged scale. At the beginning, the mobility reaches its maximum with rise in time, then, goes across the minimum. A similar picture is observed in the case of HS-model. It can be assumed that the switch-on process is accompanied by exciting oscillation rapidly damped. The maximum moves toward smaller times and the value of maximum-minimum drop increases with an increase in $\in$. Till now we have considered a temporal dependence of mobility. Now, dwell on a behaviour of stationary mobility.

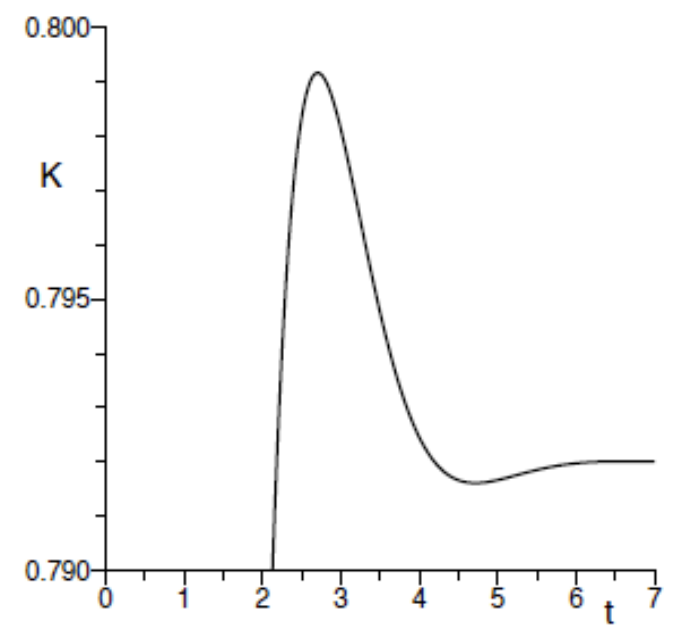

Fig. (9). $K(t)$ for $\epsilon=1$ on an enlarged scale, CEHS-model. Nonstandard normalization.

The mobility dependences on $\in$ at the stationary state in non-standard normalization are presented in Fig. (10). It can be noted a well pronounced dependence of $K$ on the electric field strength for both models. Mobility decreases monotonously always being below the standard ones. The analytical solution of the stationary Boltzmann equation is obtained for CEHS-model in the case of cold background gas in Perel's study [7]. In this case, a background gas

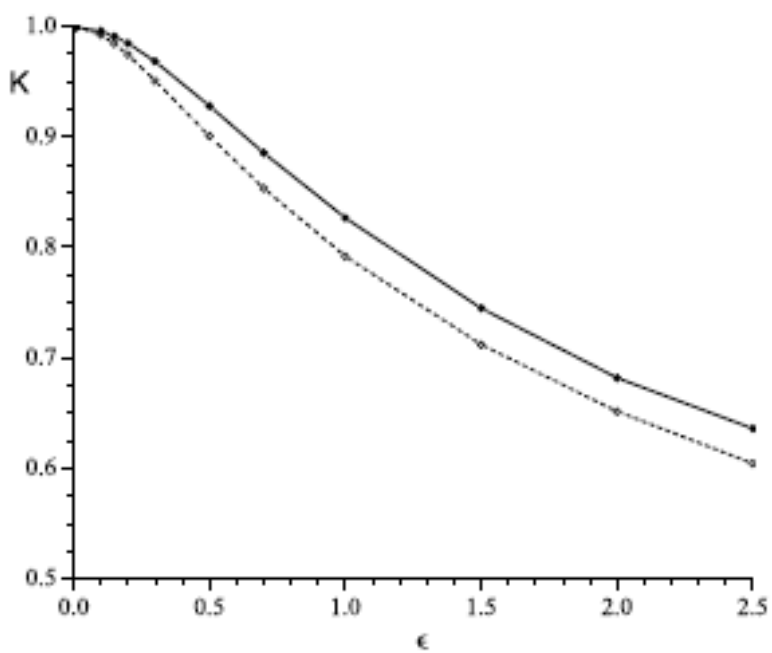

Fig. (10). Stationary mobility $K(\in)$. Solid curve refers to HSmodel. Dashed curve - CEHS-model. temperature tends to zero corresponding to the very strong field, the Maxwellian of the background being substituted by $\delta$-function and a collision operator being strongly simplified. The DF and mobility of ions was built up in [7]. In our denominations, this solution takes a form

$K(\in)=\sqrt{\frac{2}{\pi \epsilon}}$.

In Fig. (11) it is shown in what manner the stationary mobility $K(\in)$, built up via moment method, tends to the mobility limit determined by a formula (52).

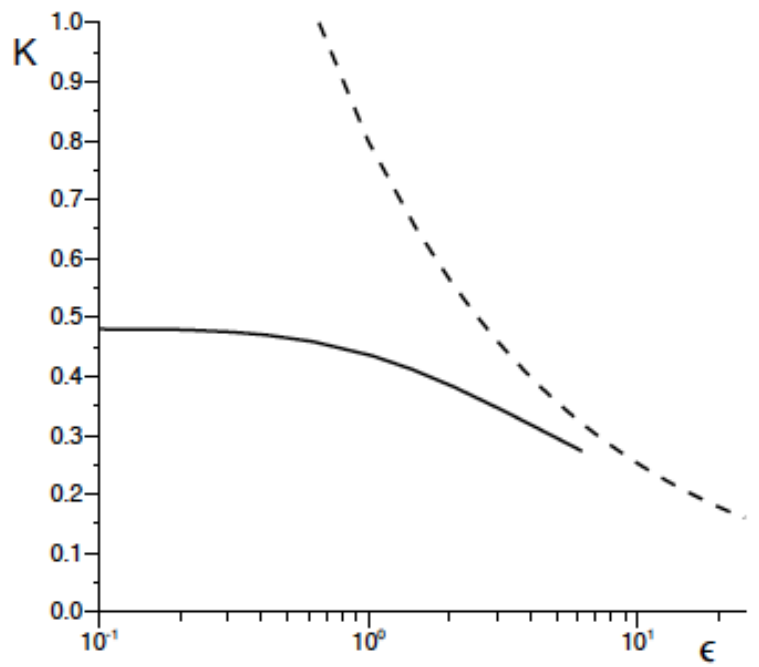

Fig. (11). Stationary mobility $K(\in)$ for CEHS-model. Comparison with Perel's solution. Standard normalization. Dashed curve refers to cold gas mobility.

Emphasize, that for four models under consideration mobility tends to saturation at large $t$. A totally different situation occurs in the case of Coulomb interaction. A mobility behaviour is shown at different $\epsilon$ in Fig. (12). For this model, mobility turns out to be higher than standard.

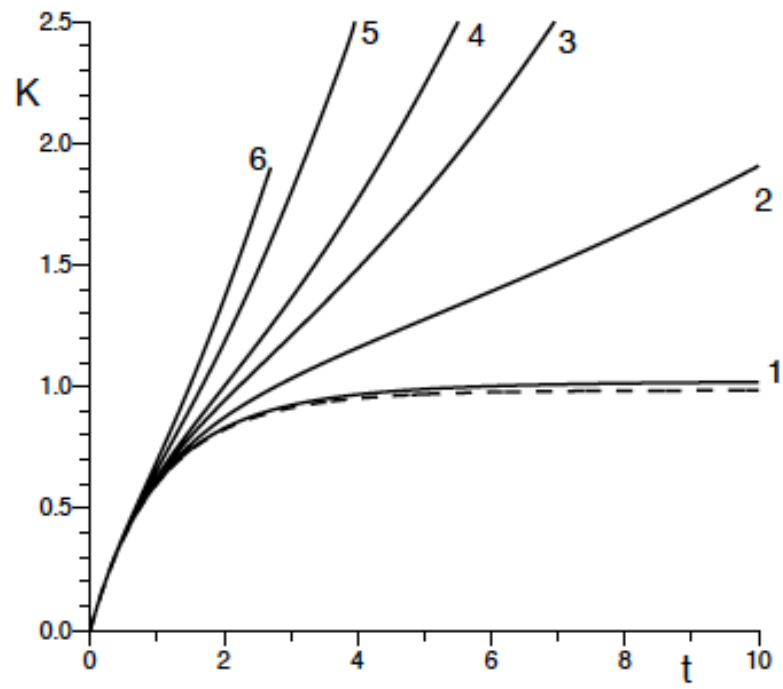

Fig. (12). Dependences $K(\in)$ for Coulomb interaction at different $\epsilon$. Dashed curve refers to the standard mobility. $1-\epsilon=0.1,2-\epsilon$ $=0.5,3-\epsilon=0.8,4-\epsilon=1,5-\epsilon=1.5,6-\epsilon=2$. 


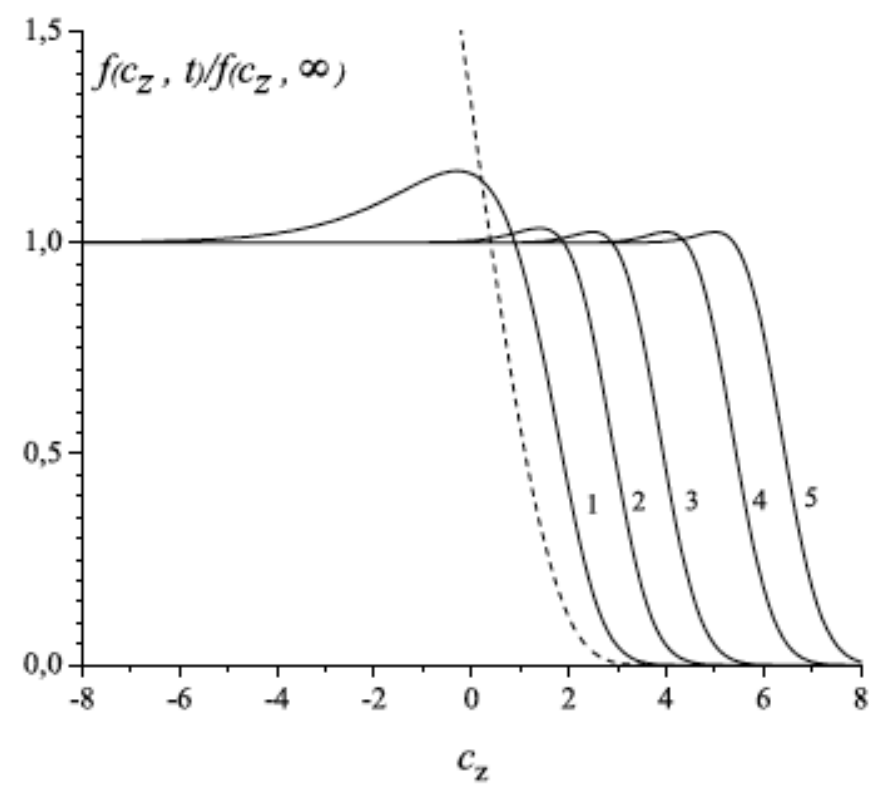

(a) CEM-model

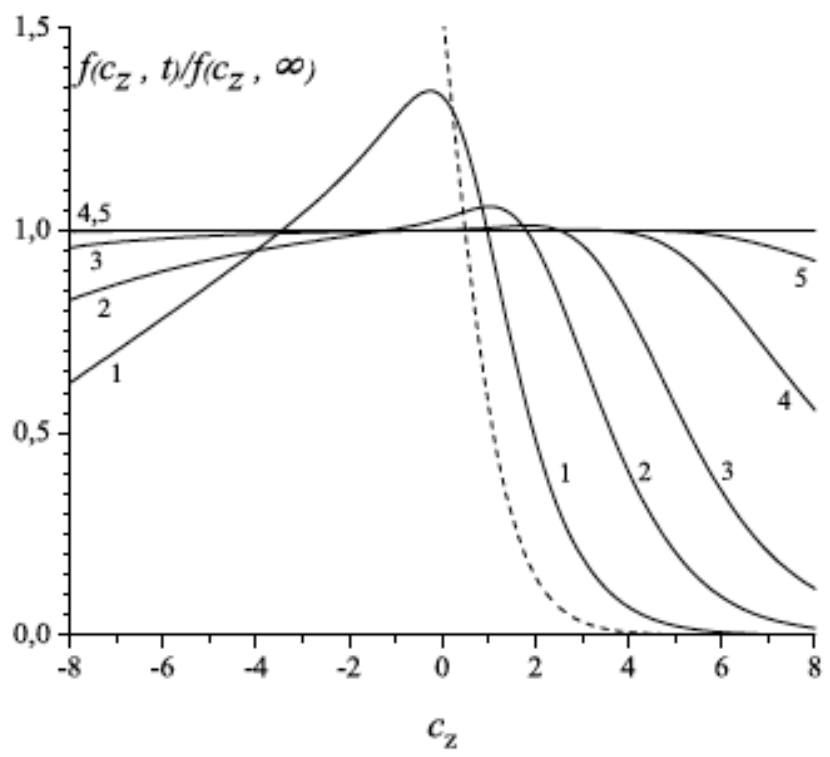

(b) HS-model

Fig. (13). Comparison of relative DFs at axis of symmetry at different times for CEM and HS-models at $\in=0.5$ in standard normalisation. $L_{0}=64, N_{0}=128$. Dashed curve refers to $t=0$. Curves: $1-t=1,2-t=3,3-t=5,4-t=8,5-t=10.5$.

Moreover, according to the calculations, it tends to an infinite growth already at $\in=0.3$; at smaller values of $\in$, this effect does almost not show itself; being more pronounced at $\in>0.3$.

As was mentioned above, this effect is because of ion runaway and it occurs due to strong decrease in scattering cross-section with a growth of relative velocity $\left(\sigma \sim g^{-4}\right)$.

Thus, mobility evolution is investigated for different interaction laws from the most rigid interaction potential (hard-sphere model) to the softest (Coulomb interaction).

In a domain of weak electric field, in non-standard normalization, mobilities are very similar to each other at all scattering cross-sections. In the case of Coulomb interaction, runaway of ions takes place. This process enchances with increase in $\in$.

Now to the distribution function. Here are given the calculation results on the DF for above mentioned models. The DF is built up not only on axis of symmetry at $\mathrm{c}_{\rho}=0$ but over an angle $\theta$ at several fixed values of a velocity $c$. All calculations are performed in non-standard normalization. In the case of CEM-model we follow evolution of a ratio of the DF to that at stationary state. Such representation of solution gives opportunity to clarify the interesting processes, particularly, in a domain of high velocities. For CEM-model, such ratio is built up very simple as the analytical solution
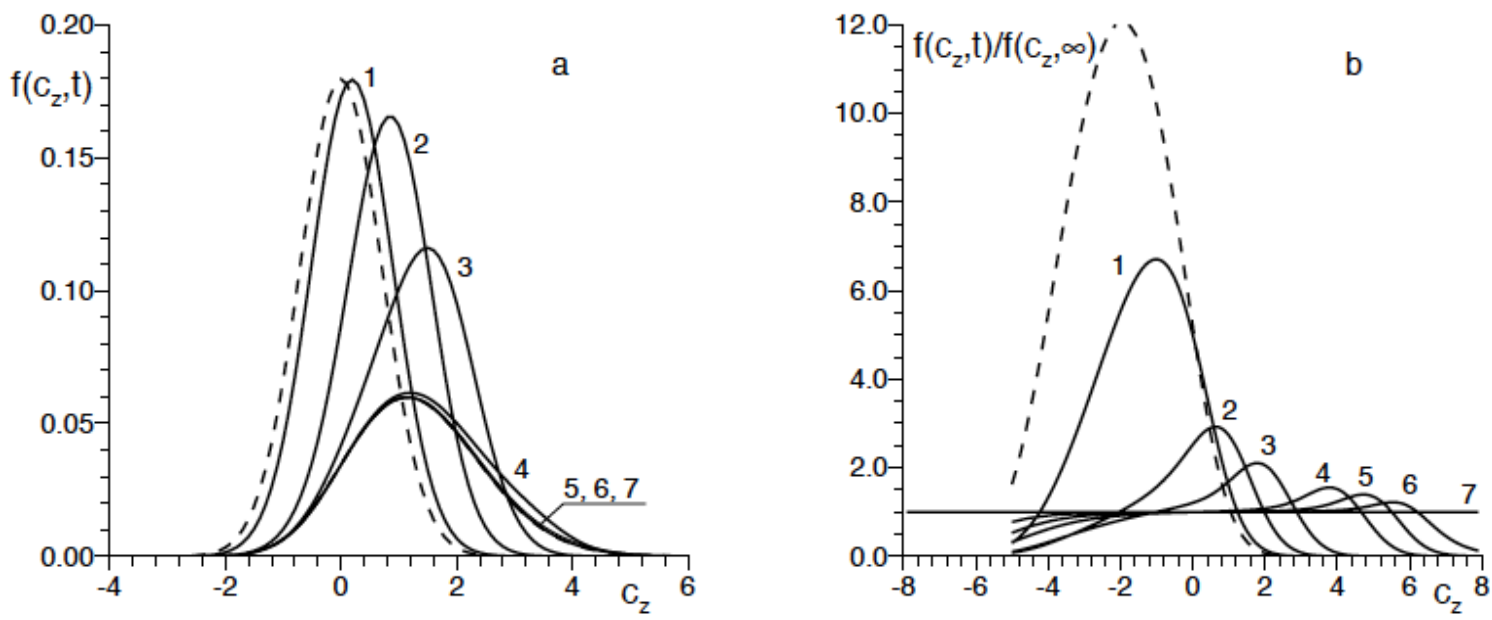

Fig. (14). Distribution function on axis of symmetry at different times for HS-model at $\in=2.0$ in non-standard normalization. $L_{0}=128, N_{0}=$ 128. (a) $-f\left(c_{z}, t\right),(\mathbf{b})-f\left(c_{z}, t\right) / f\left(c_{z}, \infty\right)$. Curves: $1-t=0.1,2-t=0.5,3-t=1,4-t=2,5-t=2.5,6-t=3,7-t=3.5$. Dashed curve refers to $t=0$. Curves $5-7$ coincide in (a). 


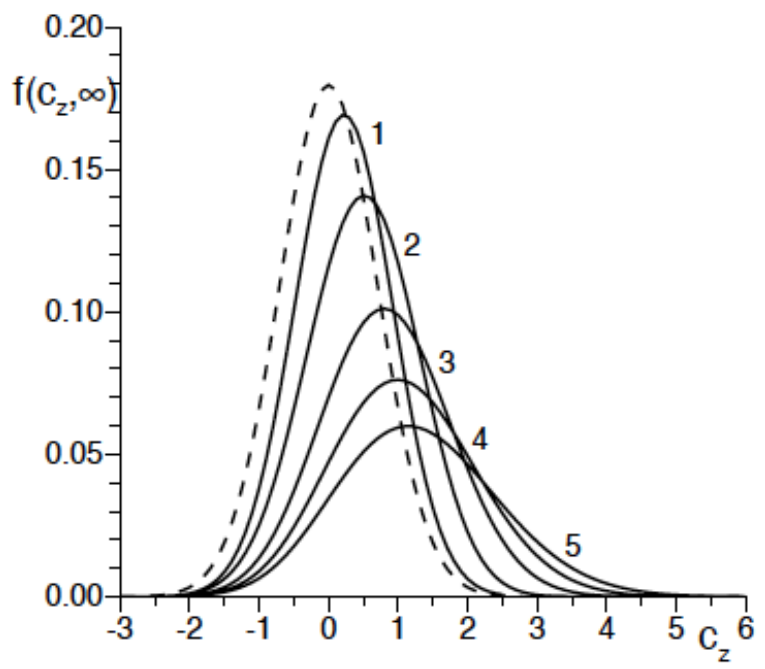

Fig. (15). Stationary distribution function $f\left(c_{z}, \infty\right)$ at different $\in$ for HS-model in non-standard normalization. $L_{0}=128, N_{0}=128$. Curves: $1-\epsilon=0.2,2-\epsilon=0.5,3-\epsilon=1,4-\epsilon=1.5,5-\epsilon=2$. Dashed curve refers to $t=0$.

exists for the stationary state. When building up the relative DF for other models, we use the numerical solution for sufficiently large time as the stationary one.

In the case of HS-model, the DF relaxation within velocity range under consideration $\left(c_{\rho}=0,-8 \leq c_{z} \leq 8\right)$ proceeds clearly more uniformly compared with CEM-
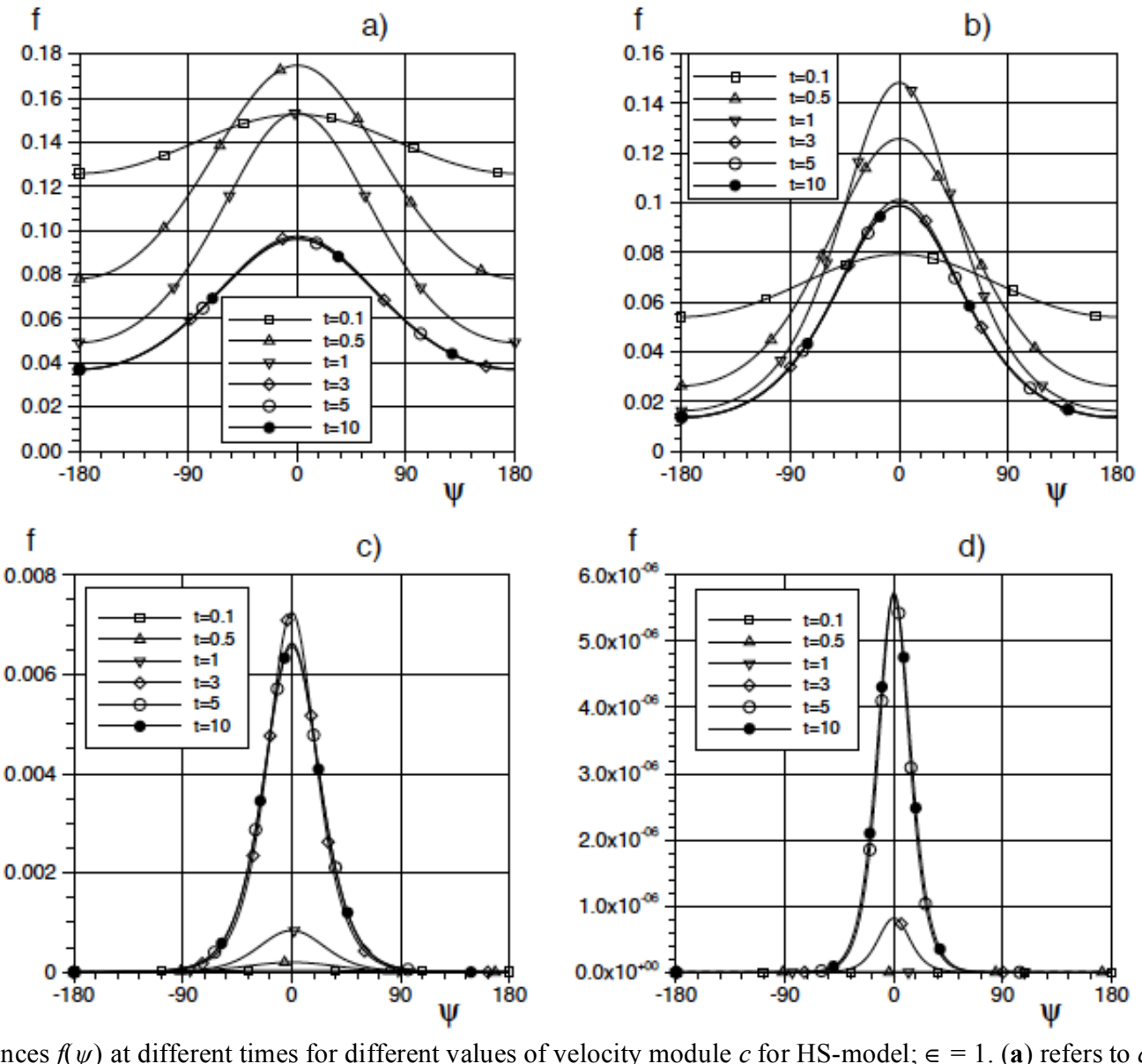
state terminates only by a time $t=15$. 14). Here, the stationary DF is that at $t=3.5$. draft the ways to overwhelm these difficulties.

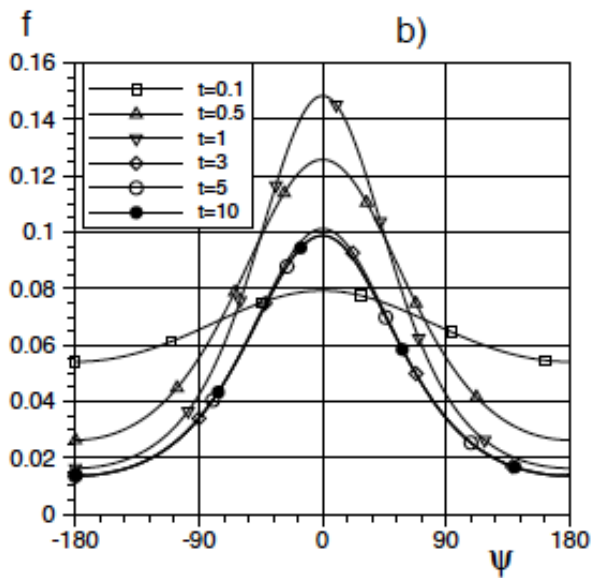

Fig. (16). Dependences $f(\psi)$ at different times for different values of velocity module $c$ for HS-model; $\in=1$. (a) refers to $c=0.5$; (b) $-c=1$; (c) $-c=3 ;(\mathbf{d})-c=5$.

model. Thus, at $\in=0.5$ (Fig. (13a)) in a domain of negative velocities, by $t=1$ the relaxation process has already terminated and the DF has reached its stationary state, as for HS-model (Fig. (13b)) in a domain $c_{z}<0$, the relaxation terminates only at $t=8$. By a time $t=11$, in the case of HSmodel, relaxation is over within all the velocity range. But, in the case of CEM-model, an approach to the stationary

At $\in=1.5$ the DF can not be built up successfully at $t=$ 10. There is no convergence over all velocity range under consideration. The solutions at $t=3$ and $t=5$ coincide practically at $\left|c_{z}\right|<6$. The DF at $t=5$ is selected to be $f\left(c_{z}, \infty\right)$. Even more pronounced are the listed effects at $\epsilon=2$ (Fig.

The stationary DF at different $\epsilon$ is shown in Fig. (15). One can see in what manner occurs more and more ion heating and how their progressively smaller number falls in a domain of negative velocities with an increase in the electric field strength. The ion temperature becomes higher and, at large $\in$, the DF is restored with more difficulties via its moments using the standard moment method. Below, we

If the distribution of velocities in transverse direction (over $c_{\rho}$ ) remains Maxwellian for CEM-model, the ion heating occurs in transverse direction for other models. So, it is of interest to build up the DF dependences on an angle in $\left(c_{x}, c_{z}\right)$-plane (i.e., $\left.c_{y}=0\right)$ together with the DF presentation on 


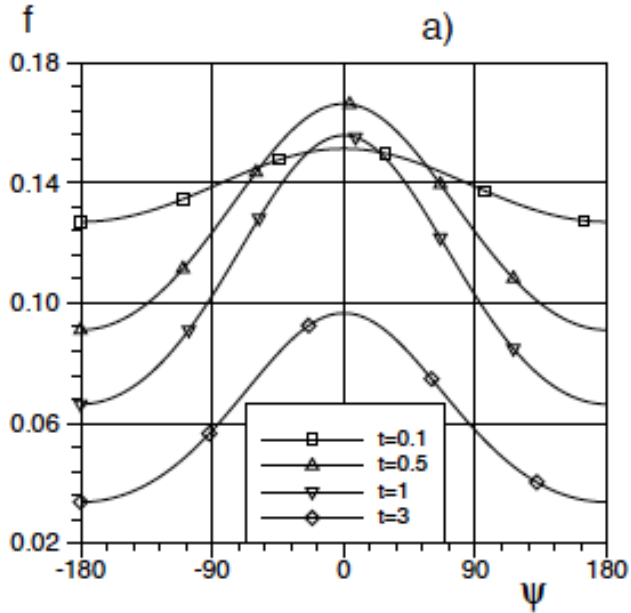

$\mathrm{f}$

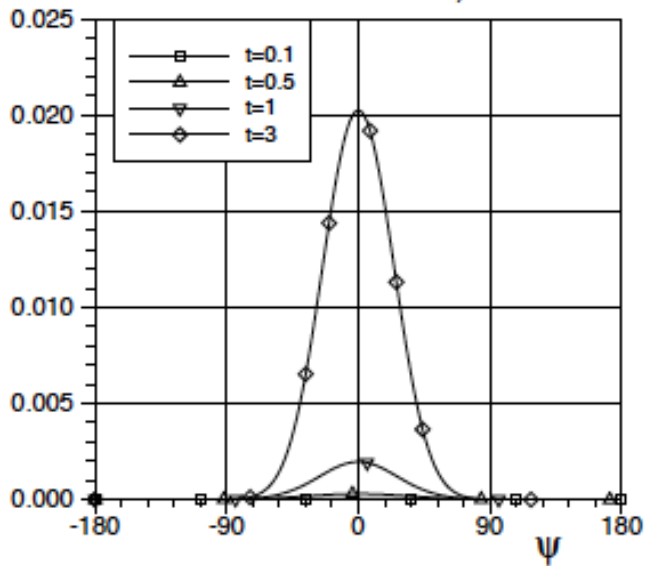

f

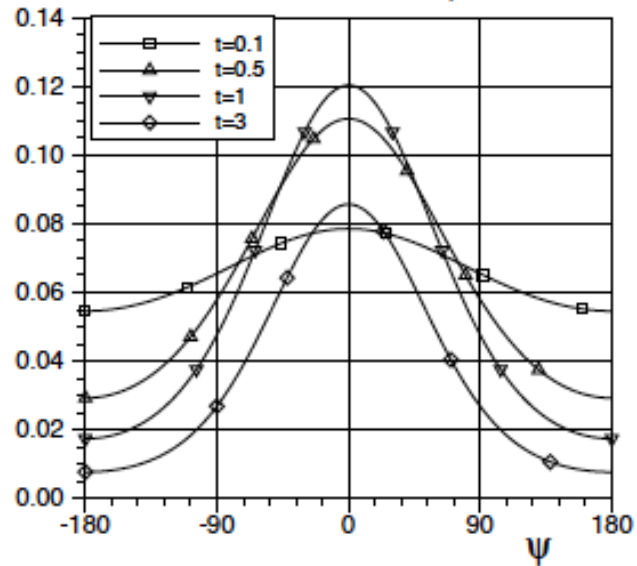

f

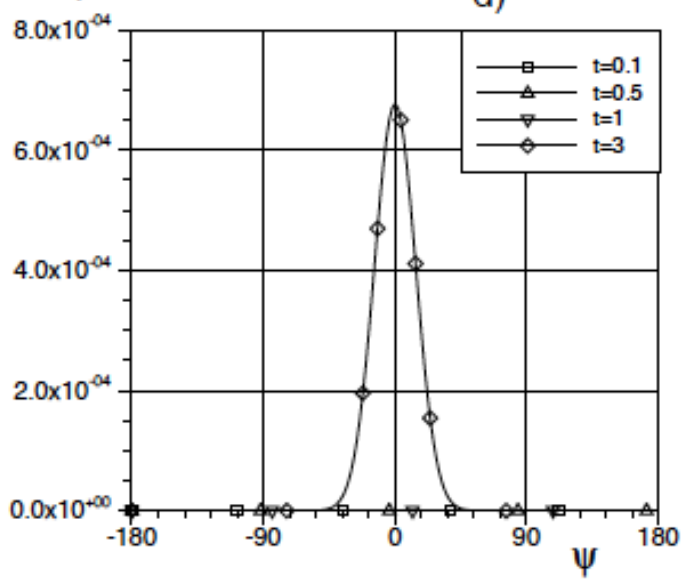

Fig. (17). Dependences $f(\psi)$ at different times for different values of velocity module $c$ for Coulomb interactions. (a) refers to $c=0.5$; (b) $-c$ $=1 ;(\mathbf{c})-c=3 ;(\mathbf{d})-c=5$.

axis of symmetry at several values of velocity module $c=$ $\sqrt{c_{x}^{2}+c_{z}^{2}}$. A rotation angle in $\left(c_{x}, c_{z}\right)$-plane is denoted by $\psi$. While $0 \leq \psi<\pi$, it refers to $\varphi=0$ and $\psi=\Theta$ in spherical frame of coordinates. For $-\pi \leq \psi<0$ we have a correspondence of $\varphi=\pi$ and $\psi=-\Theta$. Detailed calculations are carried out for three interaction models: HS-model, CEM-model, and Coulomb interaction. As an example, we show only two (Figs. (16) and (17)) corresponding to two opposite cases: the most rigid model (HS-model) and softest (Coulomb interaction) and for only value of the electric field, $\epsilon=1$. In these figures, one can see that a hight of maximum does well depend on an interaction model, especially, in a domain of high velocities. Thus, e.g., a transfer from HSmodel to the Coulomb interaction results in an increase in maximum by approximately two orders of magnitude at $c=$ 5 and $t=3$.

In the case of the Coulomb interaction, a point is under consideration in what manner the runaway effect influences the DF evolution. This effect reveals itself as a constant and rather fast growth of maximum on a dependence $f(\psi)$ in a domain of high velocities. This problem is rather troublesome and using the standard moment method and rather quickly we come up against a situation when there is no DF expansion convergence. A transfer to other basis is accomplished to improve the convergence. We use the Maxwellian with higher temperature instead of expansion about the weighted Maxwellian with atom temperature. Such approach we call the modified moment method. In more details it will be described in next Section.

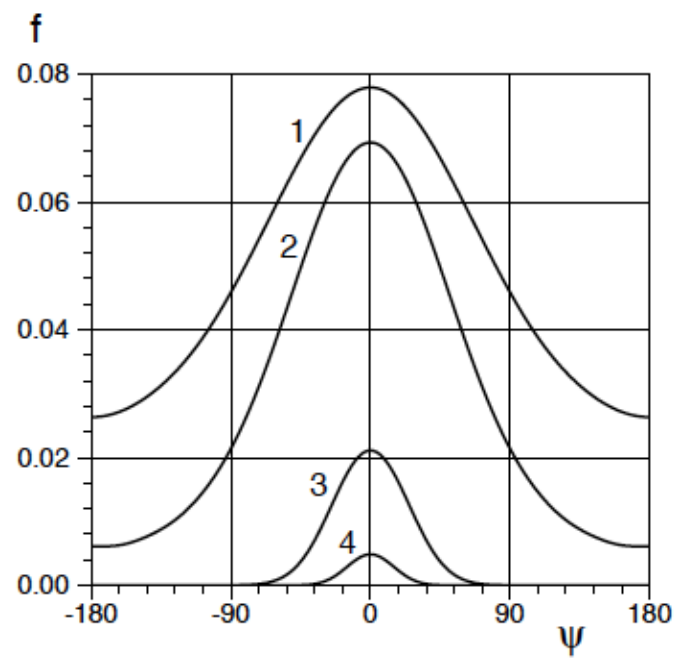

Fig. (18). Distribution function $f(\psi)$ at $\in=1$ and $t=4$ for the Coulomb interaction. Curve 1 refers to $c=0.5 ; 2-c=1 ; 3-c=3$; $4-c=5$. 
The calculations of ion runaway using the modified moment method are shown in Figs. (18) and (19). In Fig. (18), one can see that, the distribution function at $t=4$ in a domain of very high velocities at $\psi=0$ (i.e., in direction along the electric field) is sizeable with the DF in a domain of thermal velocities. In Fig. (19), it is shown in what manner the maximum value of the DF changes in time at $c=$ 3 and $c=5$. In spite of progress in time only by unity, when using the modified moment method, the important information is obtained concerning the runaway of ions. E.g., in a time from $t=3$ to $t=4$, at $c=5$, maximum value of the DF increases almost by one order of magnitude.

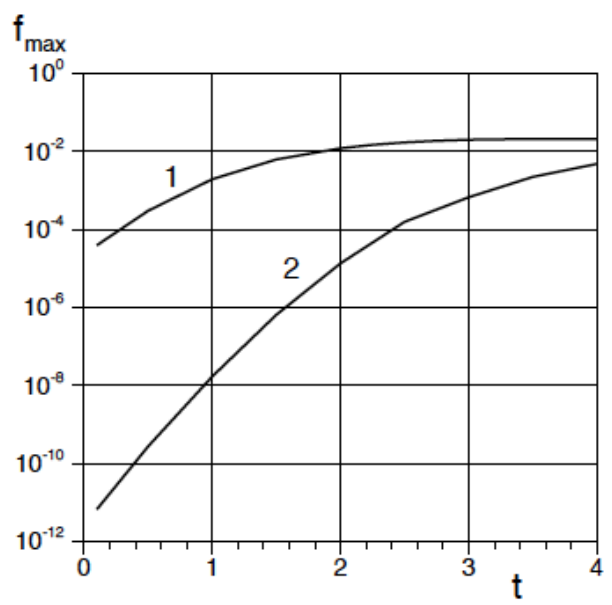

Fig. (19). Temporal dependence of maximum values of $f(\psi)$ for two values of $c$ at $\in=1$ for the Coulomb interaction. Curve $1-c=$ 3 , Curve $2-c=5$.

\subsection{Ways to Ameliorate Convergence}

Consider two ways to ameliorate the DF expansion convergence in terms of its moments. The first is DF expansion in terms of the spherical Hermite polynomials but with changed temperature of the weight Maxwellian. The second is DF expansion only in terms of the spherical harmonics.

\subsubsection{Modified Moment Method}

Consider a way to ameliorate the moment method convergence involving a change in the basis - a rise in temperature of weight Maxwellian. It was mentioned earlier in $[10,11,14]$ about the need in basis change. However, these works deal with calculation of complicated integrals when building up the MEs in a new basis. It resulted in enormous consumption of computer time [11] and sometimes to insufficient accuracy. At the same time MEs were built up with small indices only $(l \leq 7, r \leq 10)$. Consider the DF in two bases with temperatures of the weight Maxwellians $T_{0}$ and $T_{1}$. Vectors $\mathrm{C}^{0}$ and $\mathrm{C}^{1}$ are interrelated by (18) when transferring from a basis $T_{0}$ to $T_{1}$. A transfer matrix $D_{r, k, l}$ for this situation is as follows [3]

$$
D_{r, k, l}=\frac{(-1)^{r+k} k !}{r !} \frac{T_{0}^{r+l / 2}}{T_{1}^{k+l / 2}} \frac{\left(T_{1}-T_{0}\right)^{k-r}}{(k-r) !} \text {. }
$$

Denote a ratio of the temperatures of the bases as $R T=$ $T_{1} / T_{0}$. We call the modified moment method such one for which a parameter $R T$ is not unity. Generally speaking, this parameter can experience a change in time. The optimum dependence $R T(t)$ can be sought from a condition of minimum number of the moments well representing the DF.

If the Maxwellian in the collision integral is expanded in terms of a new basis, the formulas for the MEs $\bar{\Lambda}_{r, r_{1}, l}$ in this basis at non-coincidence of temperatures $T_{1}$ and $T_{0}$ can be obtained as follows

$$
\begin{aligned}
\Lambda_{r, r_{1}, l}\left(T_{1}\right)= & \frac{1}{g_{r, l}} \sum_{k}\left(1-\frac{T_{0}}{T_{1}}\right)^{k} \int H_{r, l}\left(\frac{m v^{2}}{2 k T_{1}}\right) \hat{I}\left(M H_{r_{1}, l}\left(\frac{m v^{2}}{2 k T_{1}}\right),\right. \\
& \left.M H_{k 0}\left(\frac{m v^{2}}{2 k T_{1}}\right)\right) d^{3} v \\
= & \frac{1}{g_{r, l}} \sum_{k}\left(1-\frac{T_{0}}{T_{1}}\right)^{k} K_{r_{1}, l, r-r_{1}, 0}^{r, l}\left(T_{1}\right)
\end{aligned}
$$

Thus, the linear MEs in the basis, which temperature distincts from atom temperature, are easily expressed via non-linear MEs. Note, that in our codes for ME calculation based on the recurrence relationships (20) and (21) all nonlinear MEs $K_{r_{1}, l, r-r_{1}, 0}^{r, l}$ are necessarily calculated. The calculation of $\bar{\Lambda}_{r, r_{1}, l}\left(T_{1}\right)$ via formula (54) is essentially simpler and gives an opportunity to advance further than those using a direct numerical integration. For any Maxwellian molecules, an expression (54) is simplified as the MEs are non zero only if $k=r-r_{1}$ :

$$
\bar{\Lambda}_{r, r_{1}, l}\left(T_{1}\right)=\left(1-\frac{T_{0}}{T_{1}}\right)^{r-r_{1}} K_{r_{1}, l, r-r_{1}, 0}^{r, l}\left(T_{1}\right) .
$$

The easier method is used in the previous section. There is no need to solve a system of moment equations in the new basis to attenuate the advantages of such a method. Building up the moments in initial basis $C_{r l}^{0}$, one can perform them in the new basis and find $C_{r l}^{1}$.

Consider an example: $\in=1, t=5$. The moments $C_{r l}$ in a basis $T_{0}$ are very large at such field and time. A code for transformation of the moments built up in a basis $T_{0}$ (Bas0) to a basis $T_{1}$ (Bas1) was created.

Fig. (20) presents the calculation results at $R T=1.5$. One can see that in a subspace $l=0$ a transfer to a new basis is accompanied by a change in maximum value $C_{r l}$ by $\sim 5$ orders of magnitude and in subspace $l=10-$ by $\sim 3$ orders. In both subspaces, a shift of distribution $C_{r l}$ occurs toward domain of small $r$ - a coordinate of maximum decreases drastically (e.g., at $l=0$, it changes from 24 to 9 ).

Thus, even a comparatively small change in basis temperature results in the very strong fall in maximum on a dependence $C_{r l}$ on $r$, the latter resulting in facilitation when restoring the DF via its moments (as it was shown above on example of Coulomb interaction). However, there is no any success in an increase of the electric field and convergence range of the DF.

\subsubsection{DF Expansion in Terms of Spherical Harmonics}

When transferring to the moment representation of the Boltzmann equation at expansion in terms of the spherical 

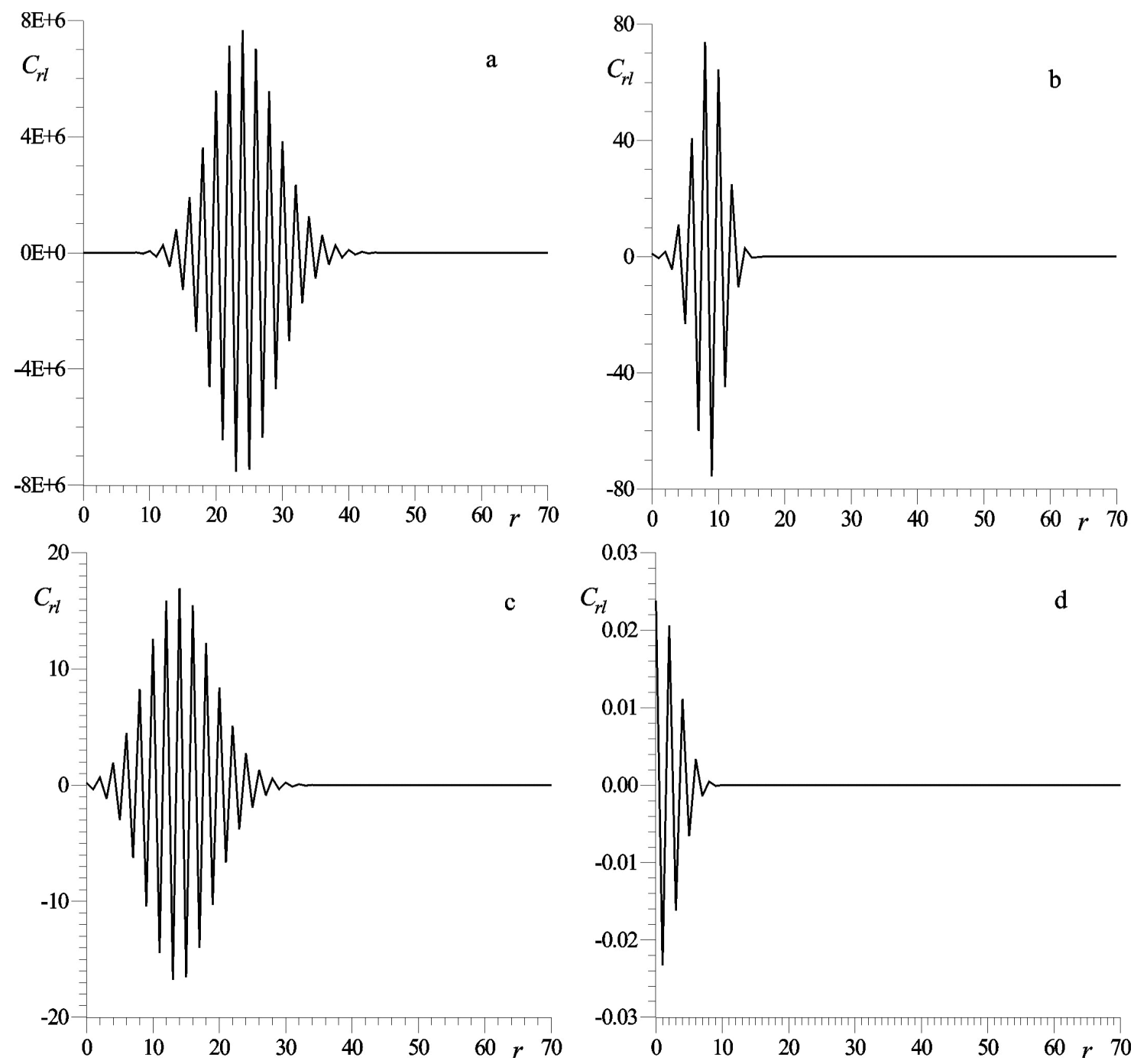

Fig. (20). Dependence of the moments $C_{r l}$ on $r$ for CEM-model in two bases with temperatures $T_{0}$ and $T_{1} ; R T=1.5, L_{0}=37, R_{0}=70$. (a) Bas0, $l=0,(\mathbf{b})-$ Bas1, $l=\mathrm{o},(\mathbf{c})-\mathrm{Bas} 0, l=10,(\mathbf{d})-\mathrm{Bas} 1, l=10$.

Hermite polynomials, one can dwell on the stage of expansion in terms of the spherical harmonics not involving the Sonine polynomials. There are no constraints on the Grad criterion with such expansion, and the DF is represented as follows

$$
f(\mathbf{c})=\sum_{l=0}^{\infty} \sum_{m=0}^{l} \sum_{i=0}^{1} f_{l, m}^{i}(c) Y_{l, m}^{i}(\Theta, \varphi)
$$

It is shown in [3] that, the collision integral in the most general (non-linear) case, can be given as follows

$$
J(\mathbf{c})=\sum_{l, m, i} Y_{l, m}^{i}(\Theta, \varphi) J_{l, m}^{i}(c)
$$

and

$$
\begin{aligned}
& J_{l, m}^{i}(c)= \\
& \sum_{l_{1}, m_{1}, i_{1}, l_{2}, m_{2}, i_{2}} \int_{0}^{\infty} \int_{0}^{\infty} G_{l_{1}, m_{1}, i_{1}, l_{2}, m_{2}, i_{2}}^{l, m, i}\left(c, c_{1}, c_{2}\right) f_{l_{1}, m_{1}}^{i_{1}}\left(c_{1}\right) f_{l_{2}, m_{2}}^{i_{2}}\left(c_{2}\right) d c_{1} d c_{2}
\end{aligned}
$$

Non-linear kernels $G_{l_{1}, m_{1}, i_{1}, l_{2}, m_{2}, i_{2}}^{l, m,}$ can be non zero only if $\mid l_{l}$ $-l_{2} \mid \leq l \leq l_{1}+l_{2}, l+l_{1}+l_{2}$ is the even number (generalized
Hecke theorem), $m=\left|m_{1} \pm m_{2}\right|$ and $i+i_{1}+i_{2}$ is the even number. It is also shown that

$G_{l_{1}, m_{1}, i_{1}, l_{2}, m_{2}, i_{2}}^{l, m, i}\left(c, c_{1}, c_{2}\right)=\breve{Z}_{m_{1}, i_{1}, m_{2}, i_{2}}^{m, i}\left(l, l_{1}, l_{2}\right) G_{l_{1}, l_{2}}^{l}\left(c, c_{1}, c_{2}\right)$,

where $\breve{Z}_{m_{1}, i_{1}, m_{2}, i_{2}}^{m, i}\left(l, l_{1}, l_{2}\right)$ are numerical coefficients which are easily expressed via the Klebsh-Gordon coefficients [3]. Each kernel $G_{l_{1}, l_{2}}^{l}\left(c, c_{1}, c_{2}\right)$ is represented as

$$
G_{l_{1}, l_{2}}^{l}\left(c, c_{1}, c_{2}\right)=G_{l_{1}, l_{2}}^{(+) l}\left(c, c_{1}, c_{2}\right)-G_{l_{1}, l_{2}}^{(-) l}\left(c, c_{1}, c_{2}\right)
$$

If (57) is generalized on the case of two-component mixture and a function on which the scattering occurs is set to be equal to the Maxwellian one, we transfer to the linear kernel. In this case we have $l_{2}=m_{2}=i_{2}=0$, the generalized Hecke theorem transforms into the common one $\left(\left(l_{1}=l, m_{1}=\right.\right.$ $\left.\left.m, i_{1}=i\right)\right)$, the numerical coefficients $\breve{Z}_{m, i, 0,0}^{m, i}(l, l, 0)=1$, and from (57), (58) we obtain

$$
J_{l, m}^{i}(c)=-k(c) f_{l, m}^{i}(c)+\int_{0}^{\infty} L_{l}^{(+)}\left(c, c_{1}\right) f_{l, m}^{i}\left(c_{1}\right) d c_{1} .
$$


Hecke [19] built up an expansion in terms of the spherical harmonics of the linearized Hilbert kernel [20] for the hard-sphere model and obtained the formulas for $L_{l}^{+}\left(c, c_{1}\right)$ and $k(c)$. In our denominations, they take a form

$$
\begin{aligned}
& k(c)=\frac{e^{-c^{2}}}{\pi^{1 / 2}}+(1 / 2 c+c) \Phi(c), \\
& L_{l}^{+}\left(c, c_{1}\right)= \\
& \quad\left(\frac{1}{\pi}\right)^{3 / 2} e^{-c^{2}} 4 \pi\left[\frac{1}{2 l+1} \frac{m i^{2 l+1}}{\left(c c_{1}\right)^{l+1}}\right. \\
& \left.\quad+\frac{1}{c c_{1}} \int_{|u|<m i} u e^{u 2} \frac{\sqrt{\pi}}{2} \Phi(u) P_{l}(u / c) P_{l}\left(u / c_{1}\right) d u\right] .
\end{aligned}
$$

Here, $\Phi(c)$ is the probability integral and $m i=\min \left(c, c_{1}\right)$. It is shown in [3] that the kernels $G_{l_{1}, l_{2}}^{l}\left(c, c_{1}, c_{2}\right)$ in (58) are expressed via MEs as follows

$$
\begin{aligned}
& G_{l_{1}, l_{2}}^{l}\left(c, c_{1}, c_{2}\right)= \\
& \quad M(c) \sum_{r} \sum_{r_{1}} \sum_{r_{2}} c^{l} S_{l+1 / 2}^{r}\left(c^{2}\right) K_{r_{1}, r_{2} l_{2}}^{r l} \frac{c_{1}^{l_{1}} S_{l_{1}+1 / 2}^{r_{1}}\left(c_{1}^{2}\right)}{\sigma_{r_{1} l_{1}}} \frac{c_{2}^{l_{2}} S_{l_{2}+1 / 2}^{r_{2}}\left(c_{2}^{2}\right)}{\sigma_{r_{2} l_{2}}}
\end{aligned}
$$

Here

$$
\sigma_{r l}=\frac{\Gamma(r+l+3 / 2)}{2 \pi^{3 / 2} r !}
$$

Formulas (63) give a possibility to calculate the kernels $G_{l_{1}, l_{2}}^{l}\left(c, c_{1}, c_{2}\right)$ for arbitrary interaction law in the linear $\left(l_{2}=\right.$ $0, l_{1}=l$ or $l_{1}=0, l_{2}=l$ ) and non-linear cases, naturally, if the MEs with large indices are known. Remind that the recurrence relationships, with which use the MEs are built up, valid both as for the MEs of full collision integral and as for the MEs of gain and loss terms separately. One can easily see that the linear gain kernel $L_{l}^{+}\left(c, c_{1}\right)$ is represented as

$$
L_{l}^{+}\left(c, c_{1}\right)=M(c) \sum_{r=0}^{\infty} \sum_{r_{1}=0}^{\infty} \frac{1}{\sigma_{r_{1} l}} c^{l} S_{l+1 / 2}^{r}\left(c^{2}\right) K_{r_{1}, 00}^{r l+} c_{1}^{l} S_{l+1 / 2}^{r_{1}}\left(c_{1}^{2}\right) .
$$

Illustrate the advantages of the DF calculation when expanding only in terms of harmonics with the hard-sphere model in the case of a weak field. As mentioned above, when calculating the Chapman-Enskog correction $\varphi(\mathbf{c})$ using the non-stationary moment method, the progress up to $c=9$, 8 (even taking into consideration 250 expansion terms of the Sonine polynomials) can be successfully obtained, a tendency to saturation for the stationary DF being exposed (see Fig. (6)). At small $\in$, DF is $f(\mathrm{c})=M(c)(1+$ $\in \varphi_{1}(c) P_{1}(\cos (\Theta))$, and, in the spatially uniform case, the Boltzmann equation is transformed to equation for $\varphi_{1}(c)$ as follows

$$
\frac{\partial \varphi_{1}(c, t)}{\partial t}-2 c=\int_{0}^{\infty} \tilde{L}_{1}^{+}\left(c, c_{1}\right) \varphi_{1}\left(c_{1}, t\right) c_{1}^{2} d c_{1}-k(c) \varphi_{1}(c, t),
$$

where

$$
\tilde{L}_{1}^{+}\left(c, c_{1}\right)=\frac{M\left(c_{1}\right)}{M(c)} L_{1}^{+}\left(c, c_{1}\right)
$$

The stationary equation (65) takes a form

$k(c) \varphi_{1}(c, \infty)-\int_{0}^{\infty} \tilde{L}_{1}^{+}\left(c, c_{1}\right) \varphi_{1}\left(c_{1}, \infty\right) c_{1}^{2} d c_{1}=2 c$.

Our analytical studies on the equation (66) at $c \rightarrow \infty$ show that, for the hard-sphere model, when $k(c)$ and $L_{1}^{+}\left(c, c_{1}\right)$ is determined via the formulas (61) and (62) in the standard normalization

$$
\varphi_{1}(\mathrm{c}, \infty)=6 .
$$

Non-stationary equation (65) is solved by the $4^{\text {th }}$ order Runge-Kutta method as well as the moment system (35). Preliminary, the matrix representation of Hecke kernel (62) is calculated in velocity points $c$ and $c_{1}$. The calculation results are presented in Fig. (21). As it is seen in Fig. (21), an approach using the kernel gives an increase in the DF convergence range by one order of magnitude.

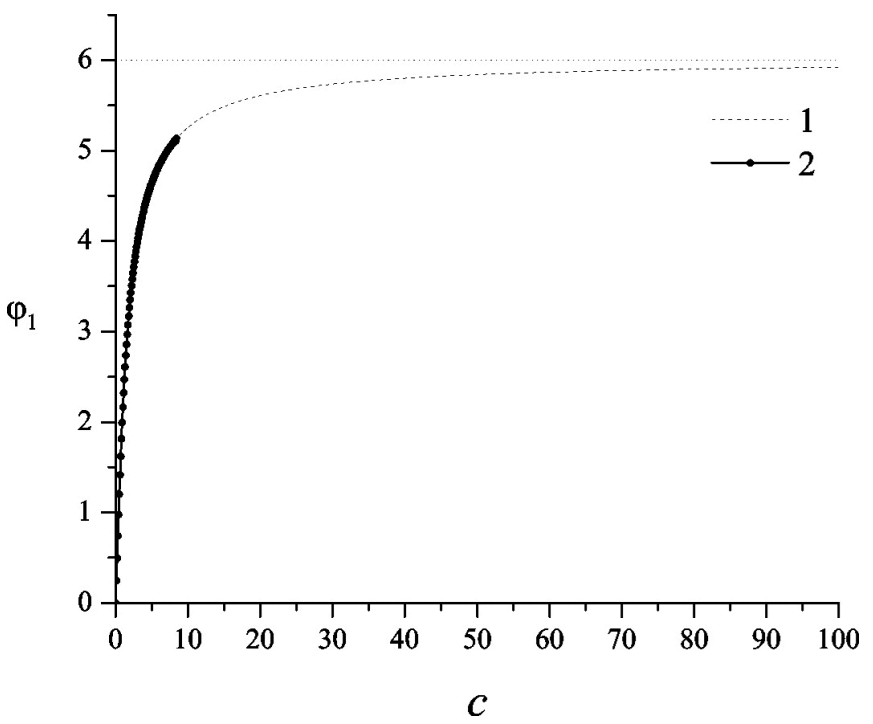

Fig. (21). Stationary Chapman-Enskog function $\varphi_{1}(c)$ for the hardsphere model. 1 - a solution of equation (65) with Hecke kernel (62), 2 - a solution with the moment system (35).

An expression (64) gives a principal possibility to build up the linear kernels for arbitrary interaction potential. However, direct calculation via a formula (64) leads to the problems related with the series cut-off. Such problem was considered earlier in [30] and [31] for the linear kernels in the case of the pseudo-Maxwellian molecules and hard spheres using the asymptotic expansions. This approach gives opportunity to calculate the kernels with a high accuracy, the latter being demonstrated by a comparison with analytical Hecke expression (62). Concluding this section, we note that when considering diffusion in a neutral gas (with no electric field), the Chapman-Enskog correction function $\varphi_{d}$ is related with $\varphi_{1}$ via equality

$\varphi_{d}(c, t)=-\frac{1}{2} \varphi_{1}(c, t)$.

Hence, we obtain the Einstein relation between the diffusion coefficient and mobility (3) at $t \rightarrow \infty$. Moreover, it signifies that, solving the spatially uniform problem in small electric field, one can simultaneously solve the diffusion problem. The function $\varphi_{d}(c, \infty)$ was built up in [32] earlier. The stationary solution was built up via solution of the 

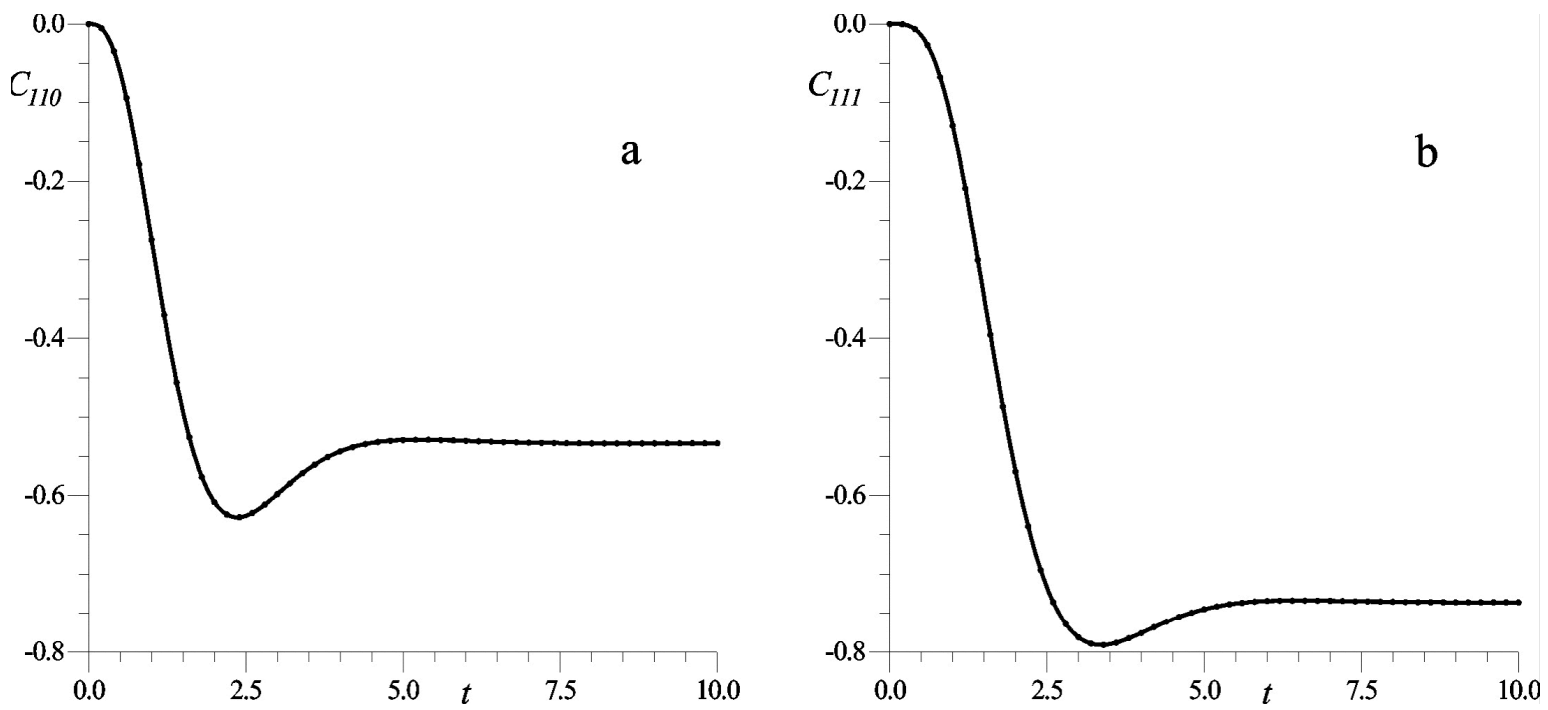

Fig. (22). Dependences of the moments $C_{110}$ and $C_{111}$ on a time for pseudo-Maxwellian molecules, $\in=1, h=1$. Solid curves are the calculations via formulas obtained using the Laplace transform; the points refer to a numerical solution of a system of moment equations. (a) $-C_{110},(\mathbf{b})-C_{111}$.

integral equation. The calculations in [32] were accomplished up to $c=6$. A ratio of our stationary solution to that in [32] is built up. Our calculations coincide with accuracy up to 4th significant digit (this accuracy being claimed in [32]).

\section{Switch-On of Crossed Electric and Magnetic Fields}

\subsection{Statement of the Problem}

The Boltzmann equation with electric field $\mathbf{E}$ directed along $z$-axis and magnetic field $\mathbf{H}$ directed along $y$-axis takes a form

$$
\frac{\partial f(\mathbf{v}, t)}{\partial t}+\frac{e}{m}(E \mathbf{k}+H(\mathbf{v} \times \mathbf{j})) \cdot \frac{\partial f(\mathbf{v}, t)}{\partial \mathbf{v}}=I(f(\mathbf{v}, t), M(T, v))(68
$$

In dimensionless form we have

$$
\frac{\partial f}{\partial t}-h c_{z} \cdot \frac{\partial f}{\partial c_{x}}+\left(\epsilon+h c_{x}\right) \cdot \frac{\partial f}{\partial c_{z}}=J(f) .
$$

Remind that a transfer to dimensionless electric field $\in$ is shown in detail in Subsubection 2.3.3. In a formula (69), time and velocity are referred to $\tau$ and $u$ where $\tau$ a mean time between collisions and $u$ is a mean thermal velocity. Dimensionless magnetic field strength is determined as follows

$$
h=\frac{e}{m} H \tau=\omega_{L} \tau
$$

Here, $\omega_{L}$ is the Larmor frequency.

On the left side of dimensionless Boltzmann equation (69), the terms related with the magnetic field are proportional to $h$ and involved in a combinations:

$$
c_{x} \cdot \frac{\partial f}{\partial c_{z}}-c_{z} \cdot \frac{\partial f}{\partial c_{x}}
$$

After very cumbersome operations, an Hermite polynomial expansion (70) obtained is as follows

$$
\begin{array}{r}
c_{x} \cdot \frac{\partial f}{\partial c_{z}}-c_{z} \cdot \frac{\partial f}{\partial c_{x}}=\frac{1}{2} M(\alpha, c) \sum C_{r l m}^{i}(t)((l-m+1) \\
(l+m) H_{r l m-1}^{i}-\left(1+\delta_{m 0}\right) H_{r l m+1}^{i}
\end{array}
$$

A contribution in moment equations due to magnetic field can be found when multiplying (71) by $h H_{r^{\prime} l^{\prime} m^{\prime}}^{i^{\prime}} / g_{r^{\prime} l^{\prime} m^{\prime}}^{i^{\prime}}$, integrating over velocity space and then omitting the primes of indices. As a result, an equation (68) corresponds to a system of moment equations

$\left.\frac{d C_{r l m}^{i}}{d t}+\frac{h}{2}(l-m)(l+m+1) C_{r l m+1}^{i}-\left(1+\delta_{m-10}\right) C_{r l m-1}^{i}\right)+$

$\in\left(\frac{2}{2 l+3} r(l+m+1) C_{r-1 l+1 m}^{i}-\frac{2}{2 l-2}(l-m) C_{r l-1 m}^{i}\right)=\sum_{r_{1}} \Lambda_{r, r_{1} l r_{r_{l} l m}^{i}}$.

An index $i=0$ refers to a spherical harmonics $P_{l}^{m} \cos$ $m \varphi$ and $i=1-P_{l}^{m} \sin m \varphi$. From a system (72), it follows that an index $m$ changes due to magnetic field but an index $i$ does not change. So, when considering the symmetric initial conditions, an index $i$ can be only zero in a course of relaxation and it is omitted further. In the case of Maxwellian molecules model with arbitrary dependence of angular part of scattering cross-section, a system (72) splits into subsystems because of diagonality of the interaction matrix. As a result, when using an apparatus of Lagrange transforms, the analytical solutions for the moments can be obtained. In addition, a system (72) in the most general case, is solved numerically via the Runge-Kutta method in the same manner as in the previous Sections with no magnetic field. The results of calculation of the moments $C_{110}$ and $C_{111}$ for pseudo-Maxwellian molecules are shown in Fig. (22). The analytical and numerical results coincide with 6-digit accuracy. It enables to claim the very high accuracy of numerical solution of a system of moment equations resulting in an opportunity to obtain the precise solutions in those cases when the interaction matrix is non-diagonal as well. For the models with collision frequency depended on 
velocity, as a rule, it is used non-standard normalization described above. Remind that in this normalization, at $h=0$ and $\in \rightarrow 0$, mobility equals unity.

\subsection{Reduced Physical Moments}

In the case of crossed electric and magnetic field we assign a vector of drift velocity, pressure tensor, and vector of heat flow to the physical moments. The physical moments are expressed via the mean products values of various particle velocities powers. When the DF is normalized to unity, the mean values are determined as follows

$\left\langle c_{x}^{i} c_{y}^{j} c_{z}^{k}\right\rangle=\int f(\mathbf{c}) c_{x}^{i} c_{y}^{j} c_{z}^{k} d^{3} c$.
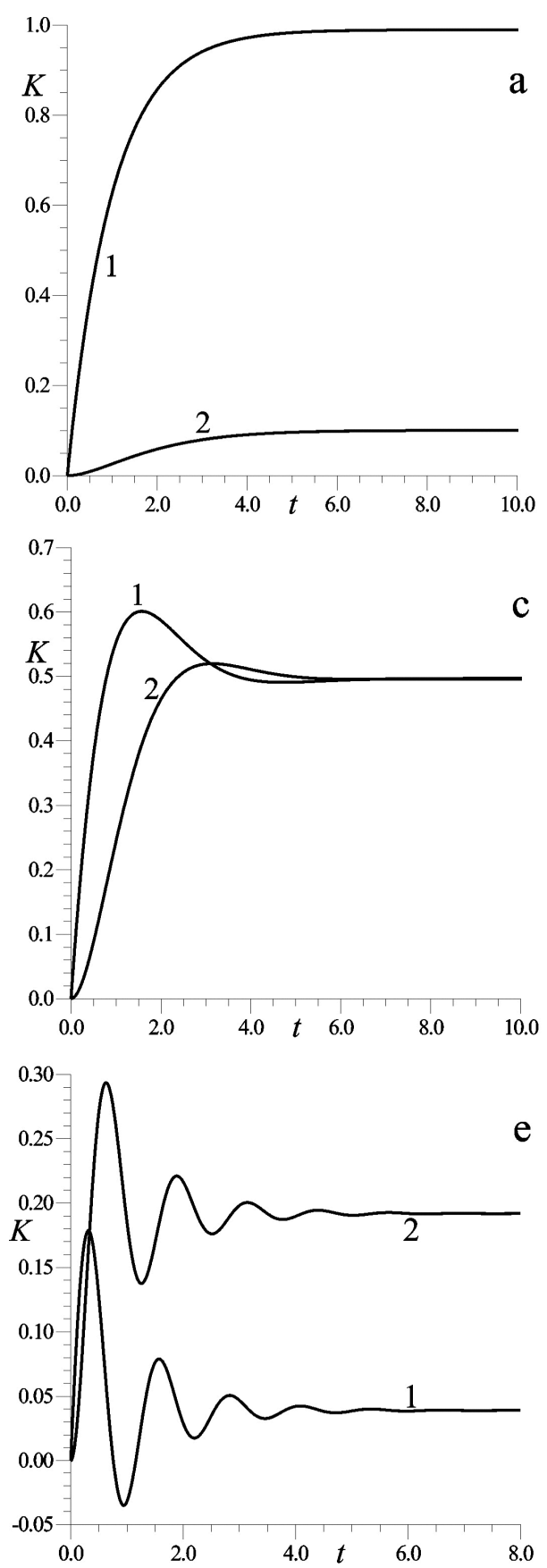

Respectively, for the mean velocity vector $\mathbf{u}_{d}$, pressure tensor $p_{\mu v}$, and vector of a heat flow $\mathbf{q}$, we have

$\mathbf{u}_{d}=\langle\mathbf{c}\rangle$,

$p_{\mu v}=\left\langle\left(c_{\mu}-u_{d \mu}\right)\left(c_{v}-u_{\mathrm{d} v}\right)\right\rangle$.

$\mathbf{q}=\left\langle\left(\mathbf{c}-\mathbf{u}_{d}\right)^{2}\left(\mathbf{c}-\mathbf{u}_{d}\right)\right\rangle$.

Analytically it is shown that, main physical moments for any value of the electric field are proportional to various powers of dimensional electrical field $\epsilon$ in the case of diagonal interaction matrix (for the models with constant collision frequency). Thus, the components of a drift velocity are proportional to $\in$, related with the field increments of diagonal components of a pressure tensor are proportional to
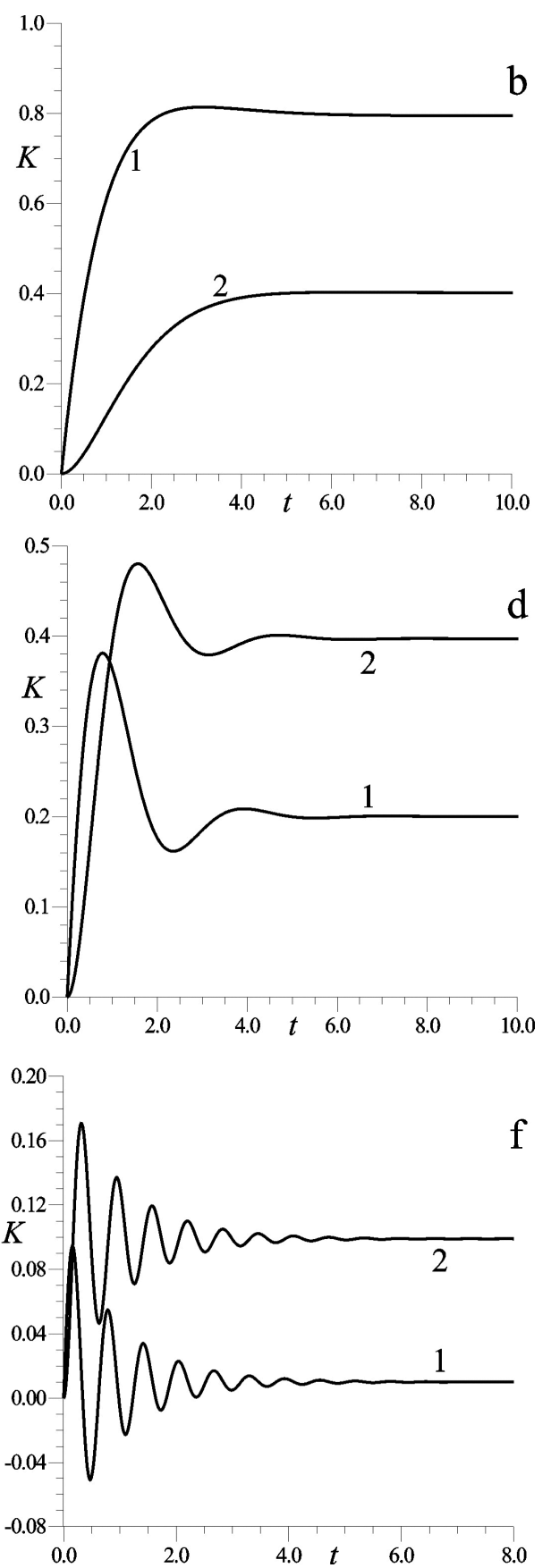

Fig. (23). Dependences of ChE- $K(t)$ (Curve1) and $K_{\text {hal }}(t)$ (Curve 2) at $\in<10^{-2}$ for HS-model at different values $h$. (a) refers to $h=.1$, (b) $h=.5,(\mathbf{c})-h=1,(\mathbf{d})-h=2,(\mathbf{e})-h=5,(\mathbf{f})-h=10$. 
$\epsilon^{2}$, and the components of a vector of heat flow are proportional to $\in^{3}$. Hence, it is of use to transfer to the reduced physical moments:

$$
\begin{gathered}
K=\frac{u_{d z}}{\epsilon}, \quad K_{h a l}=\frac{u_{d x}}{\epsilon}, \quad \bar{p}_{x x}=\frac{p_{x x}-0.5}{\epsilon^{2}}, \quad \bar{p}_{y y}=\frac{p_{y y}-0.5}{\epsilon^{2}}, \\
\bar{p}_{z z}=\frac{p_{z z}-0.5}{\epsilon^{2}}, \quad \bar{p}_{x z}=\frac{p_{x z}}{\epsilon^{2}}, \quad \overline{\mathbf{q}}=\frac{\mathbf{q}}{\epsilon^{3}} .
\end{gathered}
$$

Here, $K$ is mobility of ions along the electric field, and $K_{\text {hal }}$ is determined by us as a Hall mobility (i.e., mobility along $x$-axis directed perpendicularly to the electric and magnetic fields). The great advantage of the switching to
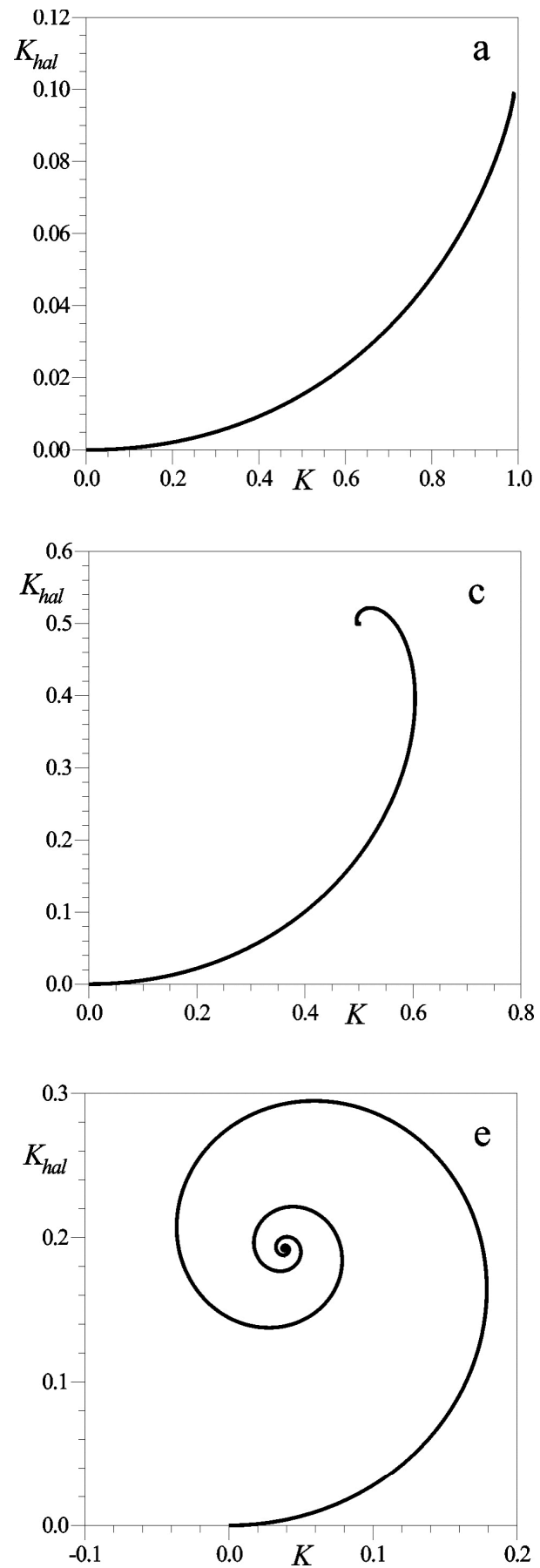

reduced physical moments is the fact that they depend on two variables ( $h$ and $t$ )only. When building up the reduced physical moments, one can easily find the physical moments themselves reversing the formulas (77) at any value of $\epsilon$.

For all other models in a domain of weak electric field, one can determine the reduced physical moments, which do not depend on $\in$ in this domain. However, our detailed numerical calculations show that, if the reduced drift velocity (mobility) and reduced pressure tensor are determined in the same manner as in the case of diagonal interaction matrix, a heat flow turns out to be proportional to $\epsilon$ but not $\epsilon^{3}$. For all models with non-diagonal interaction
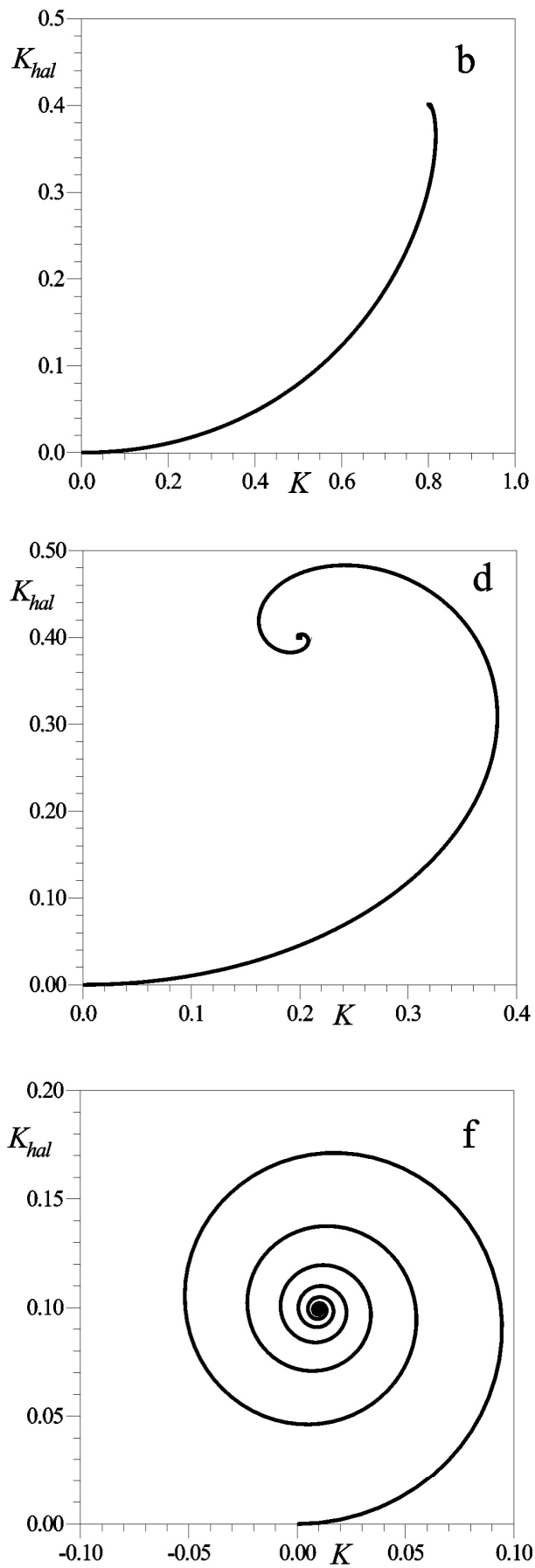

Fig. (24). Dependence of $K_{h a l}$ on $K$ at $\in<10^{-2}$ for HS-model at different values $h$. (a) refers to $h=.1,(\mathbf{b})-h=.5,(\mathbf{c})-h=1,(\mathbf{d})-h=2$, (e) $-h=5,(\mathbf{f})-h=10$. 
matrix, it is assumed to determine the reduced physical moments as follows

$$
\begin{gathered}
K=\frac{u_{d z}}{\epsilon}, \quad K_{h a l}=\frac{u_{d x}}{\epsilon}, \quad \bar{p}_{x x}=\frac{p_{x x}-0.5}{\epsilon^{2}}, \quad \bar{p}_{y y}=\frac{p_{y y}-0.5}{\epsilon^{2}}, \\
\bar{p}_{z z}=\frac{p_{z z}-0.5}{\epsilon^{2}}, \quad \bar{p}_{x z}=\frac{p_{x z}}{\epsilon^{2}}, \quad \overline{\mathbf{q}}=\frac{\mathbf{q}}{\epsilon} .
\end{gathered}
$$

\subsection{Calculation Results}

The reduced physical moments at very weak electric field $\left(\epsilon<10^{-2}\right)$ do not depend on $\in$, they are called the Chapman-Enskog physical moments (abbreviated as ChEmoments).

For several interaction models, a behaviour of ChEmoments is studied in details at different $h$ during a transient process after a sharp switch-on of the electric field.

As an example, ChE-moments for HS-model are shown in Figs. (23-27). In Fig. (23) it is seen that a Hall current
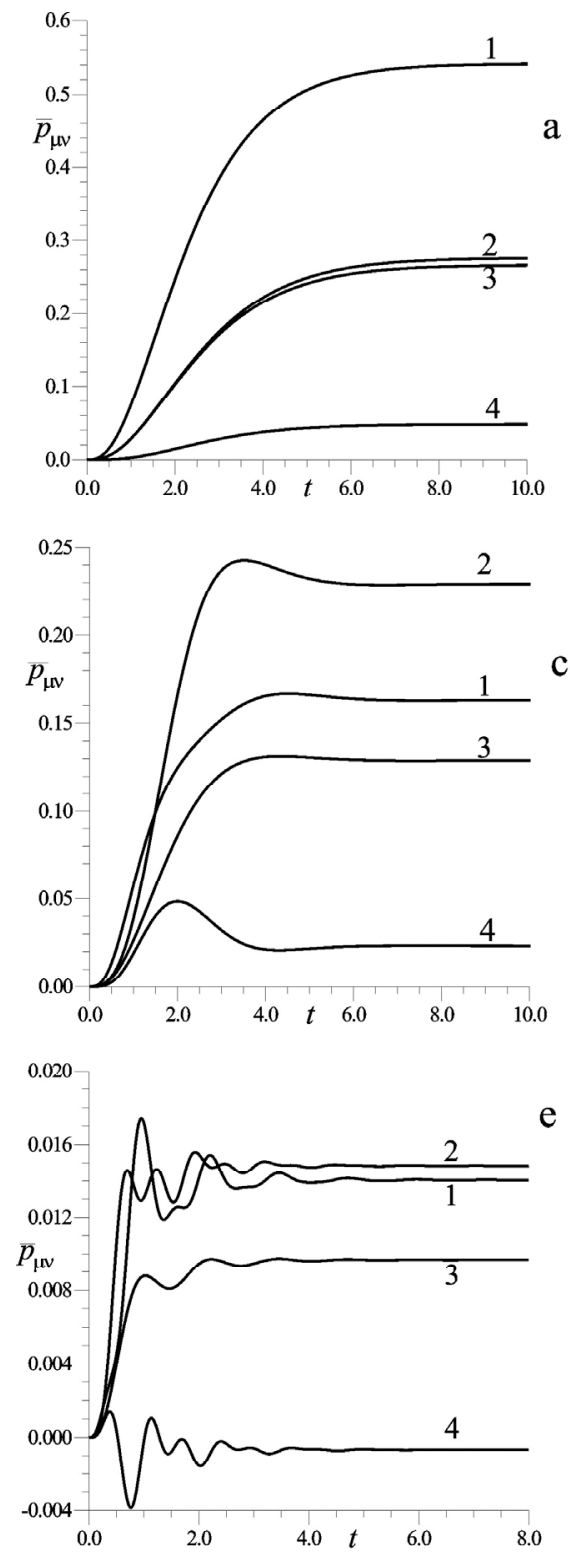

arises always with a delay relative to a current along the electric field. Oscillation amplitudes $K$ and $K_{\text {hal }}$ are very close. At large $h$, a mean value about which oscillation proceeds, for $K$ is much smaller than for $K_{\text {hal }}$. As a result, the mobility $K$ takes periodically negative values at large $h$, i.e., during certain time intervals the current along $z$-axis flows opposite to the electric field.

The behaviour of the mobility vector $\mathbf{K}$ with components $K_{\text {hal }}$ and $K$ can be imagined more clearly, if $t$ is excluded and build up a dependence $K_{\text {hal }}$ on $K$. The trajectory of a vector $\mathbf{K}$ is shown in Fig. (24). A drift velocity changes monotonously at small values of $h$. At $h \geq 0.5$, with an increase in $h$, first, a small winding arises on the trajectory, then, the new windings, and arising spiral converges on limit point corresponding to the stationary vector $\mathbf{K}$ forms. In Fig. (24), the abscissa and ordinate scales are chosen to be equal. With such a choice, absolute value of a drift velocity is proportional to a distance from coordinate origin to the point
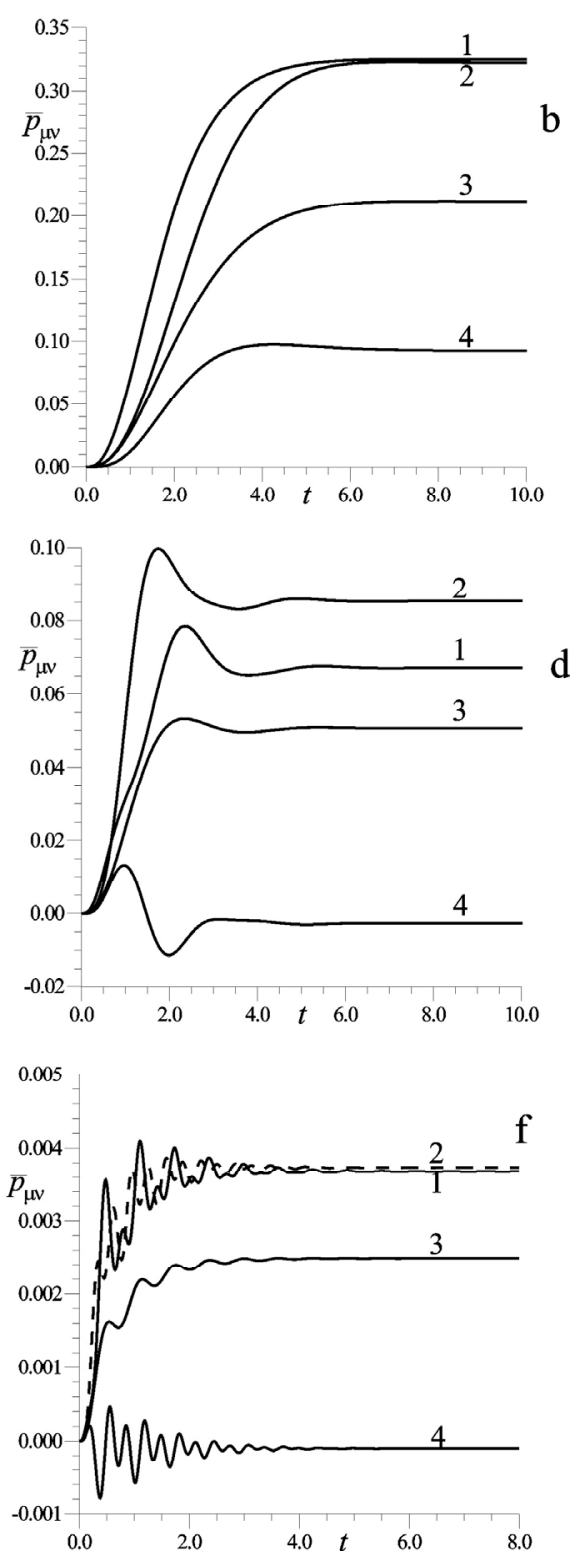

Fig. (25). Dependences of the components of Chapman-Enskog pressure tensor on time at $\in<10^{-2}$ for HS-model at different values $h$. Curves: $1-\bar{p}_{z z}, 2-\bar{p}_{x x}, 3-\bar{p}_{y y}, 4-\bar{p}_{x z}$. (a) refers to $h=.1,(\mathbf{b})-\mathrm{h}=.5,(\mathbf{c})-h=1,(\mathbf{d})-h=2,(\mathbf{e})-h=5,(\mathbf{f})-h=10$. 
under consideration with a coefficient $\in$. It can be seen in Fig. (24) that, along the developed trajectory the drift velocity moves in a circumference with decreasing radius having its center in a point corresponding to the stationary drift velocity. In Fig. (27) one can see that a vector of heat flow moves in a spiral as well as a vector of drift velocity.

The reduced physical moments change with an increase in electric field. Nevertheless, we build up and study the reduced physical moments in the domains of moderate and
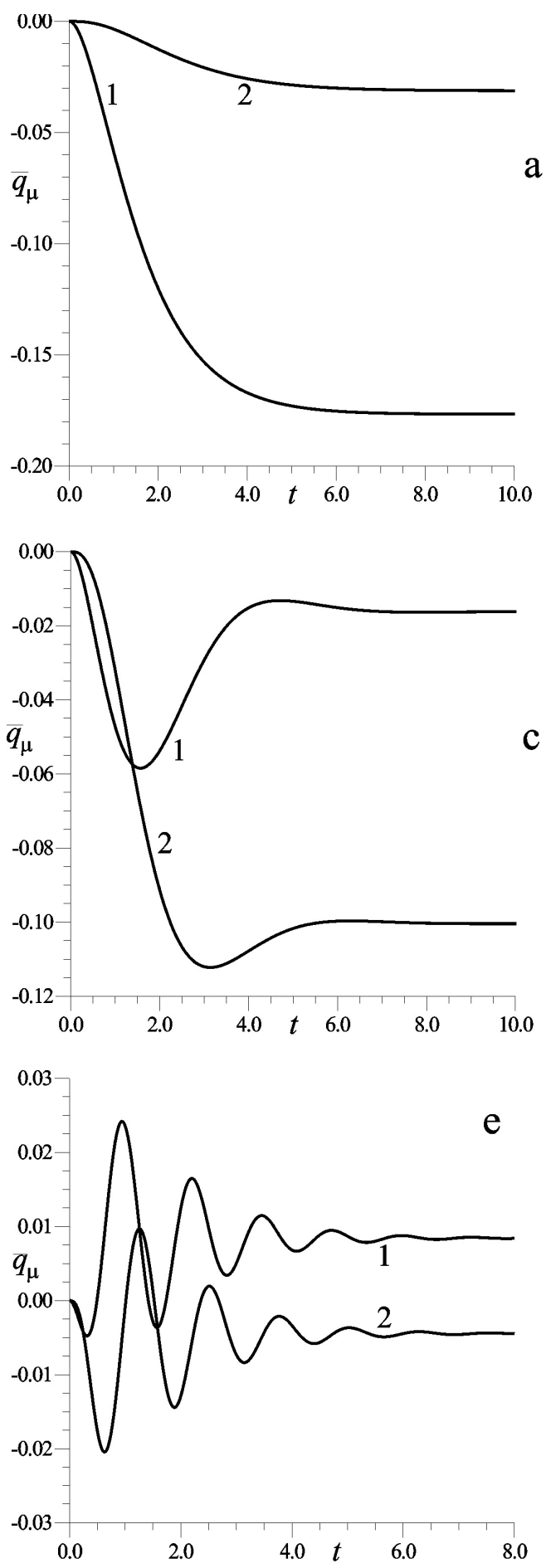

strong electric fields as well. The calculation reveals that their behaviour changes a little qualitatively with an increase in $\in$ : oscillation of the moment is observed with a Larmor frequency, and the vectors of mobility and heat flow move in a spiral.

At not so weak electric field our calculations show that at a weak magnetic field a quantitative distinction of the reduced physical moments from ChE-moments is of significance, especially, for a heat flow. However, with an
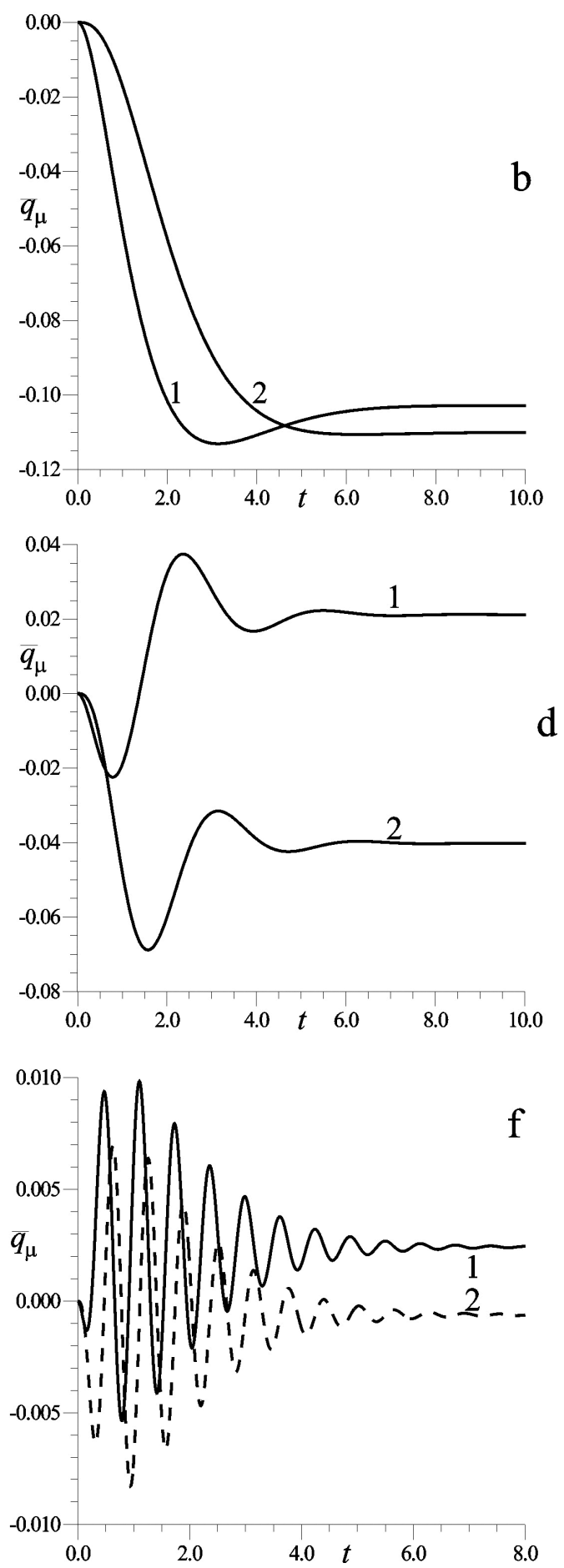

Fig. (26). Dependences of the components of Chapman-Enskog heat flow on time at $\in<10^{-2}$ for HS-model at different values $h$. Curves: $1-$ $\bar{q}_{z}, 2-\bar{q}_{x}$. (a) refers to $h=.1,(\mathbf{b}) .-\mathrm{h}=.5,(\mathbf{c})-h=1,(\mathbf{d})-h=2,(\mathbf{e})-h=5,(\mathbf{f})-h=10$. 

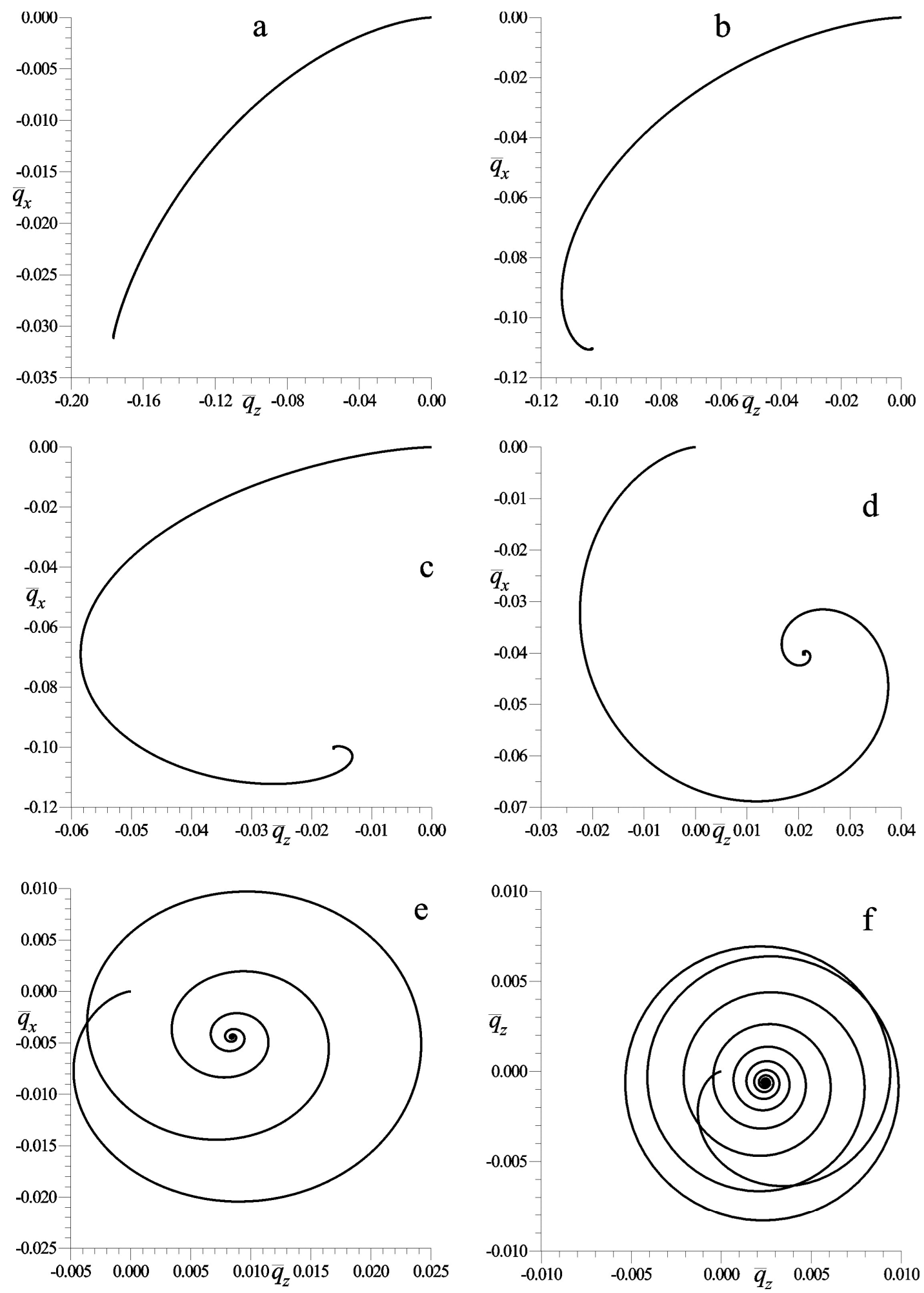

Fig. (27). Dependence $\bar{q}_{x}$ on $\bar{q}_{z}$ at $\in<10^{-2}$ for HS-model at different values $h$. (a) refers to $h=.1,(\mathbf{b})-\mathrm{h}=.5,(\mathbf{c})-h=1,(\mathbf{d})-h=2,(\mathbf{e})$ $-h=5,(\mathbf{f})-h=10$.

increase in magnetic field this distinction decreases and the reduced physical moments practically coincide with the ChE-moments at $h=5,10$.

At the weak magnetic field $(h=0.1, h=0.5)$, mobilities ( $K$ and $K_{\text {hal }}$ ) as well as the components of a pressure tensor turn out to be smaller than the corresponding ChE-moments in the case of HS-model.

\subsubsection{Runaway of Ions}

In Subsection 1.5.2, the process of ion runaway in the electric field is considered. It is shown that beginning with a value $\epsilon=0.3$ there is no stationary state. Here we investigate the influence of a magnetic field on a runaway effect. For Coulomb model the mobilities turn out to be higher than the ChE-ones, and at small $h$ and $\epsilon=0.5$ they 
tend to infinite growth. At smaller values $\in$ this effect is almost invisible but at $\in>0.5$, even more pronounced. In Figs. (28-30) a comparison in mobility behaviour at three different values $\in$ and at 6 different values $h$ is given for two models: hard spheres and Coulomb interaction. As mentioned above, this effect is due to very strong dependence of scattering cross-section on a relative velocity $\sigma \sim g^{-4}$ at the Coulomb interaction. Note, that the runaway effect can be calculated successfully via moment method. According to this aim it is necessary to use a rather large
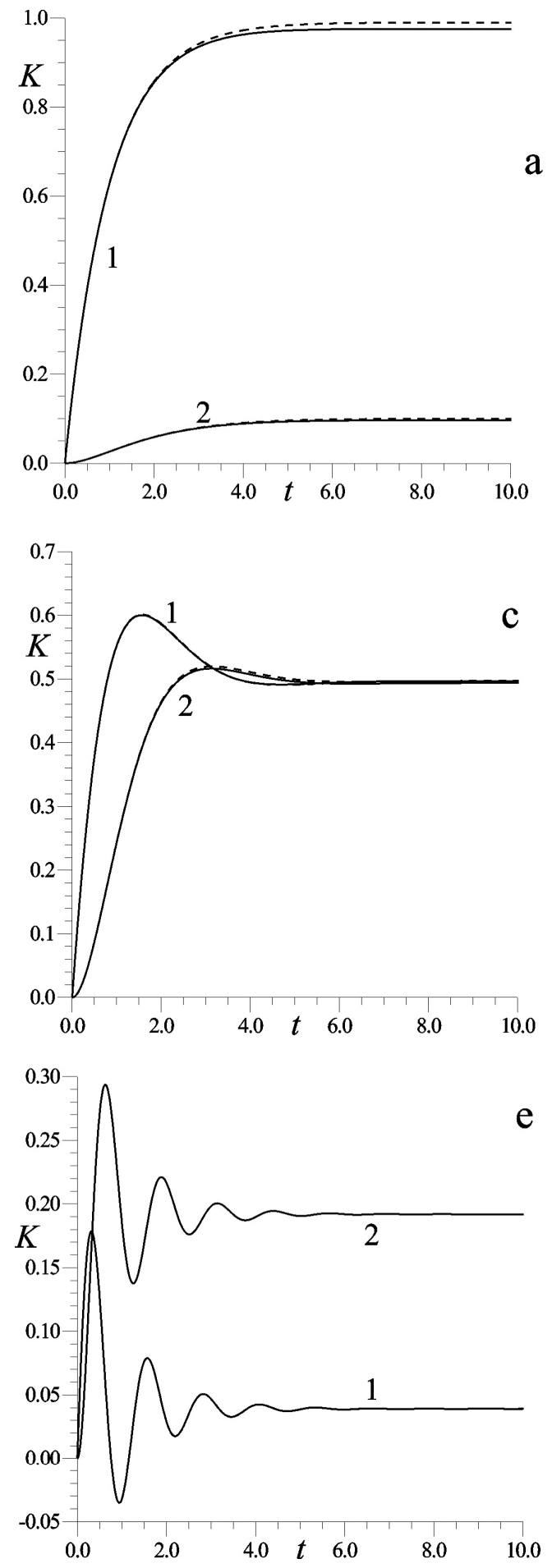

number of MEs of collision integral. But if a transient process should be calculated at of somewhat larger value $\epsilon$ (e.g., $\in=0.8$ ) when the process of ion runaway, the moment method suits well only at not so large times $(t<5)$ as well as without magnetic field. Further convergence is lost. In Figs. (28-30) it is also seen that runaway is damped with magnetic field. At the strong magnetic field $(h=5$ or $h=10)$ reduced mobility coincides with ChE-mobility, the moment method convergence being well restored.
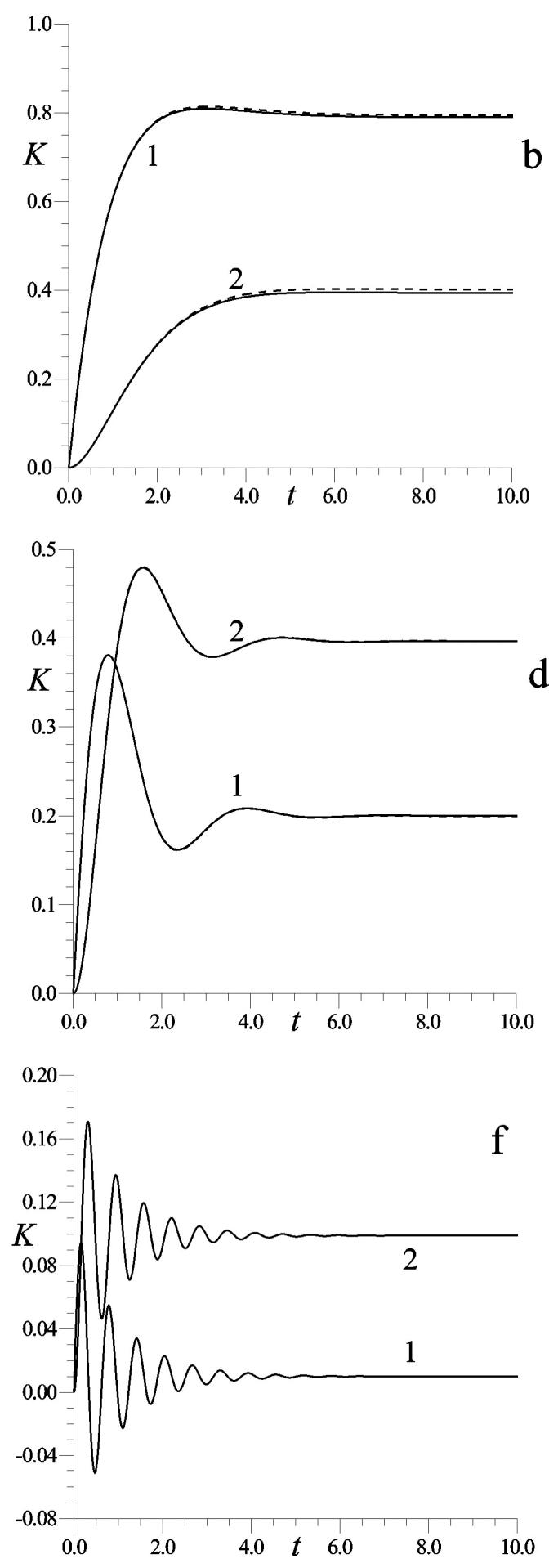

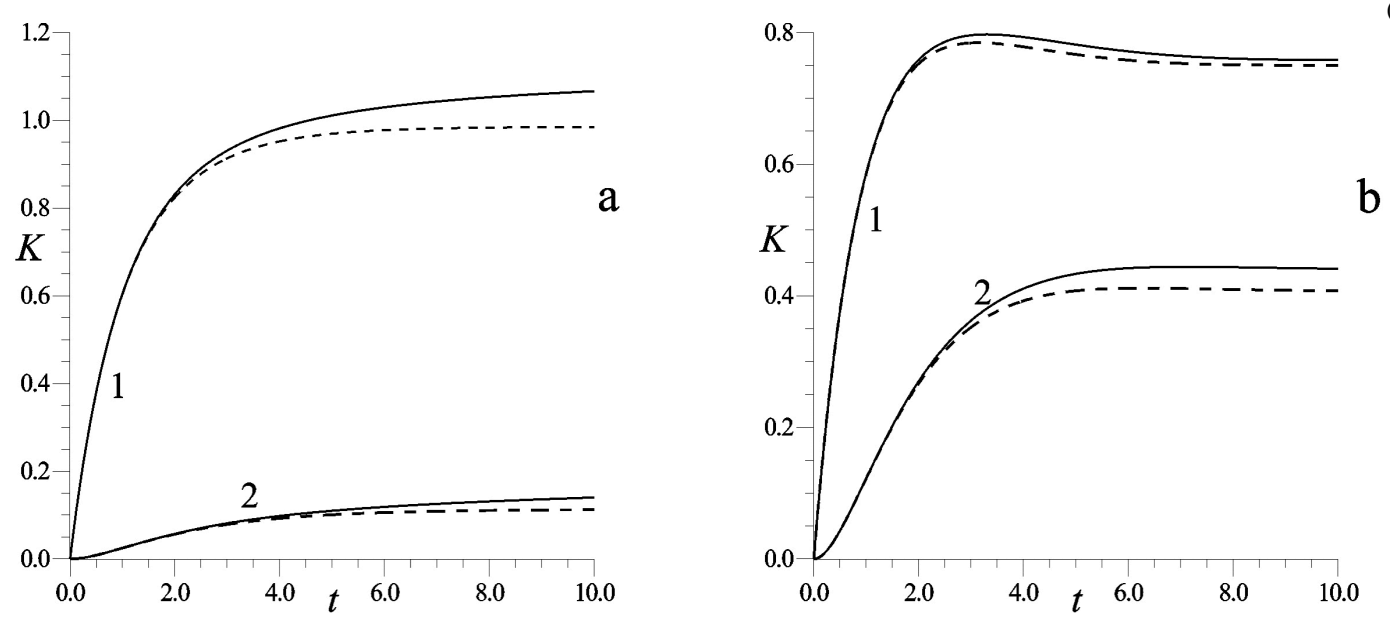

(Fig. 28). contd.....
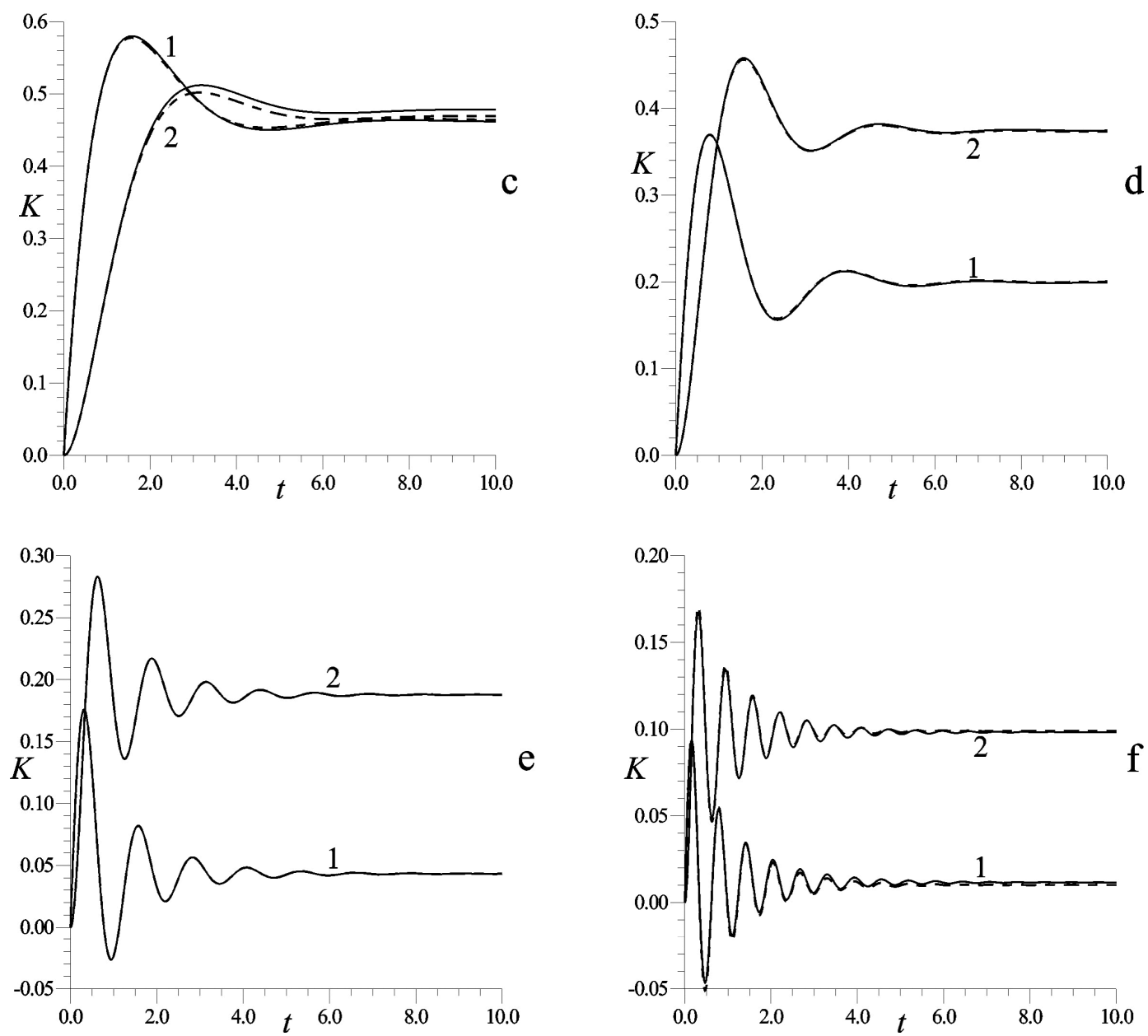

Fig. (28). Dependences $K(t)$ (Curve 1) and $K_{h a l}(t)$ (Curve 2) for HS-model (first six figures) and model of Coulomb interaction (second six figures) at $\in=0.2$ and different values $h$. Dashed curves refer to ChE-mobility. (a) $-h=.1,(\mathbf{b})-\mathrm{h}=.5,(\mathbf{c})-h=1,(\mathbf{d})-h=2,(\mathbf{e})-h=5$, (f) $-h=10$.

\subsubsection{Very Strong Electric and Magnetic Fields}

Good convergence at the strong magnetic field gives a chance to enter the domain of very strong electric fields at large $h$, i.e., that domain in which the calculations are not possible using the standard moment method without magnetic field. As an example, the results of the transient processes calculation are shown in Fig. (31), namely, mobility in the case of HS-model for $\in=10$ and $h=20$.
Note, that the transfer to the strong magnetic field needs a decrease in time step due to a high oscillation frequency at the strong magnetic field.

Analysing the calculation results for large $h(h \geq 210)$, we conclude that the departure from the ChEmoments is determined by dimensionless parameter $\kappa$

$\kappa=\frac{h}{\epsilon}$ 
For $\kappa>2$, all reduced physical moments given coincide in practice with ChE-moments. At $\kappa \sim 1.5$, the departure of mobilities from the $\mathrm{ChE}$ ones are very small, and, for higher reduced physical moments (e.g., $\overline{\mathbf{q}}$ ) the distinctions can be seen on the graph but slightly. At $\kappa=1$ the departures of mobilities from the ChE's ones, being negligible, can be seen on the graph. For higher moments the departures from ChE's can be easily seen not only in a course of the transient processes but the stationary states too.
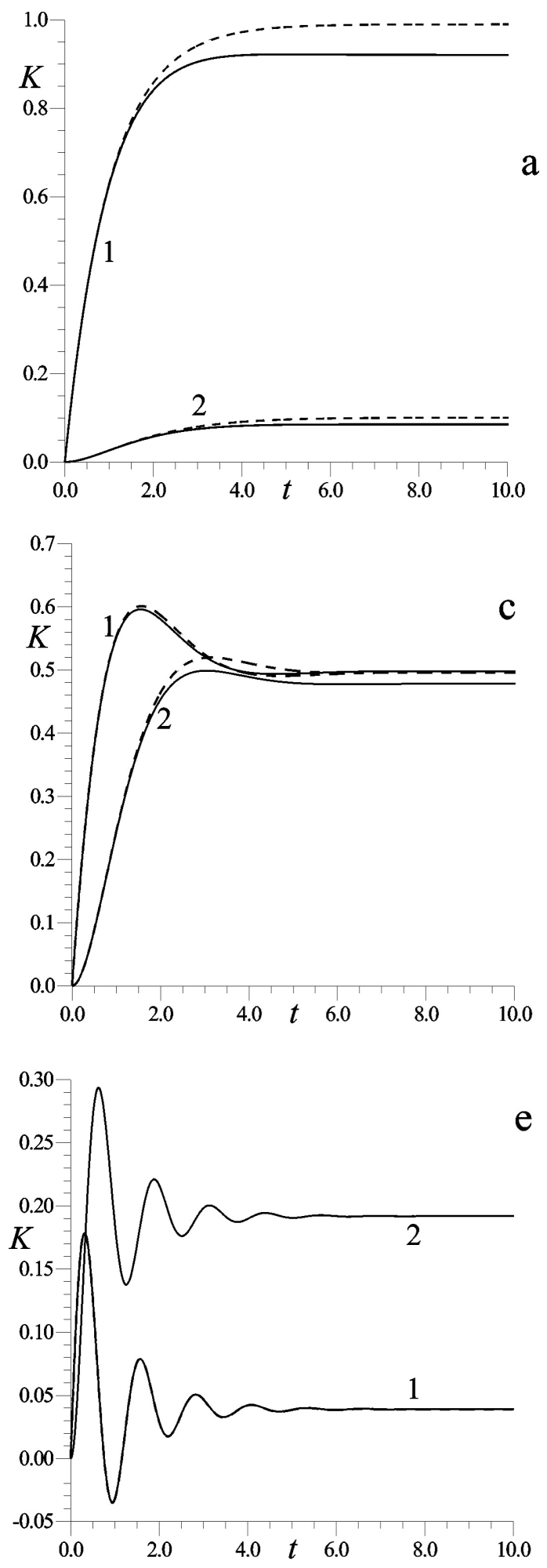

As a result of the present studies concerning the behaviour of physical moments, the conclusions can be deduced as follows:

1. If $\in$ is fixed, with an increase in $h$, Hall current reaches its maximum in $h=1$ vicinity for any interaction model. Lorentz angle determining a direction of drift velocity relative to $c_{z}$ axis (tangent of this angle equals $K_{h a l} / K$ ) approaches $45^{\circ}$ at a point $h=1$.
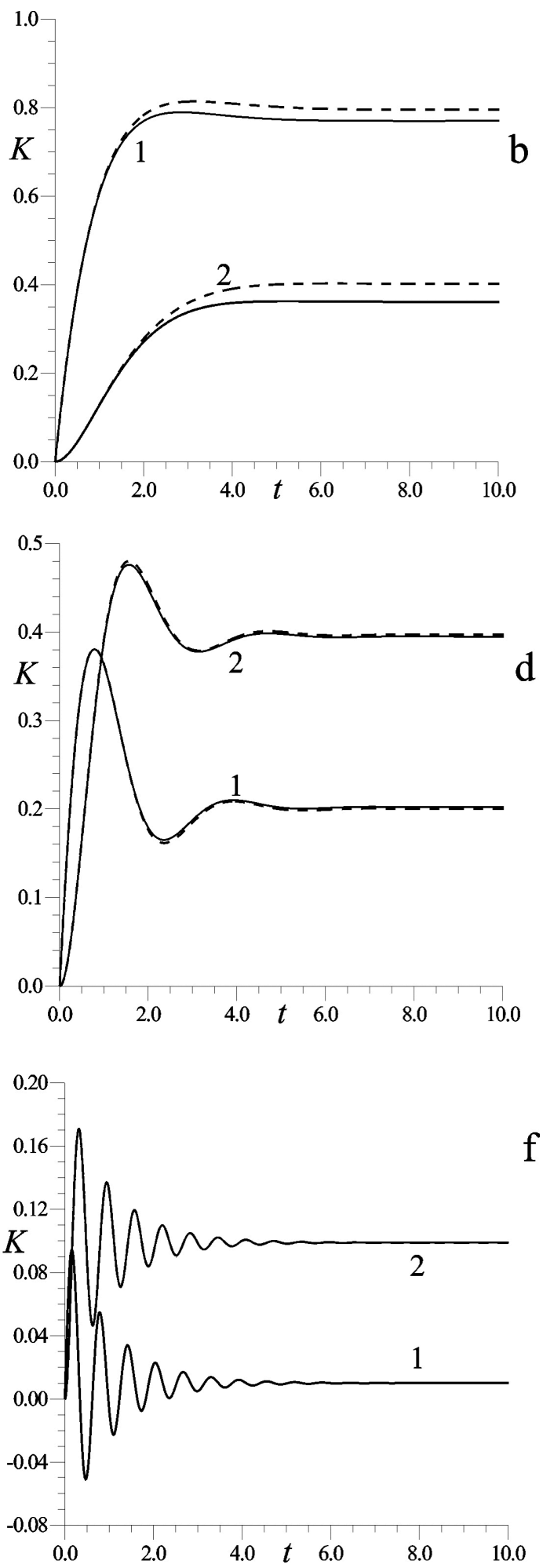

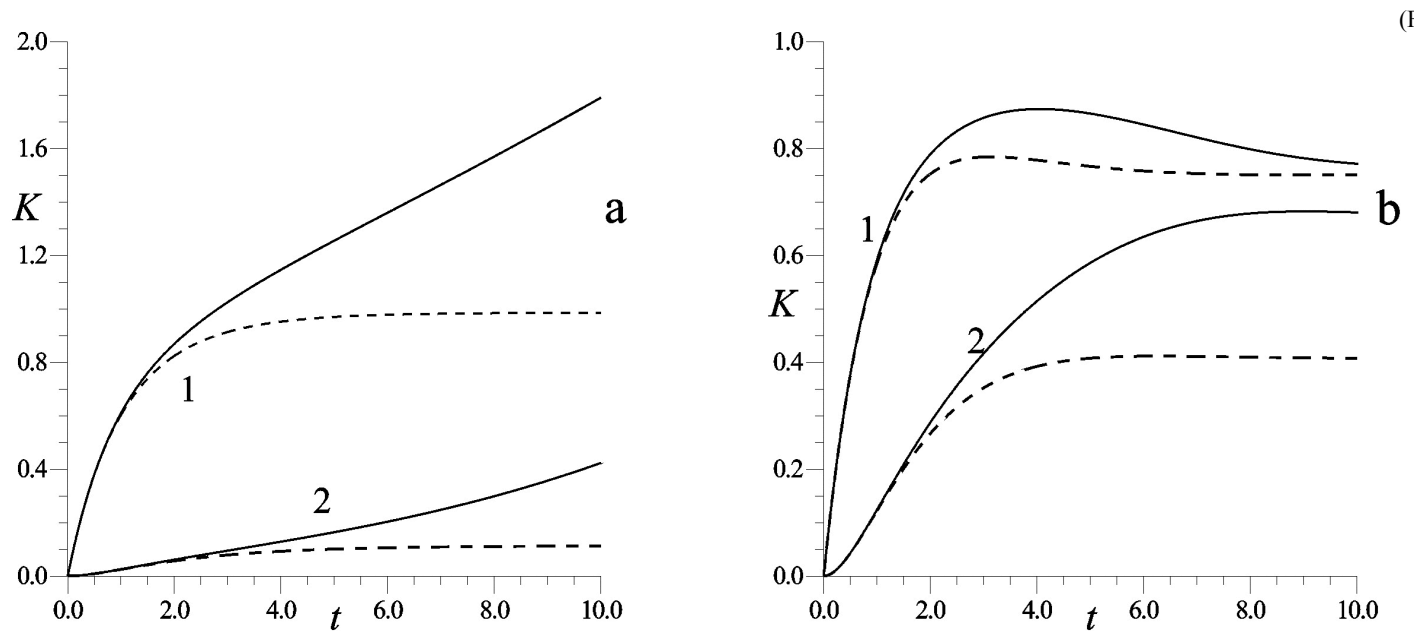

(Fig. 29). contd....
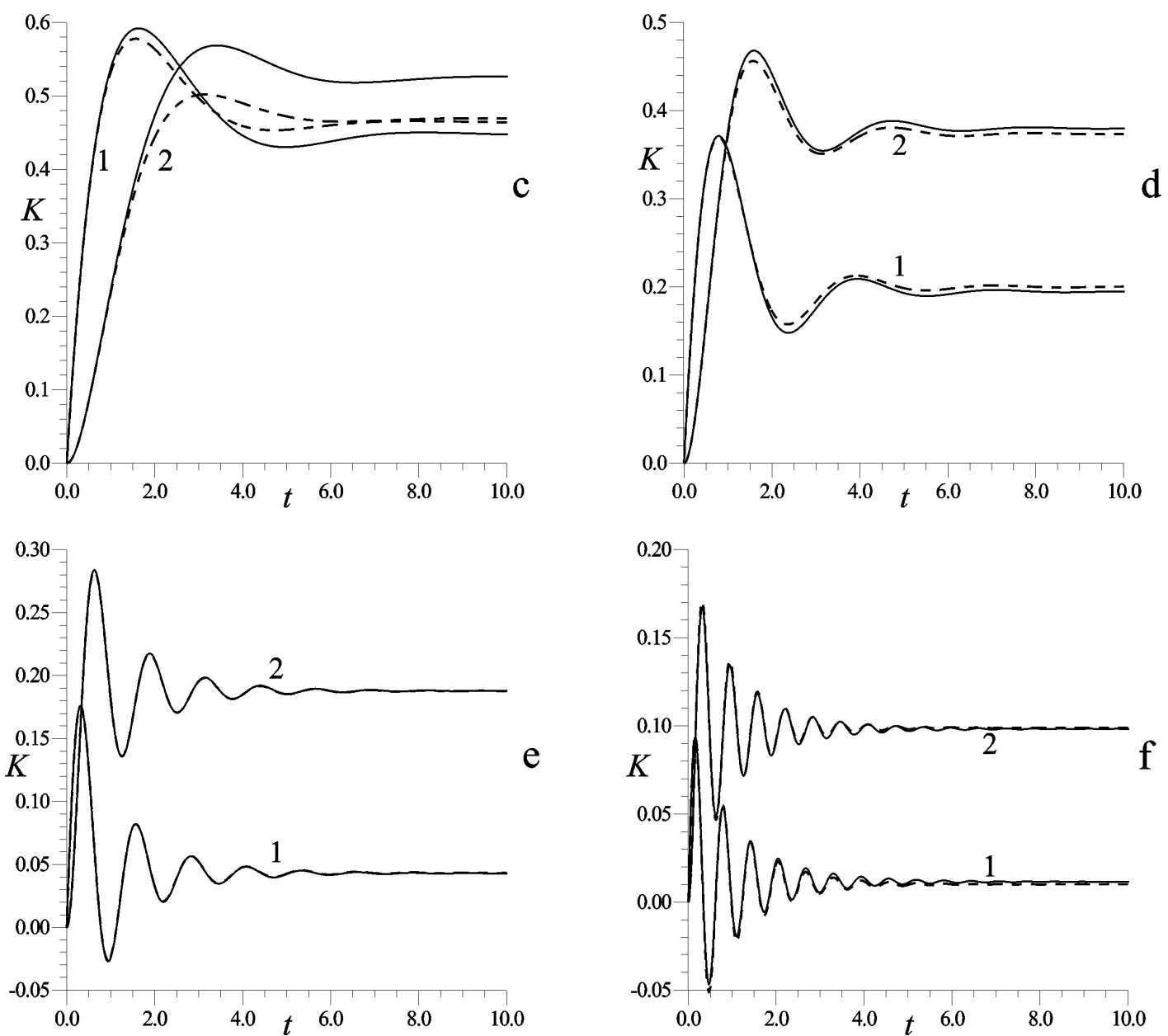

Fig. (29). Dependences $K(t)$ (Curve1) and $K_{h a l}(t)$ (Curve 2) for HS-model (first six figures) and model of Coulomb interaction (second six figures) at $\in=0.5$ and different values $h$. Dashed curves refer to ChE-mobility. (a) $-h=.1,(\mathbf{b})-\mathrm{h}=.5,(\mathbf{c})-h=1,(\mathbf{d})-h=2,(\mathbf{e})-h=5$, (f) $-h=10$.

2. At $h>1$, on temporal dependences of all physical moments the oscillations are observed and for all interaction models a drift velocity vector moves in a spiral toward the limit value.

3. In spite of a decrease in the stationary Hall current at $h>1$ its ratio to a current along $z$-axis continues to increase and the Lorentz angle tends to $90^{\circ}$ with an increase in $h$.

It is clear that an oscillation character of a behaviour of physical moments is related with a complicated and also oscillation behaviour of the DF of ions in crossed electric and magnetic fields after a instantaneous switch-on of the electric field. 


\subsection{Distribution Function}

First, consider the DF in the case of a weak electric field. The weak one, as above, we call the field at $\epsilon<10^{-2}$. Calculations show that (as with no magnetic field) at any $h$ the DF is represented as follows

$f(\mathbf{c}, t)=M(c)(1+\in \Phi(\mathbf{c}, t))$,

where $\Phi(\mathbf{c}, t)$ does not depend on $\in$. Naturally, this function depends on $h$ and, moreover, changes strongly with an
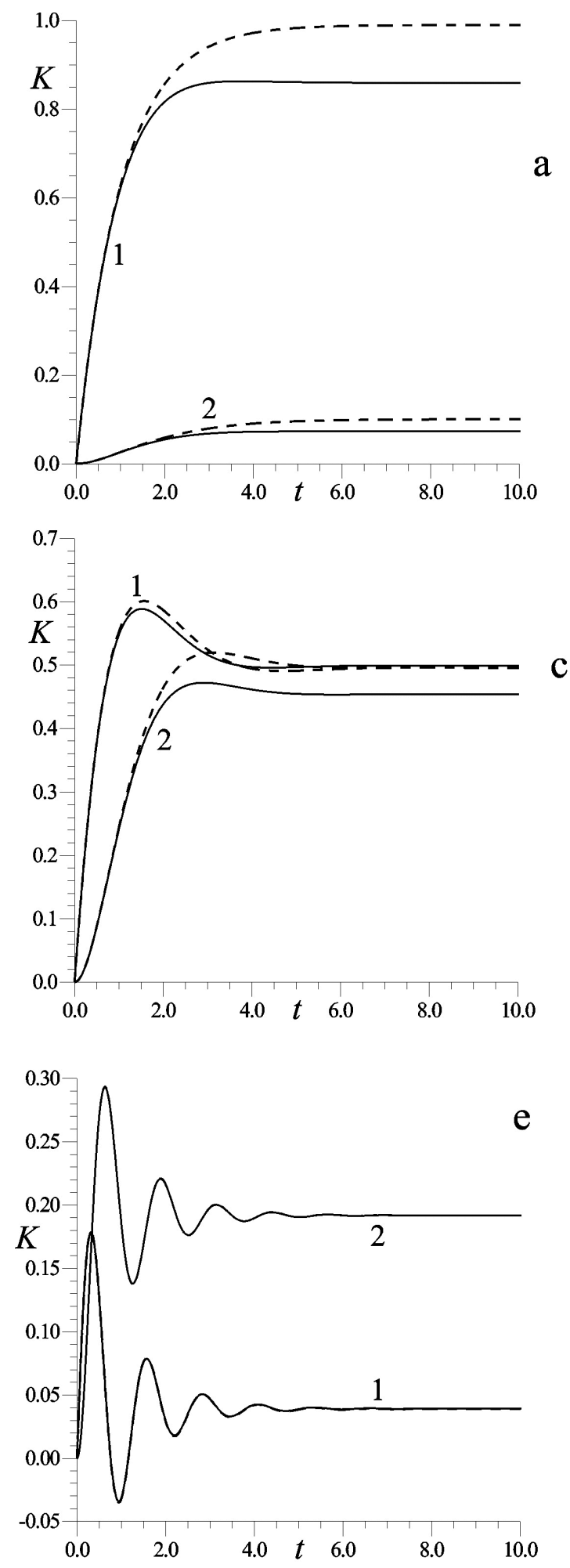

increase in $h$. Particularly, it is not axially symmetric now. With an increase in the magnetic field generally $\Phi$ rebuilding occurs in a plane $c_{x}, c_{z}$ (i.e., in a plane perpendicular to the magnetic field direction). A symmetry preserves relative to $c_{y} \rightarrow-c_{y}$ exchange. Moreover, a property takes place at fixed $c_{y}$ and $t$ as follows

$\Phi\left(-c_{x},-c_{z}\right)=-\Phi\left(c_{x}, c_{z}\right)$

Let us build up $\Phi$ at $c_{y}=0$, where $\Phi(\mathbf{c}, t)$ is being representing by a surface in $3 \mathrm{D}$ space at fixed instant. It is
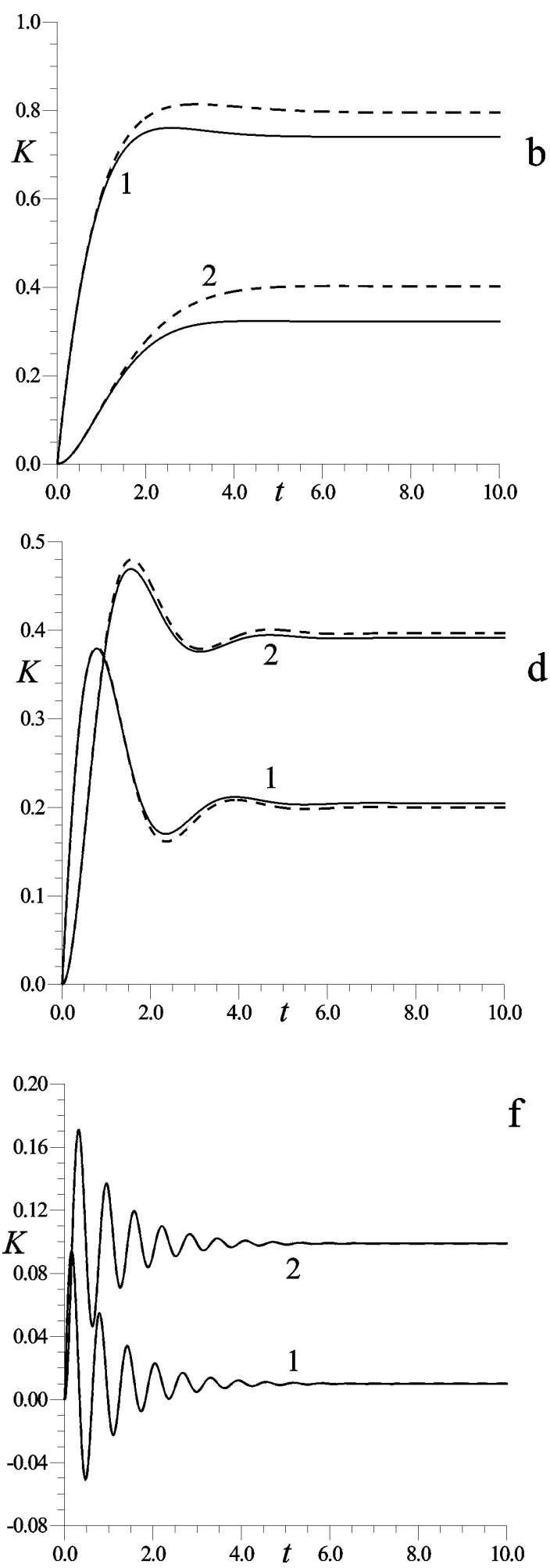

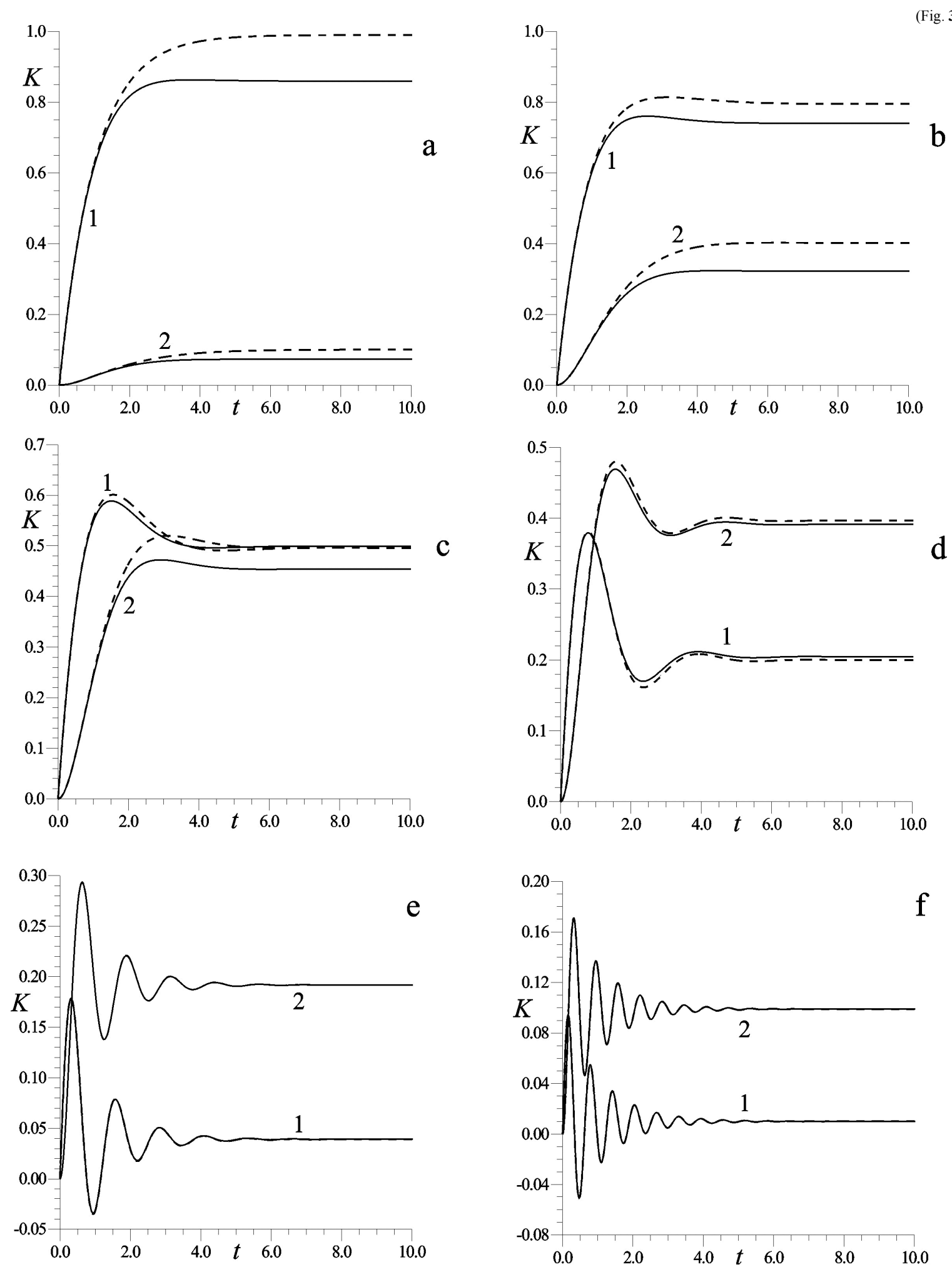

Fig. (30). Dependences $K(t)$ (Curve1) and $K_{\text {hal }}(t)$ (Curve 2) for HS-model (first six figures) and model of Coulomb interaction (second six figures) at $\in=0.8$ and different values $h$. Dashed curves refer to ChE-mobility. (a) $-h=.1,(\mathbf{b})-\mathrm{h}=.5,(\mathbf{c})-h=1,(\mathbf{d})-h=2,(\mathbf{e})-h=5$, (f) $-h=10$.

suitable to build up not a whole DF $f(\mathbf{c})$ but a correction $\Phi(\mathbf{c})$ to obtain a view on the DF at small $\in$ and emphasize DF behaviour in a domain of high velocities. It follows from calculation, that only the moments with $l=1$ contribute in $\Phi$ and the function $\Phi$ can be represented as follows

$$
\Phi(\mathbf{c})=\sum_{m=0}^{1} \Phi_{1 m}(c) Y_{1 m}(\Theta, \varphi)
$$

The functions $\Phi_{10}(c)$ and $\Phi_{11}(c)$ are built up solving a system of moment equations via summation of the corresponding moments with the Sonine polynomials. A 
polar angle $\varphi$ in $c_{x}, c_{y}$-plane is measured from a direction of $c_{x}$-axis.

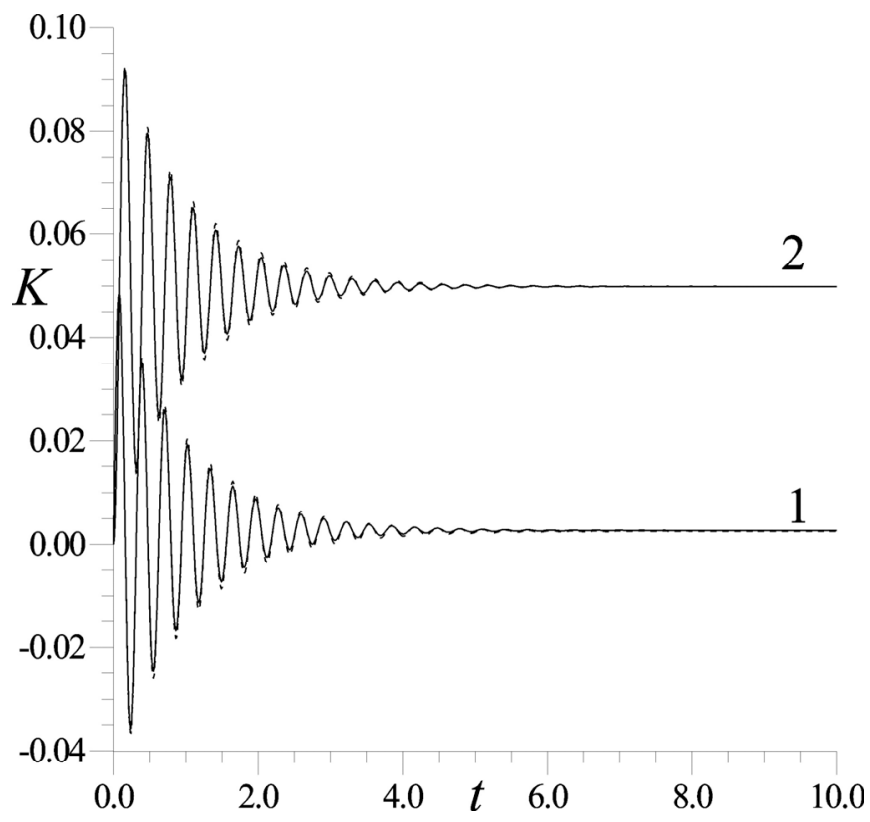

Fig. (31). Dependences $K(t)$ (Curve1) and $K_{\text {hal }}(t)$ (Curve 2) for HSmodel at $\epsilon=10$, and $h=20 ; h t=0.002, L_{0}=40, R_{0}=60$. Dashed curves (coinciding with the solid ones) refer to $\mathrm{ChE}$ mobilities.

As above (without magnetic field), introduce a rotation angle in $\left(c_{x}, c_{z}\right)$-plane which is denoted as $\psi$ (see, subsection 1.5.2). While $0 \leq \psi<\pi$, it corresponds to $\varphi=0$ and $\psi=\Theta$ in a spherical frame. For $0 \leq \psi<\pi$, we have a relationship in spherical frame $\varphi=\pi$ and $\psi=2 \pi-\Theta$. A function $\cos m \varphi$ involved in spherical harmonic takes a value $\cos \mathrm{m} \pi=(-1)^{m}$ in a domain $\psi>\pi$. For $\psi<\pi$ (from (82)) we obtain

$\Phi\left(c_{x}, c_{z}\right)=\sum_{m=0}^{1} \Phi_{1 m}(c) P_{1 m}(\Theta)$,

or

$\Phi\left(c_{x}, c_{z}\right)=\Phi_{10}(c) \cos (\Theta)+\Phi_{11}(c) \sin (\Theta)$.

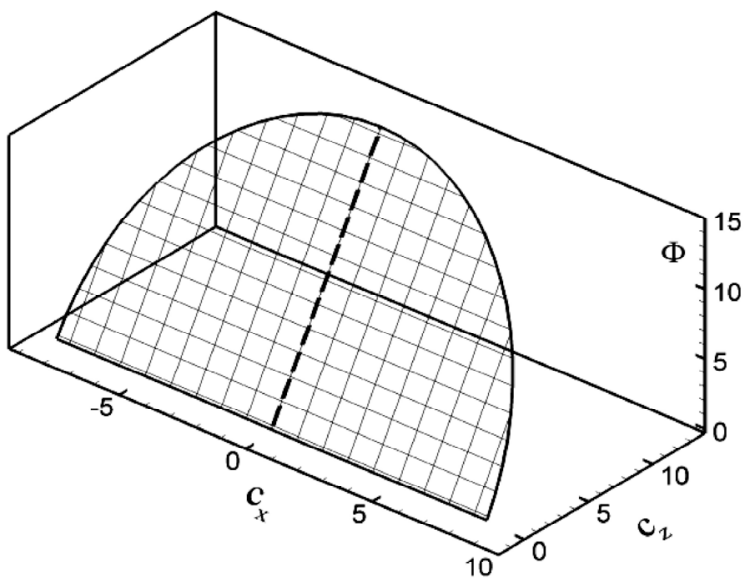

(a)
Denote

$$
R(c)=\sqrt{\left(\Phi_{10}(c)\right)^{2}+\left(\Phi_{11}(c)\right)^{2}}
$$

and determine an angle $\alpha(c)$ as follows: $\Phi_{10}(c)=R(c) \cos \alpha(c)$. Then, $\Phi_{11}(c)=R(c) \sin \alpha(c)$ and taking in consideration $\Theta=\psi$ for $\psi<\pi$ we have

$\Phi\left(c_{x}, c_{z}\right)=R(c) \cdot \cos (\psi-\alpha(c))$.

Clearly, a formula (86) is valid for the whole $0 \leq \psi<2 \pi$ plane.

From (86), it is seen that a DF $\Phi\left(c_{x}, c_{z}\right)$ is determined by two function $R(c)(85)$ and $\alpha(c)$ :

$\alpha(c)=\arctan \frac{\Phi_{11}(c)}{\Phi_{10}(c)}$.

$\mathrm{DF}$ value is $R(c)$ at fixed $c$ and if $\psi=\alpha(c)$. DF value changes strictly in cos-law when changing $\psi$. Hence, maximum on a dependence $\Phi(\psi)$ is observed at $\psi=\alpha$ and minimum is at $\psi=\alpha+\pi$. Moreover, the DF satisfies a condition (81). It gives us a possibility to be restricted to DF building-up on a semi-plane only, e.g., $c_{z} \geq 0$. A dependence $\alpha(c)$ is a projection of a line of maximums of DF on $c_{x}, c_{z^{-}}$ plane. We call $R(c)$ and $\alpha(c)$ the main characteristics of the DF. $\alpha$-line goes along $c_{z}$-axis (i.e., $\alpha(c) \equiv 0$ ) in the absence of the magnetic field. 3D figure is built up for sketch of the DF. There is a dependence $\Phi\left(c_{x}, c_{z}\right)$ on $c_{z} \geq 0$-semi plane at $c_{y}=0$. In this figure, the contours corresponding to the lines of a level $c_{x}$ and $\Phi$ are built up. In 3D picture the line of maximum is shown with thick dashed line. 3D figure is built up for the stationary state corresponding to $t \rightarrow \infty$. In addition to obtain a view on evolution process for several times the curves $R(c)$ are shown as well as the projections of $\alpha$-lines on $c_{x}, c_{z}$-plane; the latter is not built up with no magnetic field $(h=0)$.

Show the calculation results for three interaction models. In Figs. (32-35) the calculation results are shown for CEMmodel and HS-model at two values of magnetic field: $h=0$ and $h=1$. It is seen that, $\alpha$-line rotates from $c_{z}$-axis to $c_{x}$-axis during DF evolution. For CEM-model, as $\alpha$-line as $R(c)$ remain

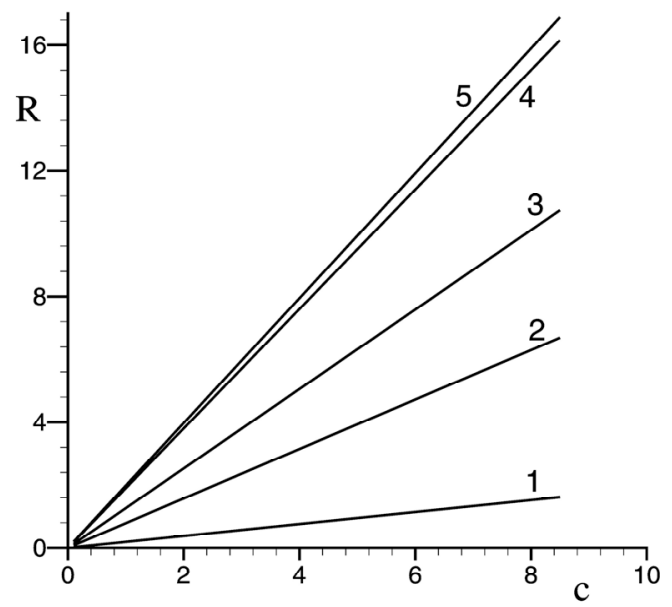

(b)

Fig. (32). A distribution function $\Phi\left(c_{x}, c_{z}\right)$ at $\in<10^{-2}$ and its characteristics, CEM-model, $h=0$. (a) - 3D representation of stationary DF $(t \rightarrow \infty)$. (b) - characteristics $R(c)$ at different times. Curve 1 refers to $t=0.1,2-t=0.5,3-t=1,4-t=3,5-t=5$. 


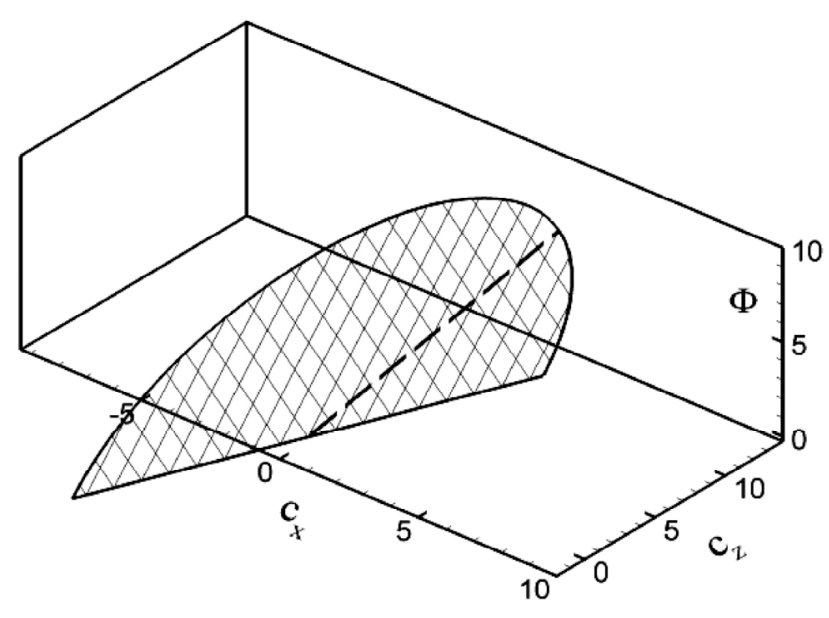

(a)

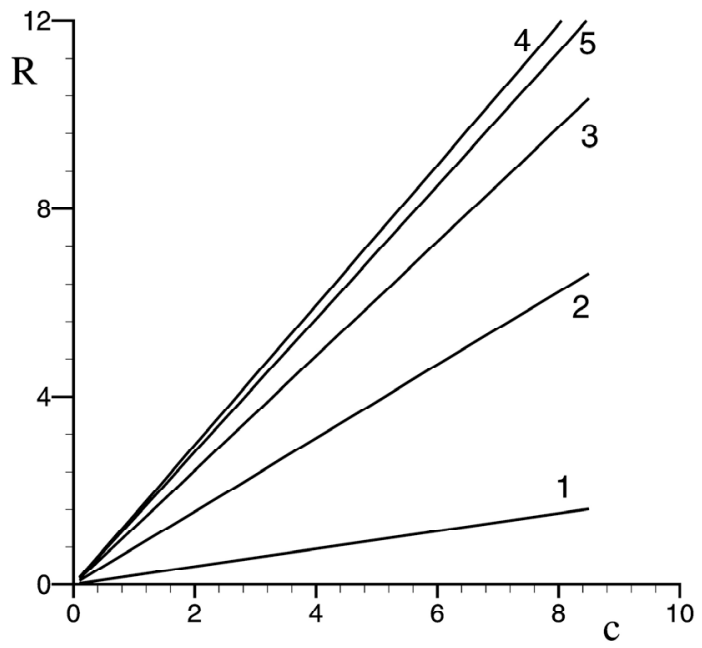

(b)

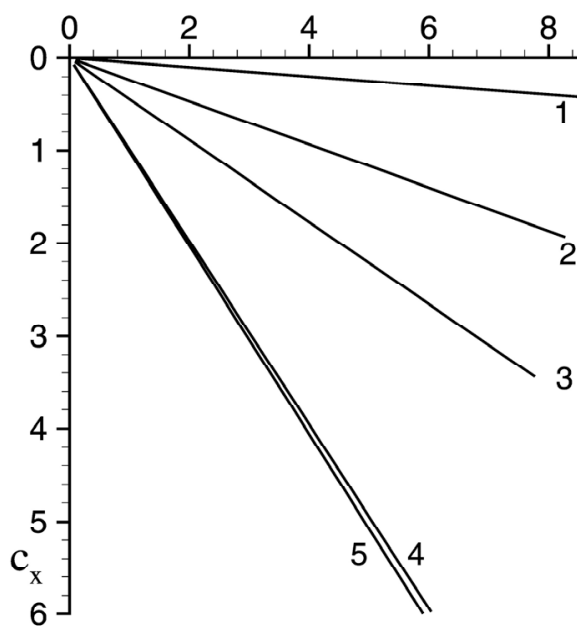

(c)

Fig. (33). Distribution function $\Phi\left(c_{x}, c_{z}\right)$ at $\in<10^{-2}$ and its characteristics, CEM-model, $h=1$. (a) - 3D representation of stationary DF $(t \rightarrow$ $\infty)$. (b) and (c) - characteristics of DF at different times. (b) $-R(c),(\mathbf{c})-$ projections $\alpha$-line on $c_{x}, c_{z}$-plane. Curve 1 refers to $t=0.1,2-t=$ $0.5,3-t=1,4-t=3,5-t=5$.

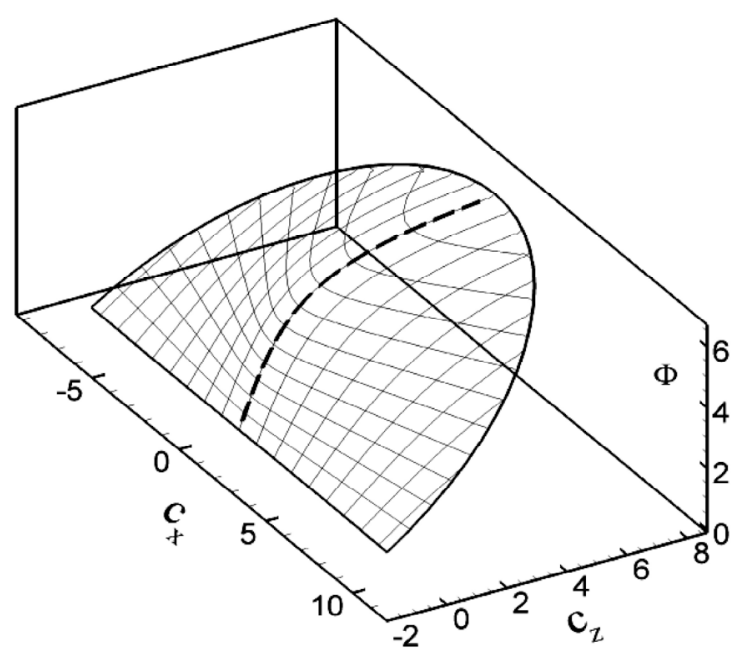

(a)

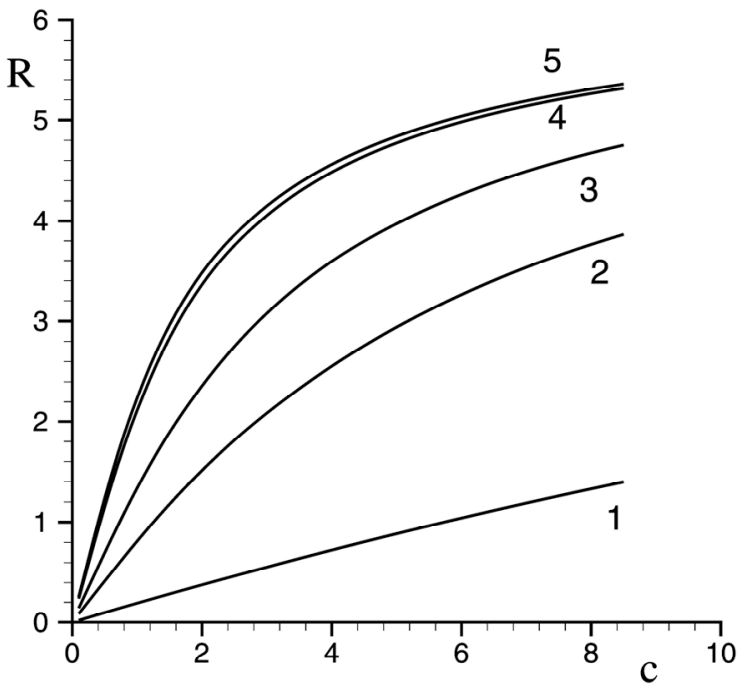

(b)

Fig. (34). Distribution function $\Phi\left(c_{x}, c_{z}\right)$ at $\in<10^{-2}$ and its characteristics, HS-model, $h=0$. (a) $-3 \mathrm{D}$ representation of stationary DF $(t \rightarrow$ $\infty)$. (b) - characteristics $R(c)$ at different times. Curve 1 refers to $t=0.1,2-t=0.5,3-t=1,4-t=3,5-t=5$. 


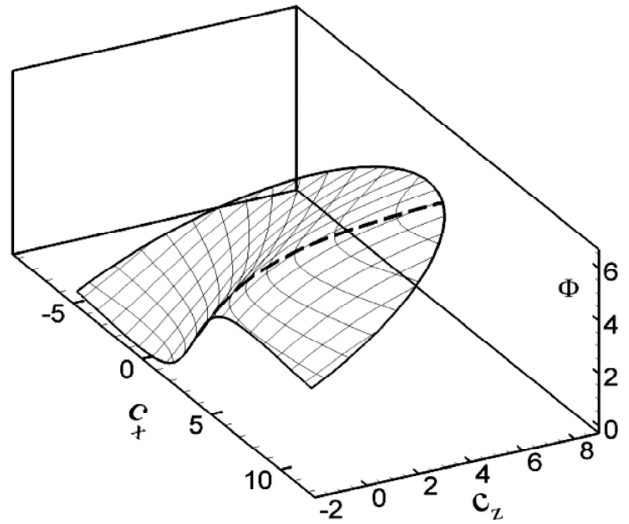

(a)

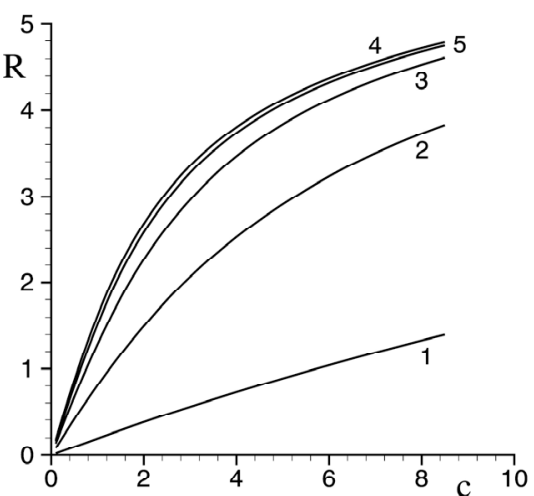

(b)

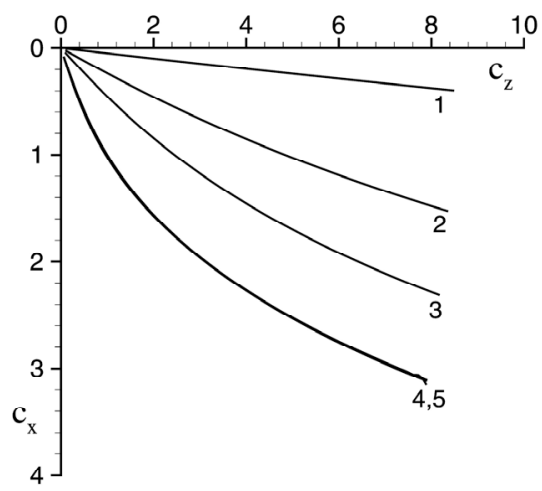

(c)

Fig. (35). Distribution function $\Phi\left(c_{x}, c_{z}\right)$ at $\in<10^{-2}$ and its characteristics, HS-model, $h=1$. (a) $-3 \mathrm{D}$ representation of stationary DF $(t \rightarrow$ $\infty)$. (b) and (c) - DF characteristics at different times. (b) $-R(c),(\mathbf{c})$ - projection of $\alpha$-line on $c_{x}, c_{z}$-plane. Curve 1 refers to $t=0.1,2-t=0.5$, $3-t=1,4-t=3,5-t=5$.

the direct ones. A limit angle, through which rotation occurs, increases with an increase in magnetic field. Note, that the evolution of the line of maximum proceeds nonmonotonously at $h \geq 1$. The oscillation of a line $R(c)$ being significantly more pronounced than that of $\alpha$-line.

As a third model we select the Coulomb interaction. In this case, a rotation of lines $R(c)$ and $\alpha(c)$ proceed more rapidly than in the previous cases. Especially fast is the rotation in a domain of high velocities. As a result, the line of maximums escape from a semi-plane $c_{z} \geq 0$ at $h \geq 0.5$. So, the calculation for the Coulomb interaction are presented for small values $h$ : 0, 0.1, 0.3 (Figs. (36-38)).

The calculation of DF $f(\mathbf{c})$ are carried out at moderate $(\epsilon$ $<1)$ and at strong $(\in \geq 1)$ electric fields. Here, there are also the lines of maximums in a domain of positive $c z$. Since, it is impossible to extract a small correction to the Maxwellian distribution now, and the $f(\mathbf{c})$ changes itself within many

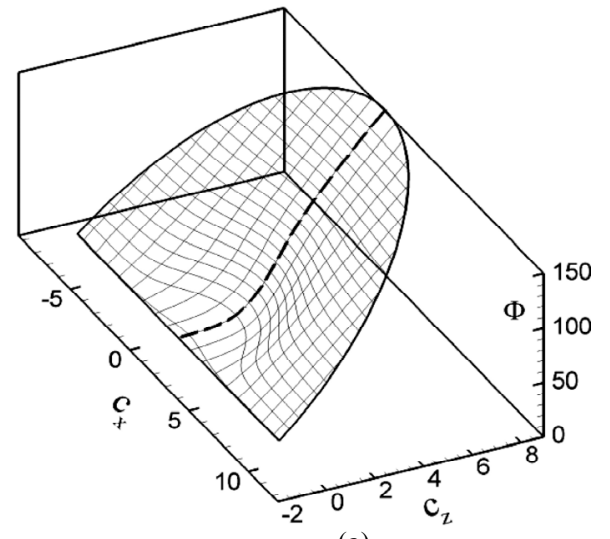

(a)

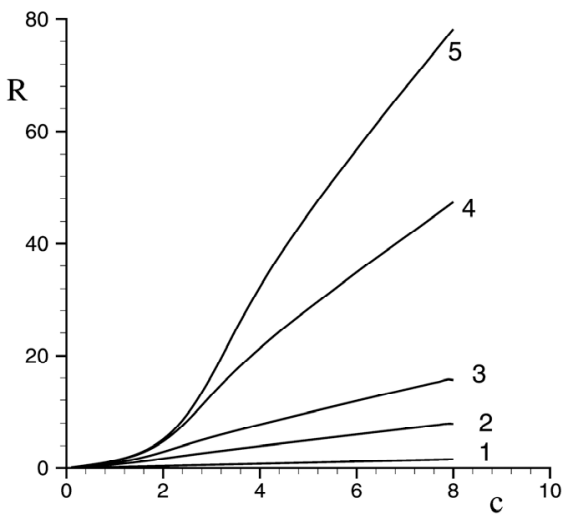

(b)

Fig. (36). Distribution function $\Phi\left(c_{x}, c_{z}\right)$ at $\in<10^{-2}$ and its characteristics, Coulomb interaction, $h=0$, (a) - 3D representation of stationary $\mathrm{DF}(t \rightarrow \infty)$. (b) - characteristics $R(c)$ at different times. Curve 1 refers to $t=0.1,2-t=0.5,3-t=1,4-t=3,5-t=5$. 


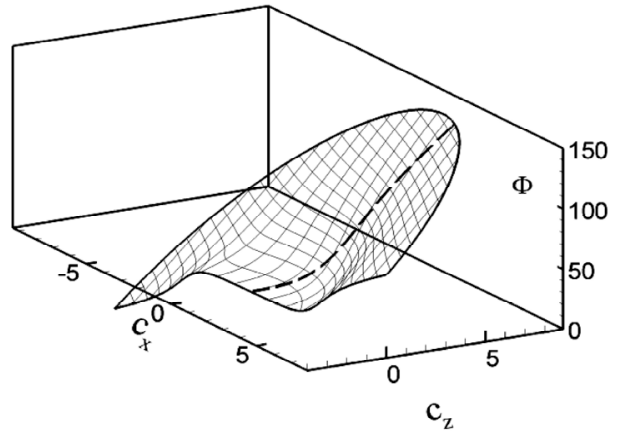

(a)

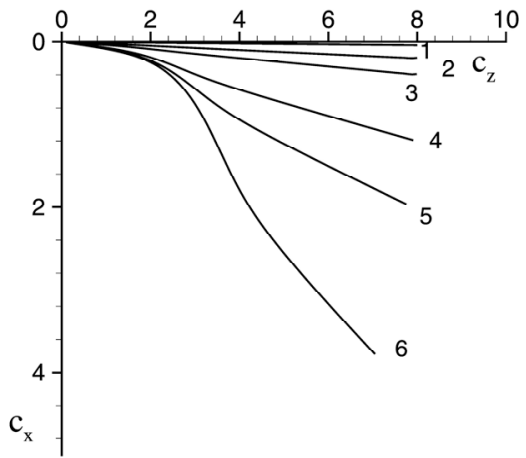

(b)

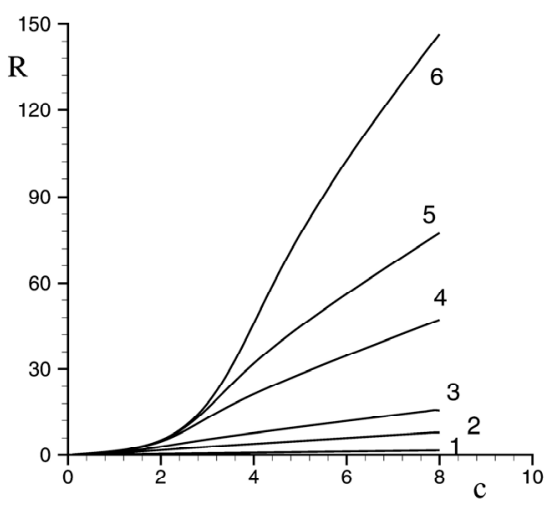

(c)

Fig. (37). Distribution function $\Phi\left(c_{x}, c_{z}\right)$ at $\in<10^{-2}$ and its characteristics, Coulomb interaction, $h=0.1$, (a) - 3D representation of stationary DF $(t \rightarrow \infty)$. (b) and (c) - DF characteristics at different times. (b) $-R(c),(\mathbf{c})-$ projections of $\alpha$-line on $c_{x}, c_{z}$-plane. Curve 1 refers to $t=0.1$, $2-t=0.5,3-t=1,4-t=3,5-t=5,6-t=10$.

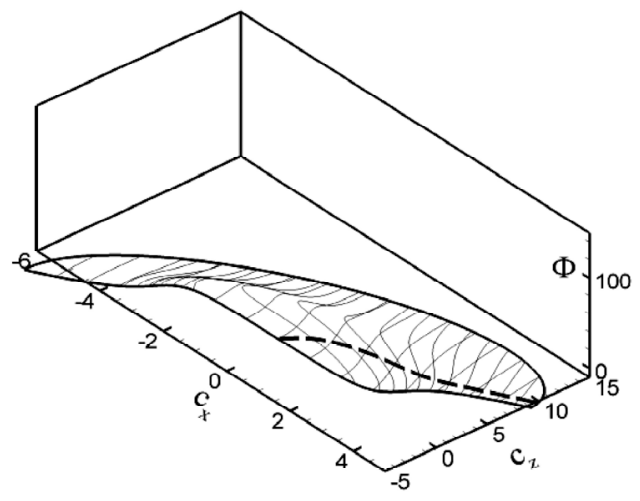

(a)

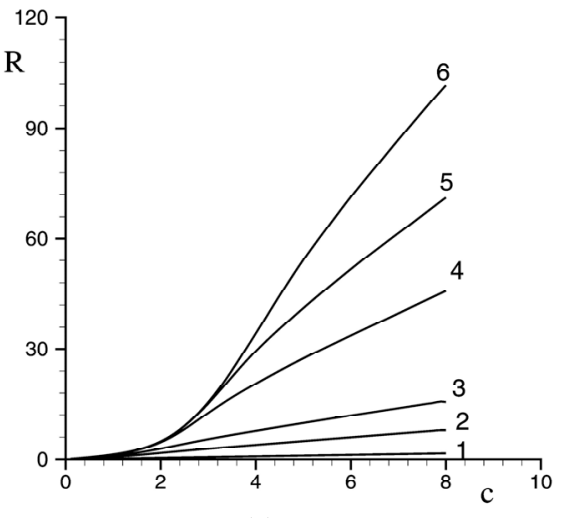

(b)

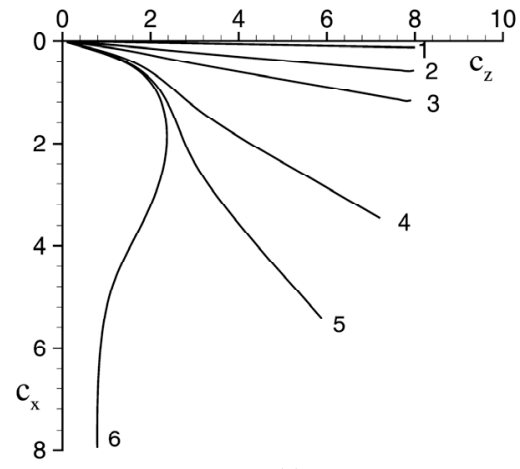

(c)

Fig. (38). Distribution function $\Phi\left(c_{x}, c_{z}\right)$ at $\in<10^{-2}$ and its characteristics, Coulomb interaction, $h=0.3$, (a) $-3 \mathrm{D}$ representation of stationary DF $(t \rightarrow \infty)$. (b) and (c) - DF characteristics at different times. (b) $-R(c),(\mathbf{c})-$ projections of $\alpha$-line on $c_{x}, c_{z}$-plane. Curve 1 refers to $t=0.1$, $2-t=0.5,3-t=1,4-t=3,5-t=5,6-t=10$.

orders of magnitude, 3D pictures do not show the features of DF behaviour. So, in $c y=0$-plane, the dependences $f(\psi)$ are built up at fixed values $c$. An example of such DF picture for hard-sphere model is shown in Fig. (39). 
f

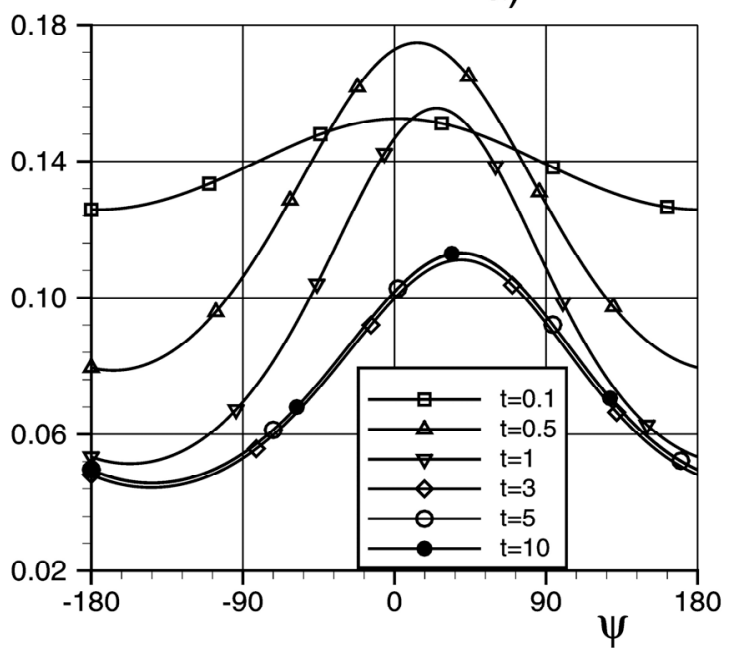

$f$

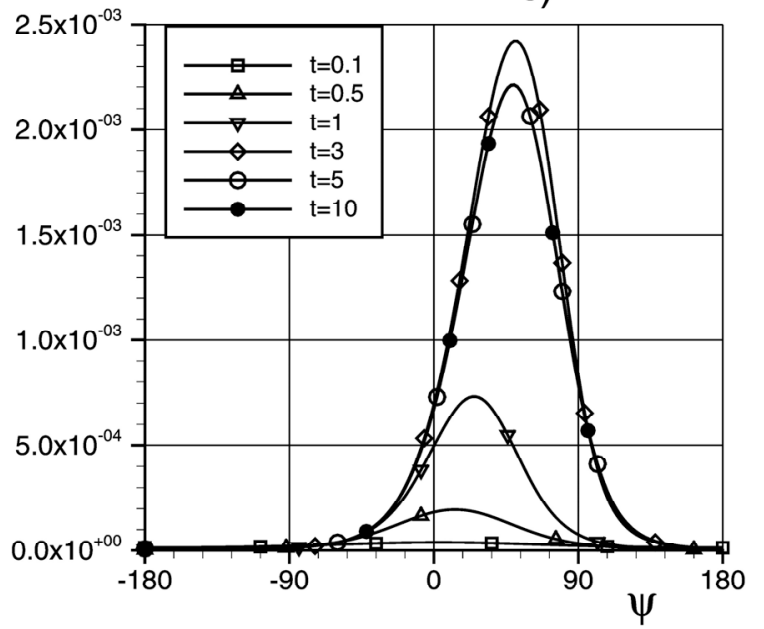

f

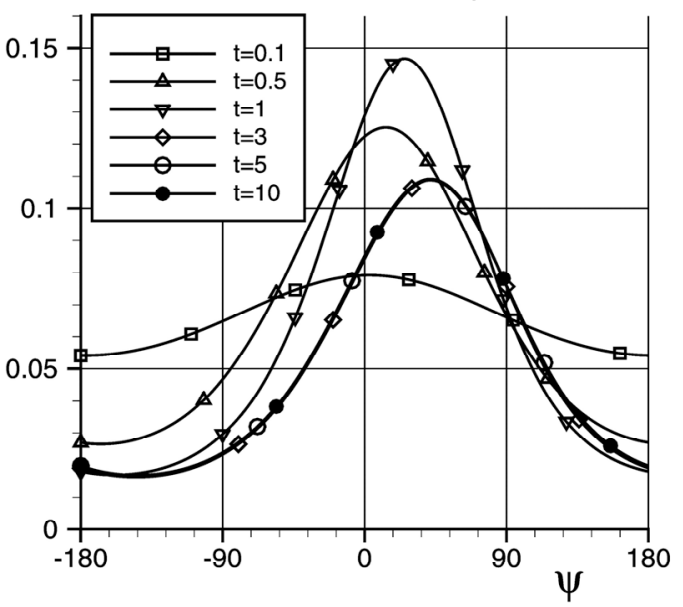

f

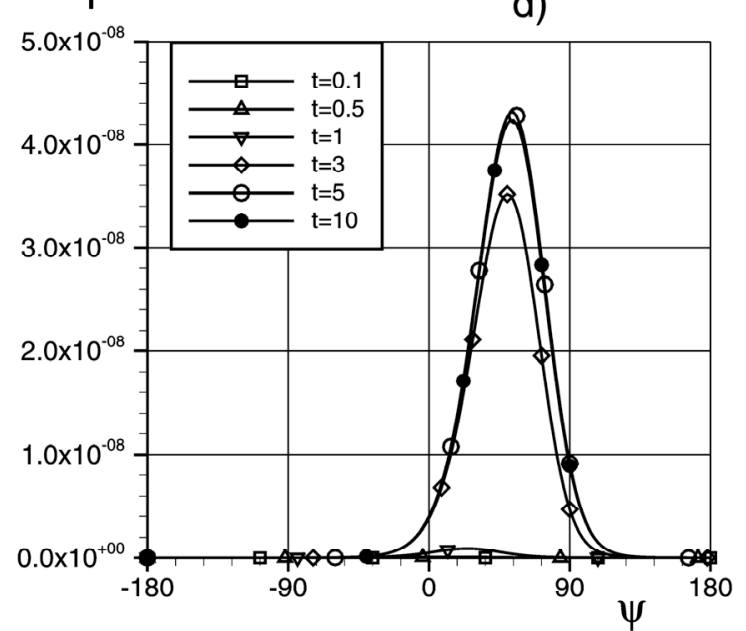

Fig. (39). Dependences $f(\psi)$ at different times with various values of velocity module $c$ for HS-model; $\in=1, h=1$ (a) refers to $c=0.5$; (b) $c=1 ;(\mathbf{c})-c=3 ;(\mathbf{d})-c=5$.

Similar figures are built up for other interaction models as well. In the case of Coulomb interaction a runaway process of ions is well seen at the weak magnetic field.

The Coulomb interaction is featured by the inherent slowly damped processes in a domain of high velocities under strong magnetic field. It is sufficient to compare the calculation results for different interaction models at $\epsilon=1$ and $h=10$ to see them, namely, Fig. (42) with Fig. (40) and Fig. (41). If (for CEM-and HS-models) the coordinates of peaks at $c=5$ converge to a limit value and an angle $\psi$ corresponding to maximum tends to $90^{\circ}$ the similar convergence is not observed in the case of Coulomb interaction; the strong oscillation of a peak proceeds up to $t$ $=10$. This process is of no less clarity at $h=5, h=3$ and $h=1$.

This phenomenon can be explained as follows. At initial stage of the proces, a portion of ions influenced by the strong electric field finds itself in a domain of high velocities. Further, their swarm moves practically without collision. In this domain, oscillation process is determined only by the electric and magnetic fields. Oscillation process in crossed $\epsilon$ and $h$ fields proceeds for a very long time without collisions.
A question arises concerning an eventual increase in density of this oscillating swarm when the magnetic fields are switched on with certain delay after the electric field involving the further progress in runaway process.

\section{CONCLUSION}

The moment method of the Boltzmann equation solution has a new impetus due to new results concerning the calculation of the matrix elements of large indices. The high possibilities of the moment method are shown while solving the kinetic problems that described the ion behaviour at the electric field and in crossed electric and magnetic fields. Not only the transport coefficients and physical moments are built up for the non-stationary process but the velocity distribution function as well. A number of features of DF behaviour are obtained, particularly in a domain of high velocities. Significantly curious are the quantitative results concerning runaway of ions in the case of Coulomb interaction as well as damping the effect at the strong magnetic field. Application limits and possible ways to overcome the restrictions of the standard moment method are found. 
$f$

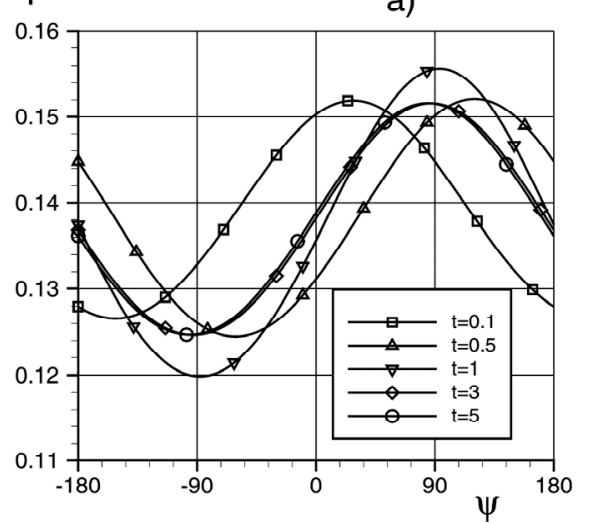

c)

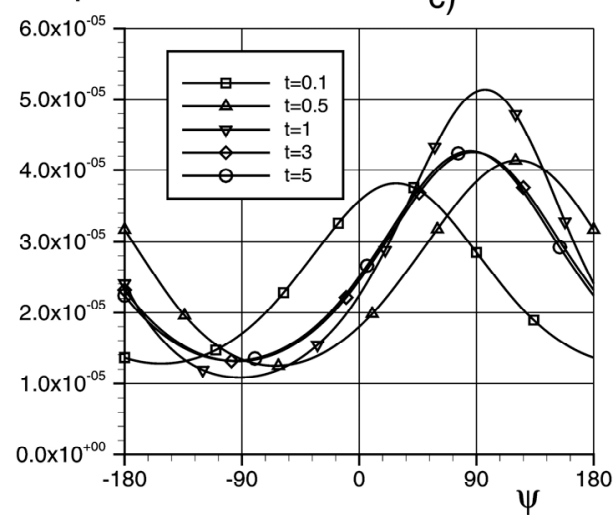

$f$

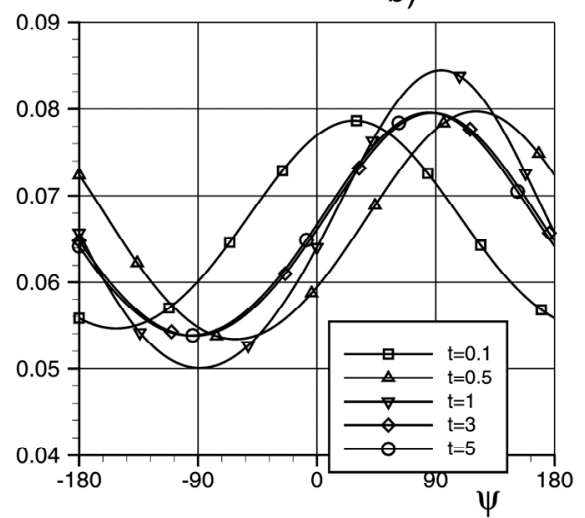

d)

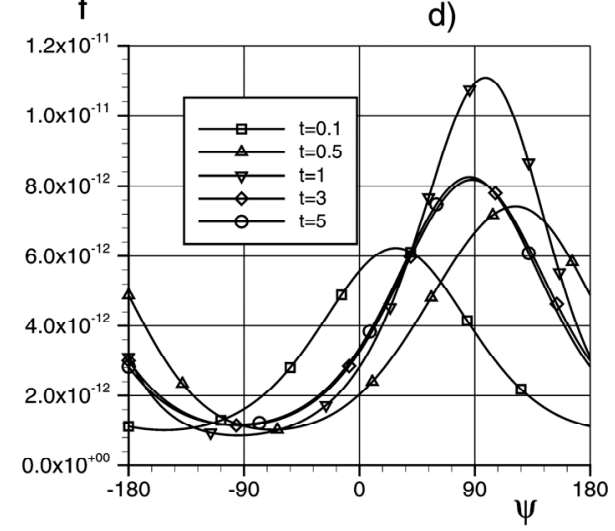

Fig. (40). Dependences $f(\psi)$ at different times with various values of velocity module $c$ for CEM-model; $\epsilon=1, h=10$ (a) refers to $c=0.5$; (b) $-c=1 ;(\mathbf{c})-c=3 ;(\mathbf{d})-c=5$.

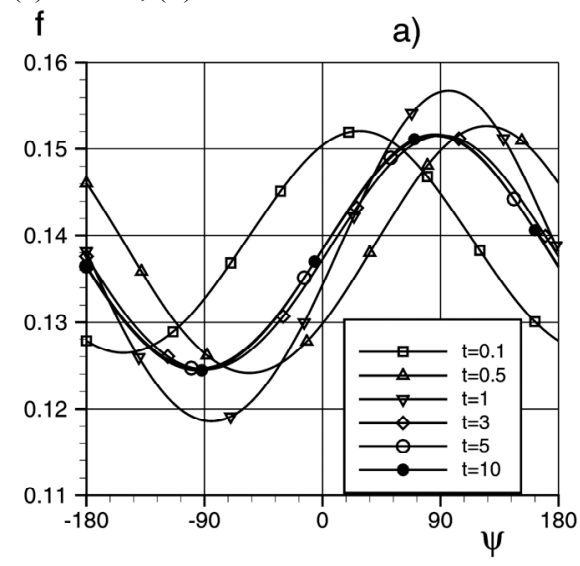

$f$ c)

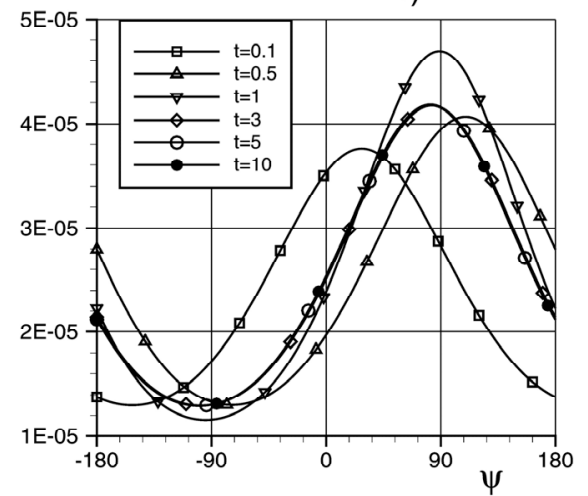

b)

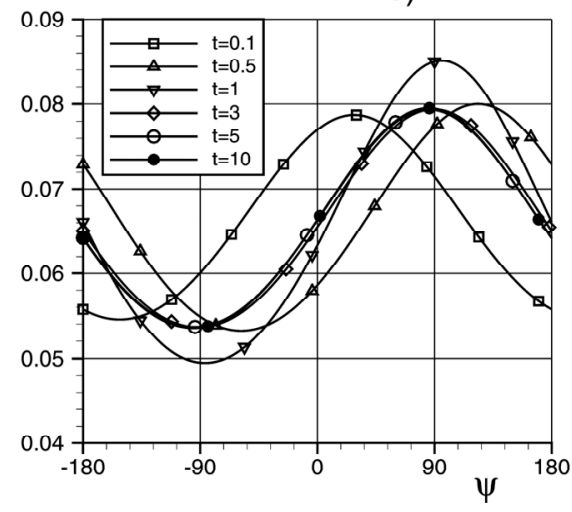

d)

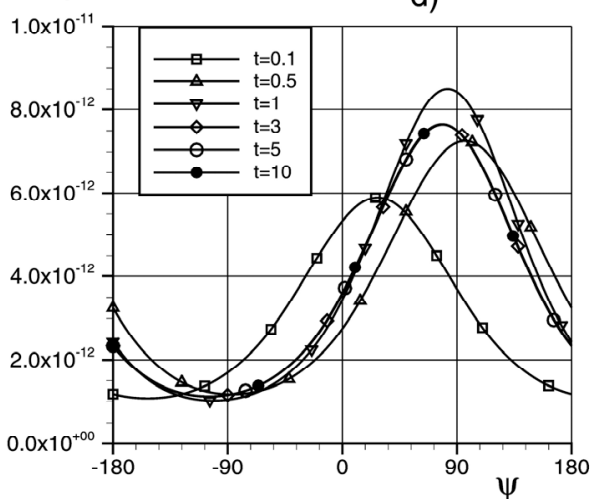

Fig. (41). Dependences $f(\psi)$ at different times with various values of velocity module $c$ for HS-model; $\epsilon=1, h=10$ (a) refers to $c=0.5$; (b) $-c=1 ;(\mathbf{c})-c=3 ;(\mathbf{d})-c=5$. 
f

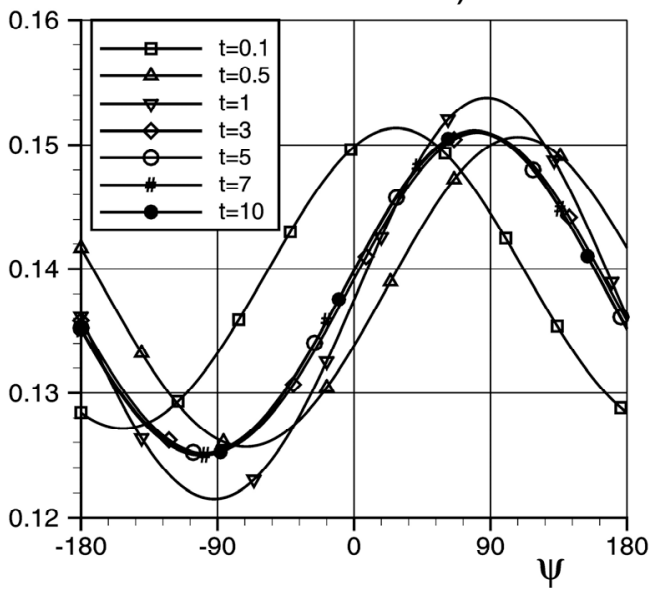

f

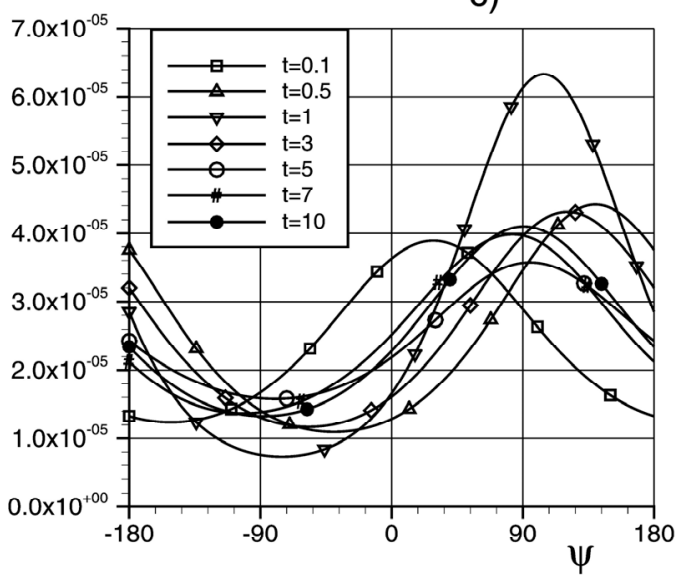

$f$

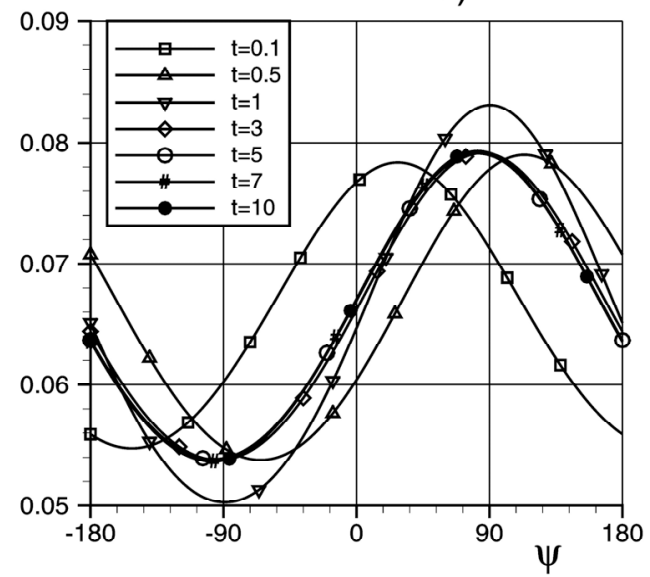

f

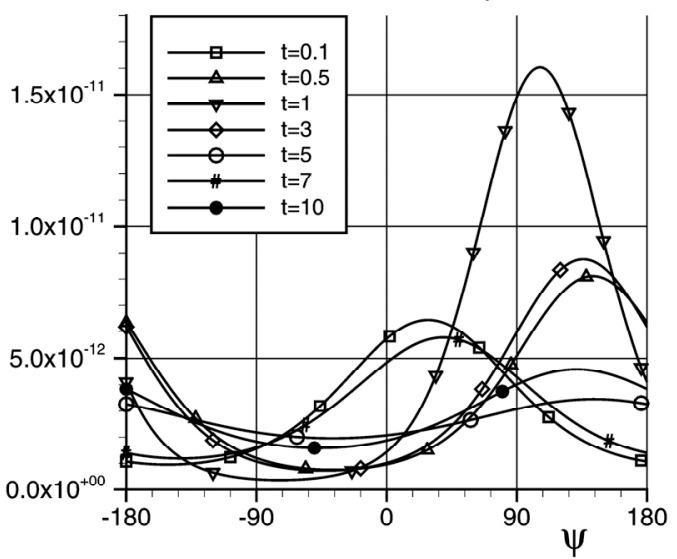

Fig. (42). Dependences $f(\psi)$ at different times with various values of velocity module $c$ for Coulomb interaction; $\in=1, h=10$ (a) refers to $c$ $=0.5 ;(\mathbf{b})-c=1 ;(\mathbf{c})-c=3 ;(\mathbf{d})-c=5$.

\section{ACKNOWLEDGEMENTS}

We are grateful to Dr. B. D'yakov for his aid in preparing this manuscript.

This work is supported by grants EOARD N FA8655-03D-0001/0017(CRDF Project N RUM1-1500-ST-04) and RFBR 06-08-01104.

\section{LIST OF SYMBOLS}
$E, \in$
$=$ Dimensional and dimensionless electric field strength
$H, h$
$=$ Dimensional and dimensionless magnetic field strength
$m_{i} \quad=$ Mass of ion, $m_{a}-$ mass of atom
$\lambda \quad=$ Mean free path
$\tau=$ Mean-time between collisions of an
$\sigma(g, \theta) \quad=$ Differential scattering cross-section
$K, K_{\text {hal }} \quad=$ Mobilities of ions
$T=$ Temperature of atoms
$n \quad=$ Concentration of ions
$v, c \quad=$ Dimensional and dimensionless velocity of ions
$f(\mathbf{v}), f(\mathbf{c}) \quad=$ Dimension and dimensionless

$M(c)$

I, $J$

$H_{j}, H_{r l m}^{i}$

$C_{j}, C_{r l m}^{i}$

$K_{j, k}^{i}, \Lambda_{r, r_{1}, l}^{(1)}, \Lambda_{r, r_{2}, l}^{(2)}$

$\varphi(c)$

$\mathbf{u}_{d}$

\section{REFERENCES}

[2] motion in a non-uniform gas. Proc Lond Math Soc 1935; 40: 382 435.

Ender AY, Ender IA. Polynomial expansions for the isotropic Boltzmann equation and invariance of the collision integral with respect to the choice of basis functions. Phys Fluids 1999; 11: 2720-30.

[3] Ender AY, Ender IA. Collision integral of the Boltzmann equation and the moment method. St.-Petersburg: St.-Petersburg University Press 2003; (in Russian). 
[4] Ender AY, Ender IA. Properties of the collision integral in the axisymmetric Boltzmann equation. Transp Theory Statist Phys 2007; 36(7): 563-88.

[5] Sena L. Motion of positive ions in electric field in plasma. ZETF 1946; 8: 734-8. (in Russian).

[6] Wannier GH. Motion of gaseous ions in strong electric fields. Bell Syst Tech J 1953; 32: 170-254.

[7] Perel VI. Calculation of the drift velocity of ions in the electric field in the gas. Sov Phys-JETP 1957; 5: 440-5.

[8] Smirnov VM. Ion mobility in their own gas. Zh Tekh Fiz 1966; 36: 1864-71. (in Russian).

[9] Mason EA, McDaniel EW. The mobility and diffusion in Gases. New York: Wiley 1973.

[10] Mason EA, McDaniel EW. Transport Properties of Ions in Gases. New York: Wiley 1988.

[11] White RD, Ness KF, Robson R.E. Development of swarm transport theory in radio-frequency electric and crossed electric and magnetic fields. Appl Surf Sci 2002; 192: 26-39.

[12] Li B, Robson RE, White RD. Magnetic field effects on spatial relaxation of swarm particles in the idealized steady-state Townsend experiment. Phys Rev E 2006; 74: 026405-1-13.

[13] Kumar K. Polynomial expansion in kinetic theory in gases. Ann Phys 1966; 37: 113-41.

[14] Kumar K, Skullerud HR, Robson RE. Kinetic theory of charge particle swarms in neutral gases. Aust J Phys 1980; 33: 343-406.

[15] Robson RE, Ness KF. Velocity distribution function and transport coefficients of electron swarms in gases: Spherical-harmonics decomposition of Boltzmann equation. Phys Rev A 1986; 33: 2068-77.

[16] Ness KF. Multi-term solution of Boltzmann equation for electron swarms in crossed electric and magnetic fields. J Phys D Appl Phys 1994; 27: 1848-61.

[17] Robson RE. Approximate formulas for ion and electron-transport coefficients in crossed electric and magnetic-fields. Aust J Phys 1994; 47: 279-301.

[18] Jovanovic JV, Vrhovac SB, Petrovic ZL. Momentum transfer theory of ion transport under the influence of resonant charge transfer collisions: The case of argon and neon ions in parent gases. Eur Phys J 2002; 21: 335-42.
[19] Hecke E. Über die Integralgleichung der kinetischen Gastheorie. Math Z 1922; 12: 274-86.

[20] Hilbert D. Begründung der kinetischen Gastheorie. Math Ann 1912; 72: 562-77.

[21] Chapman S, Cowling TG. The mathematical theory of non-uniform gases. Cambridge: Cambridge University Press 1939.

[22] Ferziger JH, Kaper HG. Kaper. Mathematical theory of Transport Processes in Gases. Amsterdam: North-Holland Publishing Company 1972.

[23] Ender I.A, Ender AY. On one of representation of the Boltzmann equation. Sov Phys Dokl 1971; 15: 633-6.

[24] Ender AY, Ender IA. System of moment equation in different bases. In: R.N.Miroshin, Ed. Aerodynamics, St.-Petersburg: NIICh of SPbSU 2003; pp. 179-203. (in Russian).

[25] Kihara T. The mathematical theory of electrical discharges in Gases. B. Velocity-distribution of positive ions in a static field. Rev Mod Phys 1953; 25: 844-52.

[26] Bhatnagar PL, Gross EP, Krook M. A model for collision processes in gases. Phys Rev 1954; 94: 511-5.

[27] Robson RE, Makabe T. Transport-coefficients and velocity distribution function of an ion swarm in an ac electric-field obtained from the bgk kinetic-equation. Aust J Phys 1994; 47: $305-$ 14.

[28] Sugawara H, Tagashira H, Sakay Y. The velocity distribution of ions in warm gas under a constant collision-frequency symmetric charge exchange model. J Phys D Appl Phys 1996; 29: 1168-74.

[29] Ender IA, Ender AY. Distribution function and transport properties of charged particles in electric field. 4th Polyakhov readings $\mathrm{SPb}$ : VVM Conference Proceedings 2006. 455-64. (in Russian).

[30] Tropp EA, Bakaleinikov LA, Ender AY, Ender IA. Asymptotics of collision integral matrix elements in the isotropic case. Tech Phys 2003; 48: 1090-102.

[31] Bakaleinikov LA, Ender AY, Ender IA. Calculation of the collision integral linear kernel for Maxwellian molecules in the isotropic case. Tech Phys 2006; 51: 1110-20.

[32] Tompson RV, Loyalka SK. Chapman-Enskog solution for diffusion: Pidduc's equation for arbitrary mass ratio. Phys Fluids 1987; 30: 2073-5.

\begin{tabular}{llr}
\hline Received: November 26, 2008 & Revised: February 16, 2009 & Accepted: February 17, 2009
\end{tabular}

(C) Ender et al.; Licensee Bentham Open.

This is an open access article licensed under the terms of the Creative Commons Attribution Non-Commercial License (http://creativecommons.org/licenses/by$\mathrm{nc} / 3.0 /$ ), which permits unrestricted, non-commercial use, distribution and reproduction in any medium, provided the work is properly cited. 